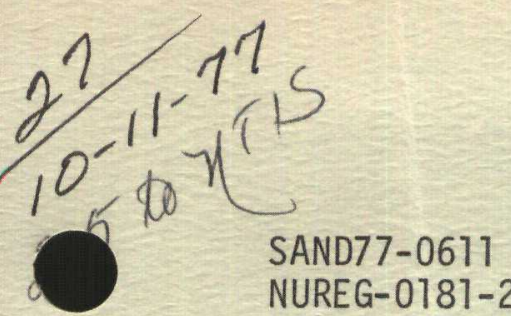

NUREG-0181-2

Unl imited Release
Distribution

Category NRC-7

\title{
FAST REACTOR SAFETY RESEARCH
}

PROGRAM QUARTERLY REPORT

JANUARY - MARCH 1977

Advanced Reactor Research Department

Prepared by Sandia Laboratories, Albuquerque, New Mexico 87115 and Livermore, California 94500

for the United States Nuclear Regulatory Commission under ERDA Contract AT(29-1)-789.

Printed August 1977

\section{Sandia Laboratories}

\section{Nuclear Fuel Cycle Programs}




\section{DISCLAIMER}

This report was prepared as an account of work sponsored by an agency of the United States Government. Neither the United States Government nor any agency Thereof, nor any of their employees, makes any warranty, express or implied, or assumes any legal liability or responsibility for the accuracy, completeness, or usefulness of any information, apparatus, product, or process disclosed, or represents that its use would not infringe privately owned rights. Reference herein to any specific commercial product, process, or service by trade name, trademark, manufacturer, or otherwise does not necessarily constitute or imply its endorsement, recommendation, or favoring by the United States Government or any agency thereof. The views and opinions of authors expressed herein do not necessarily state or reflect those of the United States Government or any agency thereof. 


\section{DISCLAIMER}

Portions of this document may be illegible in electronic image products. Images are produced from the best available original document. 
Issued by Sandia Laboratories, operated for the United States Energy Research \& Development Administration by Sandia Corporation.

\section{NOTICE}

This report was prepared as an account of work sponsored by the United States Government. Neither the United States nor the United States Energy Research and Development Administration, nor the United States Nuclear Regulatory Commission, nor any of their employees, nor any of their contractors, subcontractors, or their employees, makes any warranty, expressed or implied, or assumes any legal liability or responsibility for the accuracy, completeness or usefulness of any information, apparatus, product or process disclosed, or represents that its use would not infringe privately owned rights.

Printed in the United States of America Available from

National Technical Information Service

U. S. Department of Commerce

5285 Port Royal Road

Springfield, VA 22161

Price: Printed Copy $\$ 600$; Microfiche $\$ 3.00$ 


\section{MAJOR CONTRACTOR'S RECOMMENDATION FOR DISPOSITION OF SCIENTIFIC AND TECHNICAL DOCUMENT}

* See Instructions on Reverse

1. ERDA Report No. SAND 77-0611

2. Subject Category No. NUREG-0181-2

3. Title

Fast Reactor Safety Research Program Quarterly Report January - March 1977

Advanced Reactor Research Department

4. Type of Document (" $X$ " one)

a. Scientific and Technical Report

$\square$ b. Conference paper:

Title of conference

Date of conference

Exact location of conference

c. Other (Specify, Thesis, Translation, etc.)*

5. Copies Transmitted (" $X$ " one or more)

[1] a. Copies being transmitted for standard distribution by ERDA-TIC.

$\square$ b. Copies being transmitted for special distribution per attached complete address list.*

$\square$ c. Two completely legible, reproducible copies being transmitted to ERDA-TIC.

6. Recorproned Distribution (" $X$ " one)

a. Normal handling (after Patent clearance): no restraints on distribution except as may be required by the security classification.

$\square$ b. Make available only to U.S. Government agencies and their contractors.

$\square$ c. Make available only within ERDA and to ERDA contractors.

$\square$ d. Make available only within ERDA.

e. Make available only to those listed in item 12 below.

f. Other (Specify)*

7. Recongmended Announcement (" $X$ " one)

1 a. Normal procedure may be followed.*

$\square$ b. Recommend following announcement limitations:

8. Reason for Restrictions Recommended in 6 or 7 above.

$\square$ a. Preliminary information.

$\square$ b. Prepared primarily for internal use.

$\square$ c. Other (Explain)

9. Patent Clearance (" $X$ " one)

a. ERDA patent clearance has been granted by responsib le ERDA patent group.

$\square$ b. Document has been sent to responsible ERDA patent group for clearance.

10. National Security Information (For classified document only; " $X$ " one)

$\square$ a. Document does contain national security information other than restricted data.

b. Document does not contain national security information other than restricted data.

11. Copy Reproduction and Distribution

a. Total number of copies reproduced

447

b. Number of copies distributed outside originating organization

277

12. Additional Information or Remarks (Continue on separate sheet, if necessary)

13. Submitted by (Name and Position) (Please print or type)*

W. L. Garner - Supervisor, Division 3151

14. Organization

Sandia Laboratories

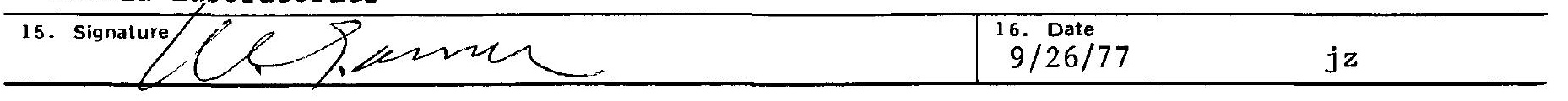




\section{INSTRUCTIONS}

Who uses this Form: All ERDA contractors except those specifically instructed by their ERDA contract administrator to use the shorter Form ERDA-427

When to Use: Submit one copy of this Form with each document which is sent to ERDA's Techncial Information Center (TIC) in accordance with the requirements of ERDA Manual Chapter 3201

Where to send. Forward this Form and the document(s) to

$$
\text { USERDA-TIC }
$$

P.O Box 62

Oak Ridge, TN 37830

\section{Item instructions:}

Item 1. The furst element in the number shall be an ERDAapproved code to be determined as follows (a) The responsible field office may request TIC approval of a unique code for a contractor, e $g$, BNL, BMI, HNL, etc, (b) A program division may request TIC approval of a unique code for a program or series of reports, e $\mathrm{g}$, PNE, VUF, etc, (c) An operations office may instruct a contractor to use the code approved for the operations office, $1 \mathrm{e}$, COO, ORO, IDO, SRO, SAN, ALO, RLO, NVO, and (d) Program divisions shall use the code ERDA for reports which they themselves prepare unless there is reason to use some other approved code

The code shall be followed by a sequential number, or by a contract number plus a sequential number, as follows (a) Contractors or programs with unique codes may complete the report number by adding a sequential number to the code, e g, HNL 101 , HNL-102, etc , or PNE-1, PNE-2, etc , or they may add the identifying portion of the contract number and a sequentral number, e g, ABC-2105-1, ABC-2105-2, etc, (b) Contractors using the operations office code shall complete the report number by adding the identifying portion of the contract number and a sequential number, $\mathrm{e} g$, COO-2200-1, COO-2200-2, etc , (c) Subcontractor reports shall be identified with the code used by the prime contractor, and (d) Program divisions using the ERDA code shall complete the report number by adding a sequential number which they request from the Library Branch, Division of Administrative Services

Item 2. Insert the appropriate subject category from TID-4500 ("Standard Distribution for Unclassified Scientific and Technical Reports") or M-3679 ("Standard Distribution for Classified Scientific and Technical Reports") for both classified and unclassified documents, whether or not printed for standard distribution

Item 3. Give title exactly as on the document itself unless title is classified In that case, omit title and state "classified title" in the space for item 3

Item 4. If box c is checked, indicate type of item being sent, e.g., thesis, translation, etc

Item 5. a If box a is checked, the number of copies specified for the appropriate category or categories in M-3679 or TID 4500 shall be forwarded to TIC for distribution.

b If box b is checked, complete address list must be provided TIC. c If box $\mathrm{c}$ is checked, at least one copy shall be original ribbon or off set and be completely legible A clear carbon copy is acceptable as a second reproducible copy

Item 6. If box a is checked for an unclassified document, it may be distributed by TIC (after patent clearance) to addressees listed in TID-4500 for the appropriate subject category, to libraries in the U.S and abroad, which through purchase of microfiche maintain collections of ERDA reports, and to the National Technical Information Service for sale to the public

If box a is checked for a classified document, it may be distributed by TIC to addressees listed in M-3679 for the appropriate subject category

If a box other than a is checked, the recommended limitation will be followed unless TIC receives other instructions from the responsible ERDA program division

Box f may be checked in order to specify special instructions, such as "Make avallable only as specifically approved by the program division," etc

Item 7. a. Announcement procedures are normally determined by the distribution that is to be given a document If box a in item 6 is checked for an unclassified document, it will normally be listed in the weekly "Accessions of Unlumited Distribution Reports by TIC" (TID-4401) and may be abstracted in "Nuclear Science Abstracts" (NSA)

A classified document, or an unclassified document for which box b, c, d, e, or $f$, in item 6 is checked, may be cited with appropriate subject index terms in "Abstracts of Limited Distribution Reports" (ALDR)

b. If the normal announcement procedures described in $7 \mathrm{a}$ are not appropriate check $7 \mathrm{~b}$ and indicate recommended announcement limitations

Item 8. If a box other than a is checked in item 6 , or if $7 \mathrm{~b}$ is checked, state reason for the recommended restriction, e g, "preliminary information," "prepared primarily for internal use," etc

Item 9. It is assumed that there is no objection to publication from the standpoint of the originating organization's patent interest Otherwise explain in Item 12

Item 10. If box a is checked, document cannot be made avalable to Access Permit holders (Code of Federal Regulations, 10 CFR, Part 25, subpart 25 6), if box $\mathrm{b}$ is checked, TIC will determine whether or not to make it avalable to them

Item 11. Self explanatory

Item 12. Use this space if necessary to expand on answers given above, e $g$, item $6 \mathrm{f}$ and item 8

Item 13. Enter name of person to whom inquiries concerning the recommendations on this Form may be addressed

Item 14-16. Self explanatory 
SAND77-0611

NRC-7

NUREG-0181-2

Unlimited Release

Printed August 1977

\author{
Fast Reactor Safety Research Program* \\ Quarterly Report \\ January - March 1977
}

Submitted by

Sandia Laboratories, $* \star$ Albuquerque, New Mexico 87115

Principal Organizations:

Reactor Studies Division, R. L. Coats, Supervisor

Radiation Physics Division, J. E. Powel1, Supervisor

Thermomechanical and Physical Research Division, B. M. Butcher, Supervisor

Reactor Safety Studies Division, D. A. Dahlgren, Supervisor

Design Technology Division, R. E. Nicke11, Supervisor

Environmental Research Division, B. D. Zak, Supervisor Reactor Theory and Analys is Division, W. S. Camp, Supervisor

APPROVED :
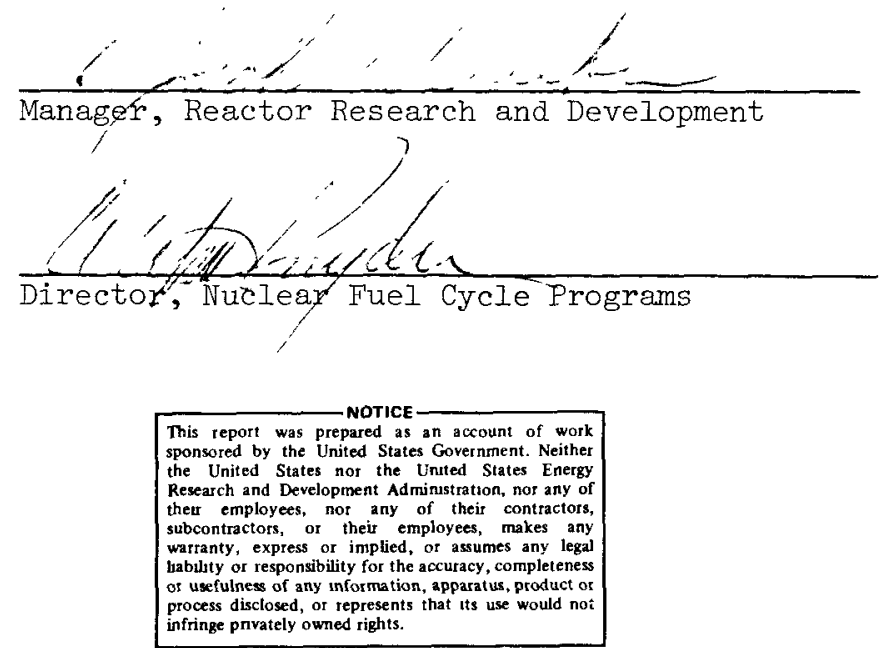

*This work was supported by the U. S. Nuclear Regulatory Commission (Project Nos. A-1016, A-1054, A-1048, A-1172, A-1181, and A-1186).

**Operated for the U. S. Energy Research and Development Administration by Sandia Laboratories under Contract AT(29-1)-789. 


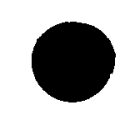

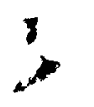

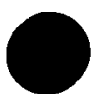


Introduction . . . . . . . . . . . . . . . . . . . . . . . 5

Executive Summary .. . . . . . . . . . . . . . . . . . . . . 11

1 Accident Energetics . . . . . . . . . . . . . . . . . . . . . . 27

1.1 Prompt Burst Excursion (PBE) Experiment . . . . . . . . . . . . . 27

1.2 Equation of State ......................... . . . . . . . . . . . . 42

1.3 Transition Phase Studies ..................... . . . . . 48

1.4 Aerosol Source Normalization ................... . . . . 51

1.5 Fuel Coolant Interactions . . . . . . . . . . . . . . . . . . . 52

1.6 Large-Scale Test Capabilities... . . . . . . . . . . . . . . . . . . 52

2 Core Debris Behavior . . . . . . . . . . . . . . . . . . . . 65

2.1 Molten Core Technology . . . . . . . . . . . . . . . . . . 65

2.2 Debris Bed Studies ........................... . . . . . . . . . . . . . . . . . . . . . . . . . . . . . . . . .

2.3 Molten Fuel Pool Studies . . . . . . . . . . . . . . . . . . 73

3 Sodium Containment and Structural Integrity . . . . . . . . . . . . . . 79

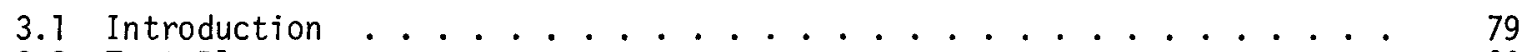

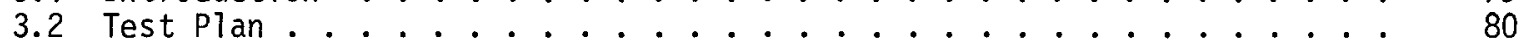

3.3 Concrete Crucibles . . . . . . . . . . . . . . . . . . . . . 80

3.4 Instrumentation . . . . . . . . . . . . . . . . . . . . . 85

3.5 Test Facility .. . . . . . . . . . . . . . . . . . . . . . . . . . . . . . . . . . . .

3.6 Gas and Posttest Chemical Analysis................. . . . . 89

3.7 CACECO Predictions . . . . . . . . . . . . . . . . . . . . . . . . 90

3.8 Acoustic Wave Measurements in Concrete . . . . . . . . . . . . . . . . 91

3.9 Cell Liner Stress Analysis . . . . . . . . . . . . . . . . . . . 92

4 Research for Elevated Temperature Design Criteria . . . . . . . . . . . . 93

4.1 General . . . . . . . . . . . . . . . . . . . . 9 93

4.2 Subtask on Creep-Fatigue . . . . . . . . . . . . . . . . . . . 93

4.3 Subtask on Cell Liners . . . . . . . . . . . . . . . . . . . . . . . . . . . . . . . . . . . . . . . . . . . . . . .

4.4 Subtask on Creep Buckling . . . . . . . . . . . . . . . . . . . . . . 96

4.5 Subtask on the Surveillance Test Loop... . . . . . . . . . . . . . 96

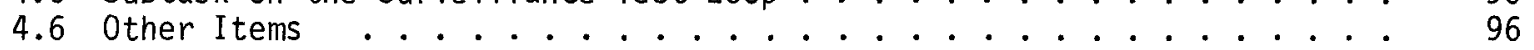

5 Fuel Motion Detection . . . . . . . . . . . . . . . . . . . 97

5.1 Introduction . . . . . . . . . . . . . . . . . . . . . 97

5.2 In-Core Fuel Motion Detection . . . . . . . . . . . . . . . . . . 97

5.3 Electron Beam Flash X-Radiography . . . . . . . . . . . . . . . . . . 104

5.4 Coded Aperture Imaging . . . . . . . . . . . . . . . . . . . . . . . . 108

6 ACPR Fuel Motion Detection System . . . . . . . . . . . . . . . 111

6.1 Introduction ......................... . . 111

6.2 SPR II Fuel Pin Imaging Tests . . . . . . . . . . . . . . . . . . . . 111

6.3 Fission Track Fuel Motion Monitor System . . . . . . . . . . . . . . 135

References 142 
Sodium and Piston Velocity and Pressure . . . . . . . . . . .

Top Pressure Transducer . . . . . . . . . . . . . . . . 30

Bottom Pressure Transducer . . . . . . . . . . . . . . . . . . .

Top Pressure Transducer for Heat Transfer Case . . . . . . . . .

Bottom Pressure Transducer for Heat Transfer Case . . . . . . . . .

Pressure History at the Top of the PBE-7S Experiment . . . . . . .

Pressure History at the Bottom of the PBE-7S Experiment . . . . . .

Radiograph of the PBE-7S Experiment . . . . . . . . . . . . . . . .

Particle Size Distribution from the PBE-3E and $4 \mathrm{~S}$ Experiments

and the $\mathrm{S} 3$ and $\mathrm{E} 2$ Experiments . . . . . . . . . . . . . . .

Pressure and Energy Histories from EEOS-U02-4 . . . . . . . . . . .

Comparison of EEOS Results with Other Experimental Data . . . . .

Reactivity Time Histories for Fuel Motion and Sodium Void . . . . . Infinite Medium Parametric Study of BeO-UO Fuels for Gas-Cooled Systems--25\% Coolant, 65.9\% Fuel. . . . . . . . . . . . . . Externally Pulsed System . . . . . . . . . . . . . . . . . Spatial Flux versus Time for Pulse Source. . . . . . . . . . . . Top Hat Assembly . . . . . . . . . . . . . . . . . . . . . . . . . Small Capsule Experiment Package . . . . . . . . . . . . . . . . Slump and Water/Cement Ratio for CRBRP Concrete Pours . . . . . . 90-Day Strength and Water/Cement Ratio for CRBRP Concrete Pours . . Prototypic Insulated Liner . . . . . . . . . . . . . . . . . Location of Instrumentation . . . . . . . . . . . . . . . . Thin Film Thermocouple Junction . . . . . . . . . . . . . . . Eight Junction Thermocouple . . . . . . . . . . . . . . . . . Hermes II Coded Source Flash X-Ray Chamber Proposal (side view) . Hermes II Coded Source Flash X-Ray Chamber Proposal (top view) . Configuration for the Fuel Pin Imaging Experiment at SPR II . . . Laser Reconstruction of the Fuel Pin Recorded on X-Ray Film . . . . Laser Reconstruction of the Fuel Pins Recorded with

the X-Ray Image Intensifier . . . . . . . . . . . . . . . . . . .

Enriched Pin at SPR II . . . . . . . . . . . . . . . . . . .

Scintillator Geometry and Laser Light Scattering Modes . . . . . .

Layered Scintillator Construction . . . . . . . . . . . . . . . .

Schematic of the Collimation Test Module . . . . . . . . . . .

Geometry for Signal-to-Background Ratio Estimate . . . . . . . . .

Geometry for Estimate of $\gamma$-Ray Albedo and Transmitted Flux

from a Lead Layer . . . . . . . . . . . . . . . . . . . . .

Fuel Rod Motion Detector Assembly . • . . . . . . . . . . . . . . .

Log Display of Spectrum of Alpha Particles Transmitted through a Sample with a Track Density of $9.8 \times 10^{2} \mathrm{~cm}$ in the Foreground, and through a Sample with $1.1 \times 10^{6}$ Craters $/ \mathrm{cm}^{2}$ in the Background.. . 
FAST REACTOR SAFETY RESEARCH PROGRAM

QUARTERLY REPORT

\section{INTRODUCTION}

Sandia Laboratories Fast Reactor Safety Research Program, initiated in FY 1975, 1-9 is a comprehensive research activity conducted on behalf of the U. S. Nuclear Regulatory Commission (NRC) and is part of NRC's confirmatory research effort to assure that the necessary safety data and theoretical understanding exist to adequately license and regulate the Liquid Metal Fast Breeder Reactor (LMFBR) or other advanced convertors and breeders which may be commercialized in the United States. The program includes a broad range of experiments to simulate accidental transient conditions in the LMFBR to provide an understanding of the important processes which determine the adequacy of current and proposed safety systems and to assure that other unexpected phenomena do not occur. Such a program must also include the development of analytical models, verified by experiment, which can be used to predict reactor performance under a broad variety of abnormal conditions. This work, along with that of other U. S. and international researchers, should provide the technology base on which licensing decisions can be made in confidence that the safety of the pubtic is assured.

The early thrust of Sandia's program was designed to provide data associated with the hypothetical core disruptive accident, with emphasis on prompt burst ( $\sim$-ms period) energetics and the behavior of post-accident core debris. The scope of the program was expanded in FY's 1976 and 1977 to encompass other inherent retention concerns such as large-scale sodium containment and structural integrity, aerosol source studies, transition phase energetics, fuel failure and motion, and studies to quantify elevated temperature failure modes of critical component materials. A portion of the present effort in the program is directed toward obtaining data to support the licensing review of the $\mathrm{Cl}$ inch River Breeder Reactor (CRBR). Another important early thrust of the program is the development of the test facilities and techniques. For FY 1977, the program is organized in the following subtasks, progress on which is reported herein.

TASK 1 Accident Energetics

Subtask 1.1 - Prompt Burst Energetics - Studies performed on single- and multi-pin geometries (oxides, carbides, and nitrides) with and without sodium to determine the consequences of fuel failure under conditions of prompt burst power excursions. The ACPR is operated in the pulse mode with initial periods as short as $1.3 \mathrm{~ms}$. 
Subtask 1.2 - Equation-of-State (EOS) - The equation-of-state for hightemperature vapor pressure for fresh and irradiated oxide fuels and advanced fuels will be obtained. Sandia's electron beam and reactor facilities will be used for transient heating of reactor fuels to high temperatures $(3,000$ to $10,000 \mathrm{~K})$ and pressures ( 1.5 to $300 \mathrm{MPa}$ ) to obtain the required EOS data.

Subtask 1.3 - Iransition Phase - Work in this task will be carried out to better define and resolve key uncertainties in the transition phase of LMFBR accident sequences. There will be two main areas of investigation: (a) support and experimental testing of SIMMER and other relevant transition phase computer codes and (b) a study of key phenomenological problems relevant to the transition phase. The latter work need not necessarily be directed toward the support of a specific computer code.

Subtask 1.4 - Aerosol Source Normalization - Aerosol source normalization determined from in-pile experiments will characterize physical properties of fuel particles resulting from the breakup of fuel pins subjected to simulated overpower excursions in the ACPR. The characteristics of these particles will be compared with those produced by out-ofpile techniques at ORNL. Subsequent in-pile studies will examine the interaction of particles with the supporting structure and with sodium.

Subtask 1.5 - Fuel-Coolant Interactions - Fuel-coolant interaction studies will be conducted to determine the potential for the rapid transfer to energy from the fuel to the coolant. Initial efforts are concentrated on simulation tests (see Subtask 1.1). As results from these tests are obtained, phenomenological studies will be developed as appropriate. Emphas is in the future will be on the advanced fuels.

Subtask 1.6 - Large-Scale Test Capabilities - (a) Full-Length Capabilities (HFFPR). A facility capable of performing large phenomenological experiments at short periods is under study. Such a facility would provide a considerable extension of capability beyond the upgraded ACPR and would provide the basis of NRC's phenomenological test program in the 1980's. In addition to LMFBR safety tests, the facility (due to the large area access to the core) would provide excellent capability for development of reactor safety diagnostics, particularly material motion detection systems, and for performance of experiments which will observe the motion of core materials in simulated accidents. (b) Large-Scale Tests. One of the main problems 
facing the U. S. LMFBR Safety Program is a lack of adequate facilities to do large-scale testing. New facilities and upgrades of existing facilities have been proposed, but there is a possibility that they will not be available in time to support a 1986 commercialization decision. The Nation's Underground Nuclear Test Technology Program, which utilizes the facilities at the Nevada Test Site, has the potential for providing some of these needed data from 5 to 7 years before the proposed safety test facilities will be on-line. Studies are currently underway to determine the feasibility of such tests. Included in the study are source-driven, self-driven, and hybrid tests.

\section{TASK 2 Core Debris Behavior}

Subtask 2.1 - Molten Core Technology = Studies will be performed in the areas of molten $\mathrm{UO}_{2}$ and steel interactions with steel liners and concrete for application to inherent retention concepts. The capability will be developed to include application to other core retention materials. Analys is of the accident sequences will be included in this task.

Subtask 2.2 - PAHR Debris Bed - Post-accident heat removal (PAHR) debris bed studies will be undertaken to characterize the thermal behavior of internaliy heated debris beds to determine their melt-through potential in order to provide information needed for analys is of post-accident containment. The experiments will utilize fission heating and will be performed in-pile using the ACPR (Annular Core Pulse Reactor) in the steady-state mode.

Subtask 2.3 - PAHR Molten Pool - PAHR molten pool studies will be conducted to characterize the thermal and kinetic behavior of internally heated molten pools of fuel materials, including fission product retention, and to determine the melt-through potential of such configurations to be used in analysis of post-accident containment. The experimental program will utilize fission heating of $\mathrm{UO}_{2}$ fuel and will be performed in-core using the ACPR in the steady-state mode.

TASK 3 Sodium Containment and Structural Integrity

This experimental study of large-scale sodium interactions with reactor materials is in support of the NRC confirmatory research program for sodium-cooled reactor systems. The immediate effort will be divided into two parts: sodium/concrete interactions and sodium/liner interactions. The sodium/concrete interactions 
focus on the exothermic reactions occurring when sodium is brought into rapid contact with concrete, with emphasis on the following four objectives:

(a) quantitative measurement of the quantity and composition of the evolved gases; (b) quantitative measurement of the penetration rate of the sodium reaction zone into the concrete, including estimates of thermal cracking and exfoliation; (c) identification of the influence of special engineering features such as the presence of cracks and steel reinforcements on the sodium penetration rate; and (d) development or confirmation of an analytic model of the process for use in 7 icensing verification. These objectives will be supplemented by thermochemical measurements as appropriate. Generic Southeastern U. S. and other concrete types with possible LMFBR applications will be investigated. The liner interaction will be an analytic and experimental program to explore the consequences of hot sodium spills or sprays on steel liners over concrete substructures. This task will specifically address the situation in which a flaw in either one of the liners permits sodium to breach the liner and come in contact with the concrete substructure. The objective of this task is to establish the conditions for which the sodium/concrete reactions are sufficient to cause further damage to the liner and limit its effectiveness.

TASK 4 Research of Elevated Temperature Design Criteria

The primary objectives of the elevated temperature design criteria studies are: (a) to develop correlative NDE techniques that can be used to monitor progressive creep, fatigue, and combined creep-fatigue damage in LMFBR component materials; (b) to study the parameters that induce failure in steel liners following an accidental sodium spill in an equipment cell; (c) to evaluate the analytical methods and correlate with experimental data for creep buckling of LMFBR components operating at elevated temperatures; and (d) to design, fabricate, and operate a multiaxial creep-fatigue facility for the purpose of evaluation of creep-fatigue damage rutes.

TASK 5 Fuel Motion Diagnostics

Subtask 5.1 - In-Core Systems - Facilities such as SLSF and EBR II do not have slots for high-resolution fuel motion detection devices. On large tests (subassembly and multi-assembly), planned for SAREF and other facilities, self-shielding may preclude using techniques such as coded aperture imaging, flash x-radiography, and the neutron hodoscope. The feasibility of in-core fuel motion detection will be investigated through analysis and experiments. The development of unfolding techniques and miniature radiation detectors, as well as experiments in SPR III utilizing a 37-pin bundle, are part of this task. 


\section{Subtask 5.2 - Electron Beam Flash X-Radiography - Electron beam flash x-radiography will be investigated as a possible high-resolution, two-dimensional fuel motion detection device for up to subassembly size experiments. Point source as well as coded source techniques will be studied. Source requirements, resolution, detection systems, and ultimate system costs will be considered. \\ Subtask 5.3 - Coded Aperture Imaging - During FY 1975 and FY 1976, Sandia performed studies to support the definition of a fuel motion detec- tion system for the ACPR Upgrade. A system was defined and is now being developed under the program (see Task 6 ). These studies have the goal of defining an envelope of capability for full-7ength LMFBR fuel pins and to support system selection for SAREF. The high-resolution, three-dimensional capabilities of the technique will be emphasized.}

\section{TASK 6 ACPR Fuel Motion System}

A fuel motion detection system is to be develpped for the ACPR Upgrade. The design of the system will be based upon the experience gained in experiments with shielding, collimation, coded aperture imaging, and time-resolved pseudoholography at the ACPR, SPR II, and ${ }^{252}$ Cf facilities. The system will be designed for high spatial and temporal resolution measurements of fuel motion in both transient and steady-state experiments, including 1- and 7-pin prompt burst excursion experiments, bed leveling and material separation studies in the postaccident heat removal experiments.

\section{TASK 7 ACPR Performance Improvement of the Annular Core Pulsed Reactor}

This task is reported in a separate quarterly report -- see Annular Core Pulse Reactor Upgrade Quarterly Report January-March 1977, SAND77-0610, Sandia Laboratories, Albuquerque, NM, June 1977. 
-

• 
EXECUTIVE SUMMARY

Accident Energetics

\section{Prompt Burst Energetics}

A major concern associated with a hypothetical core-disruptive accident (HCDA) is the ability of the pressure vessel to survive the conditions produced within, by the accident. The potential exists for severe pressure conditions due to evaporization of the fuel and clad and possibly due to rapid heat transfer to more efficient working fluids such as the coolant.

The Sandia program in prompt burst energetics is aimed at (1) identifying the phenomena which dominate in the conversion of thermal energy to work, developing models to accurately predict the energetics associated with such hypothetical accidents; and (2) providing a data base to validate these models in the prompt burst regime $(\sim 1 \mathrm{~ms}$ period, a time scale where no data existed prior to this activity. This program includes the examination of the integral effects of fuel-clad-coolant interactions, fission gas release, and fuel and fission product vapor pressures during superprompt critical core-disruptive conditions. The experimental work is closely interfaced with analytical efforts to develop models which uniquely describe the observed phenomena for incorporation into predictive accident analys is used in the regulatory process. These experiments also provide information about the states and distribution of fuel, clad, and coolant following a superprompt excursion. These data serve as initial conditions for post-accident heat removal and inherent retention studies. To date, the experiments have concentrated on the response of intact fuel pins to prompt critical excursions such as might exist in low power regions of a core undergoing a loss-of-fluid (LOF) accident. Future experiments will refine the conditions for simulating this portion of the scenario as well as addressing the behavior of disrupted materials subjected to prompt critical excursions (PBE).

In tests performed to date, single fresh $\mathrm{UO}_{2}$ fuel pins have been pulse fission heated in the Sandia Annular Core Pulsed Reactor (ACPR) to temperatures resulting in fuel vaporization. The pins, which have been surrounded by helium (simulating pin in voided low-power region of core) or sodium, are contained in a rigid pressure vessel which is instrumented with thermocouples and pressure transducers and is fitted with a movable 
piston which also acts as an inertial head at its upper end. Measurements of the conversion of thermal energy to work result from comparisons of the kinetic energy of the piston to the fission energy input to the fuel. Pressure, temperature, and piston displacement histories are determined for a variety of fuel-coolant systems and initial conditions. A series of experiments with fresh uranium dioxide fuel and sodium coolant is in progress. A series with uranium carbide and sodium will be performed later this year. Current modeling efforts involve use of EXPAND, a Sandia developed in-clad fuel motion and clad failure code, to model the excursion up to the point of clad failure. The multiphase lagrangian hydrocode, CSQII, which we had previously employed to model postfailure conditions, modeled fuel-coolant interactions as a Hicks-Menzies-like situation. or ignored them completely. We are now using the Argonne developed code EPIC to model post-failure conditions. This code employs a simple Cho-Wright model of fuel-coolant interactions. It is our intention to improve this aspect of EPIC. In addition, EPIC, as received, could not model correctly the dynamics of fuel vaporization. The cause of this problem has been identified, and the code will be modified accordingly.

During this reporting period, the PBE-7S experiment was performed and involved a fresh $20 \%$ enriched $\mathrm{UO}_{2}$ fuel pin in sodium subjected to a single maximum pulse in the ACPR. This experiment was a repeat of PBE-6S. The results, inciuding short duration, large pressure pulses (5400 psi), and a small thermal energy to work conversion efficiency $\left(\sim 10^{-4}\right)$, were similar to previous experiments. Gross pin failure (as indicated by large pressure events) occurred earlier in the ACPR pulse during PBE-7S than during PBE-6S, resulting in lower measured pressures. The hydrodynamic modeling was extended to include motion of the piston, yielding analytical results in qualitative agreement with the experimental results of PBE-5S.

In a1l experiments, significant in-clad axial fuel motion has been present in a time frame corresponding to that time before clad rupture. To date, there has been no clear indication that an energetic fuel-coolant interaction has contributed to the work or high pressures observed. Although the measured pressures appear to be inconsistent with that expected from fuel vapor alone (utilizing the ANL equation-of-state formulation), it has not been shown conclusively that fuel vapor alone cannot account for the observed pressure or work. Near future experiments will utilize smaller $\mathrm{Na} /$ fuel ratios, higher initial $\mathrm{Na}$ temperatures, and more prototypic temperature profiles in the fuel region. This activity will hopefully permit elimination of an energetic fuel coolant interaction as a significant consideration in evaluation of HDCA energetics as well as define the fraction of the energy transferred from the fuel to the coolant in a time scale short enough to lead to significant pressure increases. 


\section{Equation of State}

One of the principal potential contributors to conversion of thermal energy to work during an HDCA is the vaporization of the core materials. In order for NRC to assess the adequacy of containment in regard to public safety, it is essential that the timedependent relationship between the vapor pressure of the individual core materials and their specific energy be known. This information is required both for predicting the energy released during a hypothetical excursion and for determining the amount of energy available to do damage to the pressure vessel and, hence, pose a threat to the containment system. The primary material which is of importance in this analysis is the fuel. Since the data base at these high temperatures of interest is inadequate, Sandia is currently developing these data. Plans are first to develop a consistent data set and theory for pure $\mathrm{UO}_{2}$ and then to provide similar data on fuels with fission products as well as advanced fuel types. Vapor pressure measurements of fresh $\mathrm{UO}_{2}$ over the temperature range of interest have now been completed, utilizing an electron beam technique and two types of ACPR reactor experiments. These three different techniques produce vapor pressure results which essentially superimpose on each other. The data also imply that significantly higher vapor pressures are achieved for a given energy level than predicted by formulations which are based on extrapolations from lower temperature steady-state experiments.

Since these new data are obtained over a time scale more characteristic of a prompt critical excursion than are the equilibrium data, they should be more appropriate for use in accident analys is codes. This difference may be due to rate effects in materials properties, and such effects are currently under theoretical investigation. The significance of these differences, in the determination of the total work potential during an HCDA, is currentiy a matter of some controversy and must be resolved. (To this end, a program of sensitivity studies, in which the vapor phase EOS and heat capacity used in VENUS are varied, will be instituted.) Nevertheless the importance of these results lies in the fact that they constitute a consistent body of information extending far beyond previously available equilibrium results. We thus have at hand methods for obtaining reliable EOS data for all oxide, carbide, and nitride fuels throughout the energy range of interest and over the correct experimental time scales for accident analys is.

\section{Transition Phase}

During the course of a hypothetical accident in a fast reactor, there may occur a disruption and redistribution of significant quantities of core materials. In such an event, the redistribution may result in a rapid increase in reactivity, placing the entire 
system in a prompt critical state. The resulting power excursion could lead to the rapid vaporization of core materials which, in turn, would do mechanical work on the pressure vessel as well as those components, such as the fuel region, within the containment.

Transition phase research is directed at the study of the disruptive core in order to determine if it is possible for a recriticality to occur due to the redistribution of core materials. The data and understanding generated in this study will provide a basis for NRC to assess the adequacy of given reactor containment systems. At the transition stage of a loss-of-flow accident, one or a number of subassemblies have melted down, but a prompt critical excursion resulting in mechanical disassembly has not taken place. The disruption could propagate radially as subassemblies are melted through. The progression of the transition phase is a key factor in determining the magnitude of an accident, and hence the energetics to be contained. This is because there is a potential for significant fuel removal at an early stage, and for relatively benign accident termination. At the present, however, this potential has not been demonstrated and fundamental aspects of transition phase phenomenology have not been resolved.

Initially two key questions need to be answered: (1) To what extend can the moltenfuel/fission gas mixture be dispersed in the early stages of core disruption. That is, how far can the mixture move axially before fuel freezing occurs and a blockage is formed.

(2) Assuming that blockages do exist and that the core is therefore bottled up, what is the behavior of the boiling pool of molten fuel and steel vapor. In particular, is steel vapor an adequate mechanism for maintaining a boiled-up and, hence, subcritical pool.

Clearly, if substantial early dispersal can be successfully demonstrated, then the second question above need not be addressed.

In the area of blockage formation, controversy exists as to the distance which molten fuel can penetrate axially into the upper subassembly. This uncertainty is related to the question of the stability of fuel crusts on molten steel. This is important because a crust acts as an insulator against convection, reducing the heat transfer between the molten fuel and the steel. If a crust does not exist, or is removed rapidly, then short penetration lengths wi11 occur.

Neither the condition under which crusts are stable nor the actual mechanism for crust removal is well-known. Experiments to study this are being considered with simulants under various boundary conditions. Along with the experiments, phenomenological modeling will be done. This work will make a contribution to improving existing transition phase computer codes. In addition, modeling will support the design and development of a large, nineteenpin, freezing experiment using $\mathrm{UO}_{2}-\mathrm{ZrO}_{2}$ thermite on pure $\mathrm{UO}_{2}$. This test will provide 
valuable data for identifying the most important physical processes involved in the phenomena as well as provide data for verification of computer codes, particularly SIMMER.

Out-of-pile boiling pool studies will begin shortly to study variations in void distribution and pool height as a function of power. These quantities are directly related to the question of criticality in a fuel/steel pool. Conceptual planning for an in-core, fissionheated boiling pool test has also been initiated.

\section{Aerosol Source Normalization}

In order to determine the potential threat to public safety in the event of a CDA, it is essential for NRC to consider the hypothetical case wherein the containment is breached during a hypothesized accident. Such a study requires detailed knowledge of the character of the radioactive material which could escape and be transported to the public, Such material in order to escape must pass through the core debris and the coolant, and must avoid plate-out on structural materials.

In order to accurately quantify such escape, it is essential to know precisely the form of the aerosols produced by other accidents.

The Sandia program is aimed at determining accurately the character of aerosols produced in extreme overpower excursions, and it will provide a means to normalize the aerosols being produced in the out-of-pile studies at ORNL. Succeeding work will study the interaction of the debris from these fission heated fuels with the containing structure and with the coolant. The particles generated in this study will be produced from fissionable material heated by neutrons in the ACPR.

A fairly complete description of this fuel breakup is obtained from the number of particles produced from the fuel, the size and shape of these particles, their initial velocities, and their production, each as a function of time. These characteristics are measured by collecting representative particles in channels designed into a rotating wheel. The collection surfaces covering the channels can be removed from direct viewing of the particles in a transmission electron microscope. By counting and sizing the particles as a function of their position in a channel, the desired characteristics unfold. These experimental data are compared with code results which, in turn, can predict conditions at nearby regions of interest. The experimental data can also be used as an initial particle source term for aerosol codes which predict the evoluation of these particles or aerosols as they interact with their environment inside and outside the reactor. Many studies have 
examined the behavior of particles produced by non-neutronic methods. A comparison of particles produced in this study with those produced by out-of-reactor techniques will begin to establish limits of interest on the particle densities and size spectra of debris from reactor fuels.

During this quarter the design and detailed drawings for the three subsystems of the experiment have been completed. The subsystems are: (1) The experimental canister housing the $\mathrm{UO}_{2}$ fuel element, and sample wheel, motor and speed monitoring transducer, a 1 amp and mirrors for illuminating and photographing the fuel, a thermocouple vacuum gage for monitoring the canister pressure, electrical connections to the fuel element to measure its resistance by a current-voltage measurement, and a thermocouple to measure fuel temperature before the experiment begins. (2) The optical train for photographing the fuel breakup event, consisting of several mirrors which carry the image outside the reactor over an optical path of about 10 meters for fast framing photography. (3) A passive pumping system which reduces the pressure in the experimental canister to $10^{-3}$ torr where the large mean free path of air molecules allows the smallest debris particles to travel to the sampling whee $2 \mathrm{~cm}$ away. All parts for the sybsystems are in fabrication and commercial orders are being placed for the first test in late summer.

\section{Fuel Dynamics Experiments}

Following the initiating phase of an accident, redistribution of core materials is an important process which can have a dominant effect on the probability of a subsequent energetic excursion. Of paramount importance in determining how fuel material may redistribute is the manner in which the fuel initially disrupts.

The current Sandia fuel dynamics activity is designed to determine the mode of fuel disruption as a function of hypothetical accident conditions and to develop a model which accurately predicts this behavior. These results will be used to improve the current accident analys is codes and to assess the safety of given reactor designs.

The current activity in this area is designed to investigate three aspects of fuel disruption in a loss-of-flow accident. These are:

(1) mode of disruption;

(2) time of onset of fission-gas driven fuel dispersal;

(3) rate of fission-gas driven fuel dispersat.

Initially a series of nine tests will be conducted in the ACPR reactor in which single pellets of irradiated fuel (about 5\% burnup) will be heated to melt in order to study the failure process. The test pellets will have microstructures and gas content characteristics of fuel irradiated at local power levels of 4 to $12 \mathrm{~kW} / \mathrm{ft}$. The disruption process will be viewed with high-speed motion picture cameras. 
This visual observation will produce the first direct evidence of the mode of fuel disruption. Previous evidence has been based only on indirect data such as hodoscope results and postmortem examinations. Recent results from the FI and L5 tests produced evidence that swelling of solid fuel and frothing of liquid fuel may be the dominant disruption modes. This is in direct contradiction to the so-called "dust-cloud breakup" theory which is the basis for modeling of fuel disruption in the SAS accident analys is code. Since the mode of fuel breakup can affect the dynamics and rate of subsequent fuel slumping or dispersal, direct observation of fuel disruption in these tests will substantially improve our ability to calculate reactivity effects of early fuel motion.

The time of onset and the rate of fission-gas driven fuel dispersal will be accurately measured in these tests. These are important parameters for advanced reactors which have substantial sodium void coefficients. This is because the outcome of a high-reactivity initiating phase can be very sensitive to small reactivity shifts. If fuel disperses immediately upon melting and at a rapid rate, effects such as a prompt critical excursion caused by FCI-driven sodium voiding (LOF-driven-TOP) may be avoided.

Models of fission gas bubble growth, coalescence, and buoyant motion in liquid fuel are being developed, and will be implemented for analysis of pellet frothing experiments. Later versions will include solid-state bubble dynamics and may explicitiy consider the possibility of solid-state fuel breakup.

\section{Material Motion Phenomena}

The direction of motion of core materials (fuel, cladding, sodium) during a fast reactor hypothetical accident can determine the severity of the event. Detailed studies of material motion, particularly fuel motion, during safety tests are therefore extremely important. As part of Sandia's experimental program for NRC, research is being conducted to study fuel motion phenomena (fuel breakup, LOF, TOP, etc.) under various reactor accident conditions. Near future tests will be conducted in the upgraded ACPR. Future tests will require larger bundle sizes and full-length fuel pins, and (depending upon the program direction) will be conducted in facilities such as SAREF, CABRI, SCARABEE, or special purpose facilities designed to address specific issues.

The development of the capability for measuring material motion during a test is a necessary first step in the program. Studies conducted at Sandia have indicated major limitations exist in current diagnostics systems -- particularly for large-scale tests where no proven capability exists. We have under development a high-resolution diagnostics system for the upgraded ACPR that will, if successful, provide a unique high-resolution 
capability for one- and seven-pin tests. Systems that would provide capabilities for larger scale tests are under study and feasibility should be established before FY 1980 .

\section{ACPR Upgrade Fuel Motion Detection System}

The purpose of this task is to develop a high-resolution fuel motion detection system for the ACPR upgrade. If the development is successful, the system will provide energetics, molten core, and fuel dynamics experiments currently underway in ACPR with critically needed time-resolved images of moving fuel. The technique being employed is coded aperture imaging in which radiation from the test fuel pins is modulated with a coded aperture to form pseudoholograms. The pseudoholograms are later reconstructed to form threedimensional images of the moving fuel with millimeter spatial and sub-millisecond temporal resolution. Critical dates in the program are: final design completion, February 1, 1978; positioning and shielding hardware installation, March 1, 1978; construction completion, June 1, 1978; final installation, August 1, 1978; and initial system testing completion, September 30, 1978.

During this quarter, a major milestone was reached in the program. A fuel pin irradiated with the SPR-II reactor was imaged with good contrast using the prototype system. Conditions (other than background) were similar to those expected in ACPR. Actual background conditions will be measured in experiments in ACPR planned for the next quarter.

\section{Coded Aperture Imaging}

This task is designed to study the applicability to other safety test facilities of the coded aperture imaging fuel motion detection technology being developed for the upgraded ACPR. To this end, problems associated with imaging full-length, greater than 7 -pin bundles have been identified and studied since the start of FY 77 . During this quarter, under direction from NRC, work was diverted from this task to a temporary assignment. In this assignment, a proposed set of baseline diagnostic requirements was determined for the ERDA/NRC test program to be conducted on SAREF. They will be submitted to NRC during the next quarter for inclusion at the next meeting of the steering group on SAREF diagnostics. The work on coded aperture imaging will resume during the next quarter.

\section{In-Core Fuel Motion Detection}

The development of in-core fuel motion detection techniques is important since some facilities to be used for NRC tests do not have instrumentation slots and self-shielding 
may preclude the use of high-resolution techniques on large-scale tests. The feasibility of an in-core diagnostic method is being investigated at Sandia. Experiments are being conducted on Sandia SPR-III to test out both detector and data unfold techniques.

A fuel positioning system with precise reactivity control and safety interlocks is required for these experiments. The design of this device was completed during this quarter. Miniature neutron detectors are also required and have been under development as a part of this task. Thin film fission couples that can fit inside the wire wrap of a test assembly show promise and will be used in the SPR-III experiments. Prototype devices have been fabricated this quarter and are currently being tests.

The key to success of the in-core approach is the capability of unfolding fuel movement from detector response data. This capability requires a transport theory formulation relating adjoint $\mathrm{flux}$, fuel density, and detector response. In the last quarter this formulation was derived. During the past quarter a computer code was written to calculate response kernels that appear in the formulation and relate detector response and fuel density distribution functions.

Work will continue in all of the above areas during the next quarter.

\section{Electron Beam Flash X-Radiography}

The feasibility of utilizing flash x-radiography for fuel motion measurements is being investigated. This technique offers the possibility of high temporal and spatial resolution in test sizes ranging up to a full subassembly with potential discrimination between fuel, clad, and sodium. These potential advantages, as well as questions about cost, impact on the test reactor core (a full through slot is required to pass the bremsstrahlung), multiple scattering distortion of images, and problems associated with imaging large (up to $2 \mathrm{~m}$ in length) objects are being studied.

Both conventional point source radiography and distributed source techniques, where resolution is recovered using coded aperture imaging principles (designated "coded source radiography"), are being considered. Experiments utilizing the large Sandia accelerator, Hermes II, have been in progress since the start of FY 77 . Currently preparations are being made for an experiment to study the coded source technique. During this quarter, a new coded source convertor has been designed and is under construction. Significant improvements in contrast are expected. A linear cathode optimized for coded source radiography is being constructed for Hermes II. 
Experiments on the multiple scattering problem were conducted using the Sandia Linatron facility. Data taken over an energy range of 5-10 MeV indicate a decrease in resolution degradation with increasing energy. High energy data will be taken in the near future to verify these conclusions. The coded source Hermes II experiment should be underway next quarter.

\section{Core Debris Behavior}

\section{Molten Core Technology}

Hypothetical core disruptive accidents of advanced reactor systems often lead to the presence of substantial amounts of molten fuel and steel. Sandia's Molten Core Technology task focuses on the development of a technology to produce and handle prototypic, hightemperature, large-scale melts to develop a data base and phenomenological understanding of the interaction of molten core debris with containment materials. Currentiy, the associated experiments involve the material interactions of prototypic melts with the components of the inherent retention concept, other retention concepts, and stee 1 representative of liners and vessels.

A number of thermitically generated steel melts were contacted with steel 1 iners. The results indicate a penetration of the liner with a time scale of about a second. This tends to support arguments stating that molten pools will rapidly escape the reactor vessel and that cavity liners will not be intact if molten pools are developed.

The facility and experimental technique development has continued at a good pace during this reporting period. Discussions have been held with contractors for integrating the furnace electrics and hot-zone design. The results appear optimistic. The experimental design for the thermite/concrete experiments has been proof tested, and the top hat for the sustained steel/concrete tests is in fabrication.

A comprehensive concrete model has been developed which utilizes the basic data produced in the thermal decomposition experiments.

\section{Debris Bed Post-Accident Heat Removal}

During some time period following a hypothetical core-disruptive accident, there is postulated to exist layers of solid $\mathrm{UO}_{2}$ particulate debris on horizontal surfaces either within the reactor vessel or external to it. This debris, formed by the rapid cooling and fragmentation of molten $\mathrm{UO}_{2}$ when it contacts liquid sodium, is continually heated by the 
decay of the fission fragments it contains. Should the cooling afforded by surrounding $\mathrm{Na}$ be insufficient, the temperature will continue to rise until the melting temperatures of the $\mathrm{UO}_{2}$ or of adjacent restraining materials are reached, and the possibility of a release to the public is increased. In order for NRC to establish the criteria for acceptance for containment designs and to assess those designs, it is essential to determine the limits of decay power and bed configuration beyond which the overhead coolant can no longer effectively remove the heat.

To date all debris bed studies have utilized simulant materials or improper heating of real materials. (In order to establish the correct thermal behavior, it is necessary that heat be generated primarily in the $\mathrm{UO}_{2}$ of the $\mathrm{UO}_{2}-\mathrm{Na}$ mixture.) The Sandia in-pile debris bed experiments represent the first experiments in which proper heating conditions will be established with real materials, and they will afford the first unambiguous bench mark data on debris bed coolability. Tests will be conducted with debris beds having various characteristics and various internal heat generation levels to define coolability and dryout conditions. This activity uses liquid sodium and urania which are heated by fissions in a nuclear test reactor to simulate the debris bed decay heat. Because experiments of this kind are complicated and use potentially hazardous materials and processes, a large amount of planning and testing is required before they can be executed.

During this reporting period, the major effort was the testing of the apparatus to be used during the reactor tests. In the out-of-pile systems tests (OPST), the principal objective is to verify the thermal performance of the debris bed experiment package and the supporting helium cooling loop. During the tests, a $10 \mathrm{~kW}$ electrical heater, immersed in liquid sodium, simulated the debris bed. The test was performed at the 25-foot deep test hole at the outdoor test station. The system is performing well, and testing is continuing, with the first reactor test to be conducted next quarter.

\section{Molten Pool Post-Accident Heat Removal}

In order to evaluate further post-accident heat removal possibilities, it is necessary to consider the case in which the debris formed by the hypothesized accident cannot be cooled and progress to a molten state. In such a case, it is essential to determine the characteristics and rate of melting attack on the containment barriers in order to determine if the barrier will be penetrated and, if so, in what time frame.

Depending on the particular accident scenario, pools can be formed (1) in the lower region of the core (as a result of fuel melting and slumping), (2) on horizontal surfaces within the reactor vessel but outside the core region (as a result of remelting solid 
debris or directly due to massive penetration of core supports and streaming), or (3) in regions external to the reactor vessel (assuming the reactor vessel has been penetrated).

In all cases, heating is supplied to fuel intrinsically. In case (1), fission as well as fission product decay contribute to the fuel heating. In cases (2) and (3), the principal heat source is fission product decay. It is important to understand the behavior of such pools in order to determine if a pool of given characteristics will be retained within engineered barriers and, if not, what retention times are afforded by these barriers to permit reduction of decay heat levels.

The in-pile molten pool experiments are aimed at investigation of the short- and long-term behavior of internally heated pools of reactor materials. The studies include attempts to determine the thermal and kinetic behavior as well as the interaction of the pool with retention materials. For example, fuel steel mixtures would be expected to segregate, significantly affecting heat transfer and interaction potential. The only way in which the selective heating of the $\mathrm{UO}_{2}$ in a steel mixture can be accomplished is through fission heating.

An important consideration is the determination of the patterns of thermal energy flow. The greater the downward heat flux, the more the structure supporting the pool is attacked. Therefore, the first goal of the molten pool program has been to develop a versatile experiment in which heat flux and structural ablation can be studied using real materials under typical temperature and heating conditions.

To achieve the above aims, a high-temperature, out-of-core material compatibility study was conducted to ascertain materials which would contain or insulate under the extremes of temperature and radiation present in the molten pool experiment. Our ultrasonic thermometry system was developed to measure fuel temperatures through melting of the $\mathrm{UO}_{2}$ and to determine the temperature gradients within the hot fuel. The majority of the experiment design was accomplished prior to this quarter.

During this reporting period, the design, drafting, and ordering of the in-core containment vessels were completed. The ultrasonic thermometers, pressure transducers, and seals were qualified in several in-core experiments. Fabrication of the ultrasonic thermonetry electronics modules has begun. The experiment plan was written for submission to the Annular Core Pulsed Reactor Committee. The first fuel-only (no steel), in-core experiment is on schedule for the last of June 1977. 
For every sodium cooled reactor, where a primary system accident would involve a risk to the public health and safety, the release of sodium from the primary system must be considered. This task is designed to provide a large-scale sodium system suitable for studying the interactions of sodium with containment materials and resolving material interaction and compatibility questions related to sodium releases. Current objectives are to produce relatively large-scale sodium/concrete and sodium/liner/concrete interactions in order to provide the data base for determining the controling processes associated with these types of interactions.

The basic test facility is now 80 percent complete. Most components have arrived and no critical problems have been identified. Sixteen prototypic concrete crucibles have completed the 90-day cure and are ready for the experiments. The data gathered from the crucible preparation suggest the concrete specifications for water content should be reviewed.

A draft test plan has been prepared and provided to NRC for comment. Other analytical progress includes making the CACECO code operational at Sandia and determining the definition and execution of the cell liner structural study.

\section{Elevated Temperature Design Criteria}

The central objectives of this task are: (1) to determine the feasibility of detecting elevated temperature damage (creep, low-cycle fatigue, and combined creep-fatigue, by nondestructive means, in class 1 reactor components; (2) to develop improved theories of elevated temperature damage, based upon field theoretic quantities (stress, strain, temperature, time), and provide experimental data by which to evaluate these and other damage theories; (3) to provide an assessment of analytical techniques used to calculate elevated temperature structural instability and to determine the need for experimental verification of these techniques, apart from current efforts by USERDA and foreign laboratories; and (4) to provide analytical treatment of thermal stresses and strains in equipment cell liners due to accidental sodium spills.

To date, subtask 1 has been able to demonstrate a correlation between creep damage in 2-1/4 Cr - 1 Mo specimens that will later be ultrasonically examined. A test matrix for AISI 316 stainless steel is being planned. This entire test program will be completed and assessed prior to a decision point on the surveillance test facility, where fieldapplicable nondestructive testing techniques will be used on prototypic hardware. 
Subtask 2 has evolved to the point that a preliminary design has been completed on a cylindrical, multiaxially loaded test specimen. Equipment for this multiaxial creepfatigue facility has been ordered from the manufacturer. Failure theories proposed by Professors K. J. Miller, S. Y. Zamrik, and others have been examined and a unified theory of strain-range partitioning, employing these ideas, has been formulated.

Subtask 3 is in the early stages of work; a geometry for a piping elbow tested by the Japanese has been obtained and preliminary analys is is underway.

Subtask 4 is approximately 50 percent complete. The study consists of a parametric analys is of early-time thermal shock of a steel liner subjected to a hot sodium spill. The parameters are: the effective heat transfer coefficient of the sodium to steel flux; the effective radius of the spill; and the thickness of the liner compared to distance between liner anchors.

\section{Future Test Technology}

As part of the development of a long-range safety research program for NRC, Sandia is developing the required test technology to provide an increasingly more complete capability to conduct phenomenological experiments both in-pile and out-of-pile. The first such activity is the conduct of a major upgrade to the ACPR. This upgrade will provide approximately a factor of three increase in pulsed power and will provide sufficient fluence to conduct multi-pin experiments as well as higher power, steady-state fuel dynamics and PAHR experiments. The project is jointly funded by ERDA and NRC and is in the final hardware fabrication stage. Progress of this activity is reported in a separate report.

\section{Ful1-Length Capabilities}

The development of a facility at Sandia capable of accommodating ful1-1ength fuel pins and larger bundle sizes than currently possible continues to be under study. Included in this activity are studies of the need for "full-length" tests and conceptual designs of a new reactor system utilizing the existing Sandia Engineering Reactor Facility. During this reporting period, a study was conducted, utilizing MELT-3A, of the effect of a less-thanprototypic fuel pin on a FFTF TOP accident. The results of this study were:

(1) A single, shortened subassembly does not significantly affect the reactor power profile or worth curves.

(2) The preconditioning at peak burnup shows a marked difference due to the fact that the shorter fuel length runs hotter. As a result, the amount of fission gas 
retained is less for the shorter fuel length, and there is a higher tangential stress inside the cladding as a result of enhanced fuel expansion at the higher tenperatures.

(3) The shorter fuel fails at the top of the fuel region at 0.604 second, while the longer fails halfway between the fuel midplane and the top at 0.6115 second.

(4) At and after fuel failure, there is more molten fuel and higher pressure within the short fuel length pins. As a result, more fuel is ejected into the coolant channel. There appears to be no fuel plugging, and the fuel motion induces negative feedback in both cases.

It should be noted that these results apply to the case of a test subassembly whose fuel length is less than that of the driver core. If a driver core had been chosen to be equal in fuel length to that of the test subassembly in both cases, it might have been possible to achieve near-prototypic response through careful selection of the power profile and steady-state power. Investigations in this area will continue.

To achieve a capability to conduct full-length tests, several design concepts are being evaluated. Both water-cooled and gas-cooled systems are under study. Requirements are aimed at achieving the maximum flexibility for testing at a minimum capital cost. Calculations for a $\mathrm{Nb}-20 \mathrm{~W} / \mathrm{O} \mathrm{U}$ and $\mathrm{Be}-\mathrm{UO}_{2}$ system were conducted during this reporting reporting period.

\section{Large-Scale Tests}

A major limitation to safety research is the inability to conduct large-scale (subassembly and above) tests due to a lack of test facilities. Studies are being conducted on the feasibility of utilizing the Nation's underground nuclear test technology at the Nevada Test Site to provide this capability. This highly developed technology may have the potential of providing totally contained, large-scale tests at a fraction of the cost of the development of large-scale test reactor facilities. Tests to address prompt burst energetics, transition phase recriticality, post-accident heat removal, and aerosol source production are being considered. Designs of external source-driven, selfdriven, and hybrid tests are included in the study. Results to date indicate that relative inexpensive facilities could be rapidly developed to provide large-scale test data on a reasonable time scale. 
-

$\bullet$ 


\section{ACCIDENT ENERGETICS}

\subsection{Prompt Burst Excursion (PBE) Experiment}

(T. R. Schmidt, 5451; D. C. Williams, 5425;

M. F. Young, 5425; K. O. Reil, 5422; R. M. Elrick, 5422)

\subsubsection{Introduction}

Prompt Burst Excursion (PBE) experiments are designed to provide information on the energetics of IMFBR cores subjected to hypothetical superprompt critica.I excursions. The Annular Core Pulse Reactor (ACPR) will be used to pulse-heat heavily instrumented fuel pins with and without sodium with initial reactor periods of $1.5 \mathrm{~ms}$ well into fuel vaporization. These experiments are directed to the phases of core disruptive accidents (CDA's), where rapid energy generation occurs with the core losing much of its geometry, and to the energy partition phase, where a fraction of the thermal energy deposited in the fuel is converted to mechanical work. Pressure, temperature, and piston displacement histories will be determined for a variety of fuel types and initial conditions. The experiments examine the integral effects of fuel-coolant-clad interactions, fission gas release, and fuel and fission product vapor pressures for a prompt critical CDA. An initial series of eight experiments is planned for fresh $\mathrm{UO}_{2}$ fuel pins.

A complete description of the experiment hardware was given in the February 1975 quarterly report. ${ }^{1}$

Activities this period were devoted to the continued analysis of the experiments and to preparations for the upcoming experiments.

\subsubsection{PBE Hydrodynamic Analysis}

The analysis of sodium and fuel motion in $\mathrm{PBE}-5 \mathrm{~S}$, reported in the previous quarterly, ${ }^{9}$ has been extended to include motion of the piston in the upper end of the test capsule and heat transfer between fuel and sodium. Principal results are that the calculated bottom/top pressure ratio (1.7) now matches that observed in the PBE-5S experiment (1.52), and that only two pulses are calculated at the top transducer, also matching the experiment. These two results are aue to the moving 
piston; pulses are generated until the piston accelerates to match the local sodium velocity at the piston/sodium interface. The upper sodium slug and piston are then matched in velocity and the pressure at the sodium/piston interface is essentially zero. The problem is now more a flow problem than a shock problem, and, since CSQII includes only hydrodynamic attenuation and viscous dissipation with no frictional loss due to the channel wall, the results become more qualitative in nature. Pressure losses due to wall friction and channel flow changes are on the order of 5-10 psi, however, and do not materially affect the flow problem. Figure 1 shows sodium and piston velocity and pressure at a time after the initial piston acceleration. The velocity drop at " $A$ " is due to a flow area increase at that point in the channel with mass flow remaining constant. Figures 2 and 3 compare the top and bottom pressure traces for the fixed boundary and free piston calculations to experiment. Pressures and time intervals are summarized in Table I. It should be noted that the important relations are the various ratios between pressures and not their absolute magnitudes; for instance, $\mathrm{UO}_{2}$ pressure in the fixed boundary case was 200 bar, whereas that for the free piston was 60 bar. $\mathrm{P}_{\mathrm{UO}_{2}}$ for PBE-5S was inferred from the EXPAND hydrostatic model. The bottom/top pressure ratio is larger for the free piston than the fixed case due to the reduced impedance mismatch at the top boundary. Use of a steel disk (pressure transducer) at the lower boundary rather than the fixed wall would reduce this to about $1.5-1.6$ because of the lower shock impedance, which compares well with the experimental value of 1.52 .

The amplitude decay envelopes of the pulse trains for both CSQII calculations are still much slower than in the experiment. A case was done which included heat transfer in a rather crude fashion (Figs. 4 and 5); the results (Table II) show a much faster decay in pressure than observed in the experiment; the ratio $-\mathrm{P}_{1} / \mathrm{P}_{\mathrm{UO}}-$ is much higher due to the formation of sodium vapor at the break.

The high pressures observed for the heat transfer case are due to the assumption in CSQII of Iocal thermodynamic equilibrium; thus, whenever fuel enters a sodium node, or vice versa, the code adjusts temperatures and pressure to attain local thermodynamic equilibrium over one machine cycle, resulting in an instantaneous jump in temperature and pressure in that node via the sodium equation of state. Pressures generated are generally much greater than would be predicted by a more realistic FCI model and are typical of a Hicks-Menzies approach. Whereas in the free piston case the channel void created by the moving piston filled with $\mathrm{UO}_{2}$ vapor, in the heat transfer case it filled with sodium vapor; in both cases, gross movement of the fuel inside the pin was very slight up to the time the 


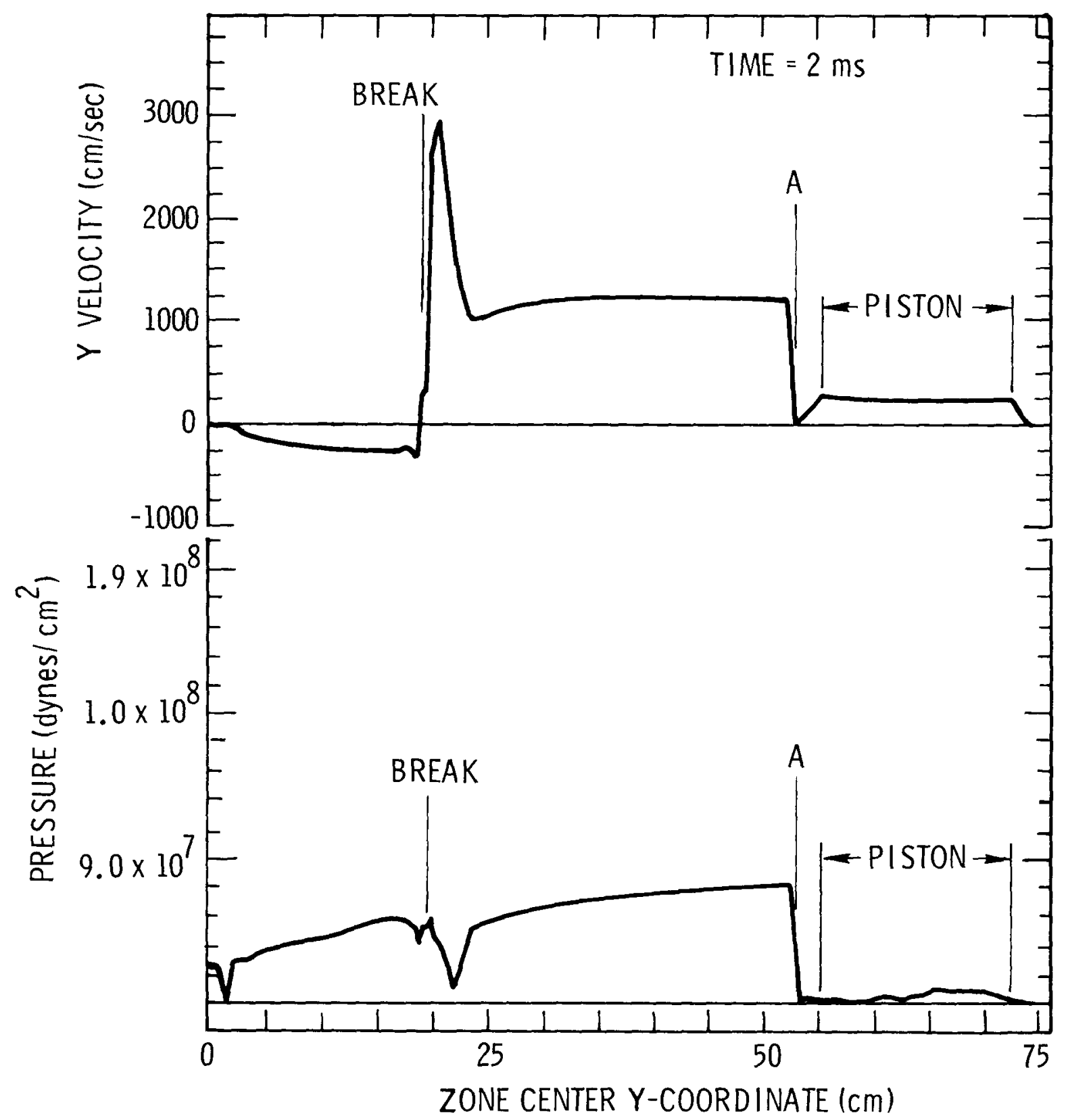

Figure 1. Sodium and Piston Velocity and Pressure 


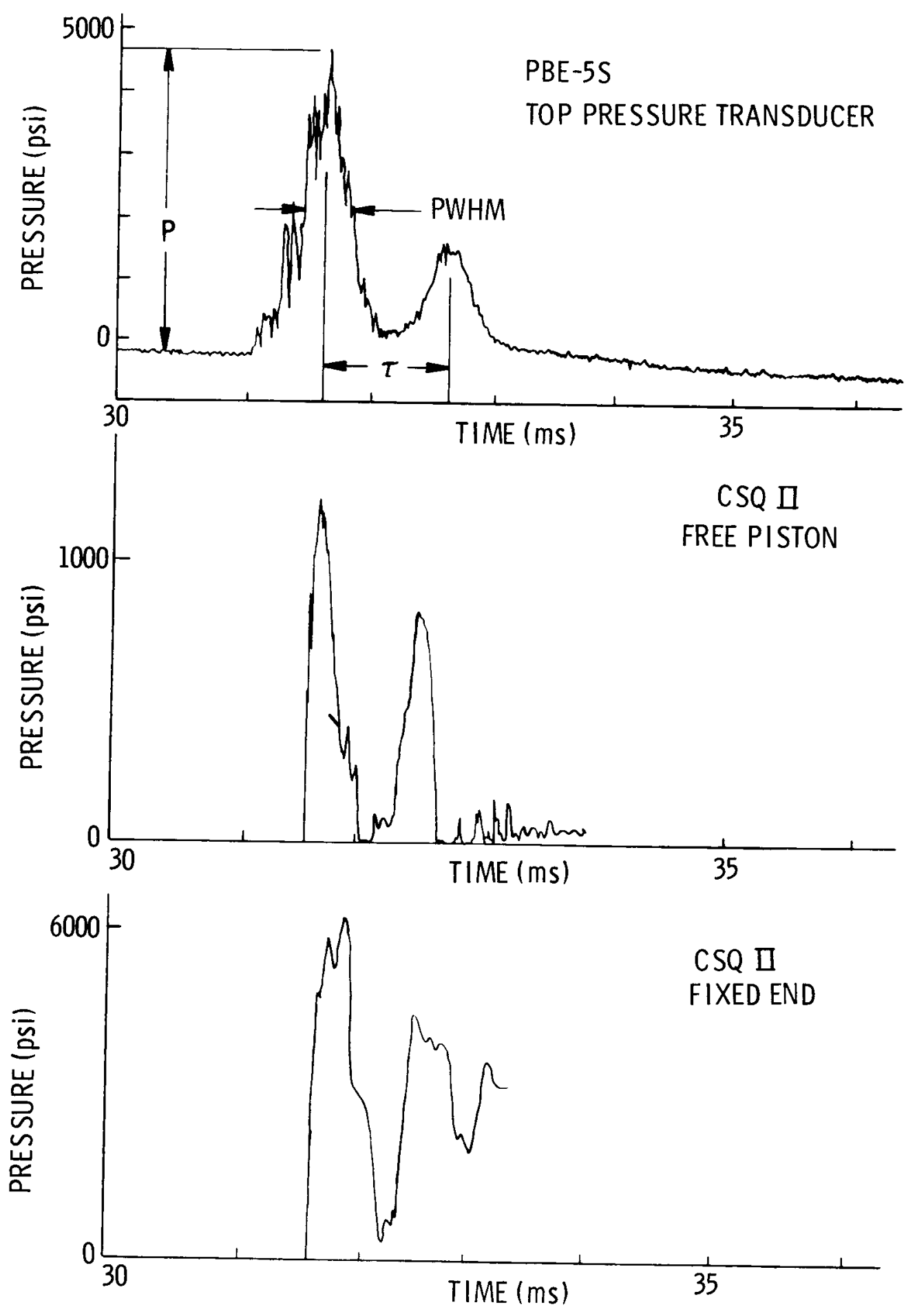

Figure 2. Top Pressure Transducer 

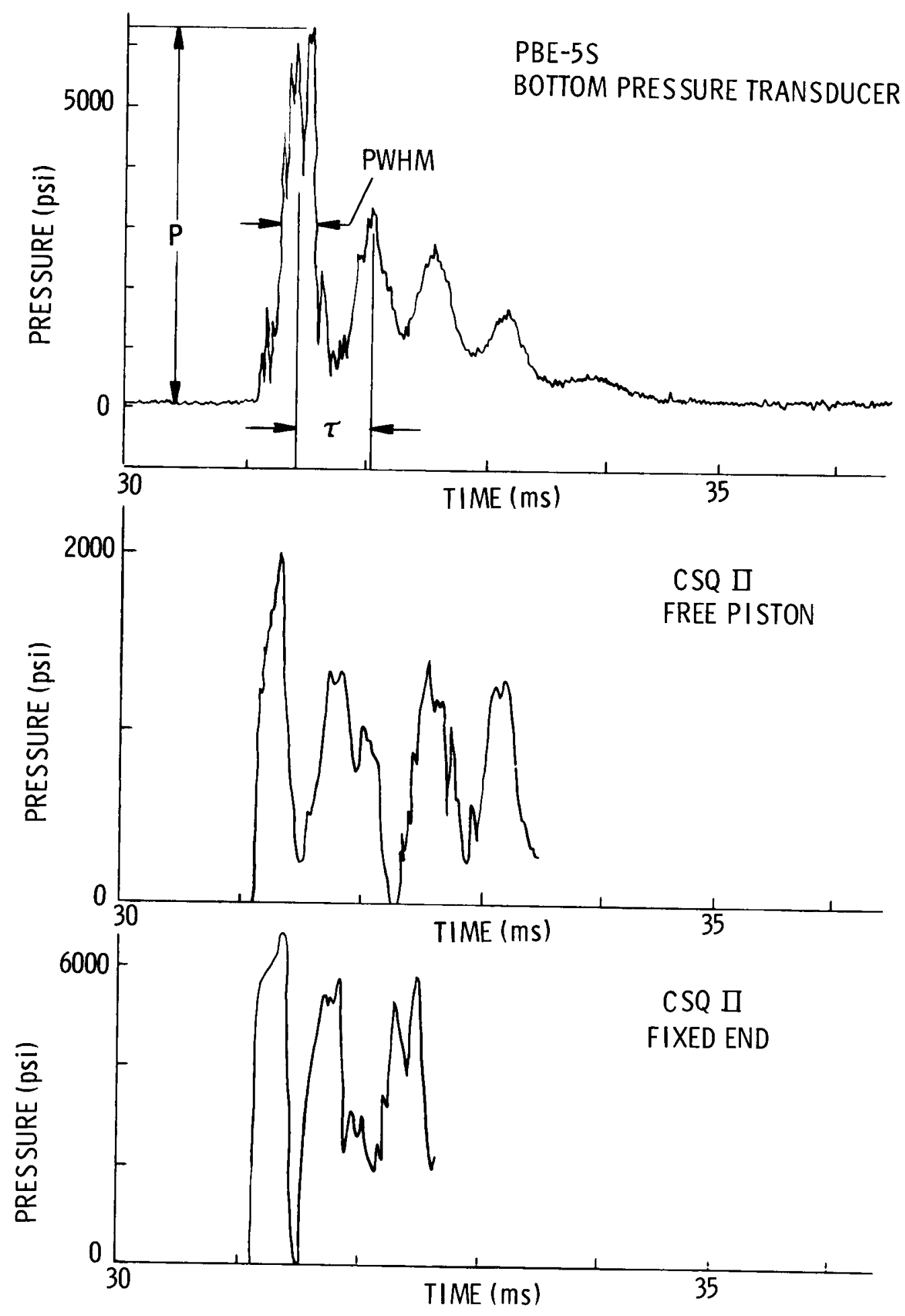

Figure 3. Bottom Pressure Transducer 
TABLE I

Comparison of PBE-5S and Hydrodynamic Calculations

\begin{tabular}{|c|c|c|c|c|c|c|}
\hline \multirow[b]{2}{*}{ First Peak } & \multicolumn{2}{|c|}{$\mathrm{PBE}-5 \mathrm{~S}$} & \multicolumn{2}{|c|}{ CSQII FREE } & \multicolumn{2}{|c|}{ CSQII FIXED } \\
\hline & Top & Bottom & Top & Bottom & Top & Bottom \\
\hline$P_{1}(p s i)$ & 4640.0 & 6220.0 & 1230.0 & 2050.0 & 6380.0 & 6670.0 \\
\hline PWHM (ms) & 0.35 & 0.3 & 0.2 & 0.24 & 0.37 & 0.2 \\
\hline
\end{tabular}

Second Peak

$$
P_{2}(p s i)
$$

PWHM (ms)

1620.0

3260.0

830.0

1380.0

4930.0

5950.0

0.35

0.35

0.24

0.32

0.4

0.24

$\mathrm{P}_{2} / \mathrm{P}_{1}$

0.35

0.52

0.67

0.67

0.77

0.89

$\mathrm{P}_{1} / \mathrm{P}_{\mathrm{UO}}$

$\tau$ (ms)

1.6

2.1

1.3

2.2

2.2

2.3

1.02

0.6

0.8

0.5

0.7

0.4

Bottom/Top

Pressure Ratio:

1.52

1.7

1.1 


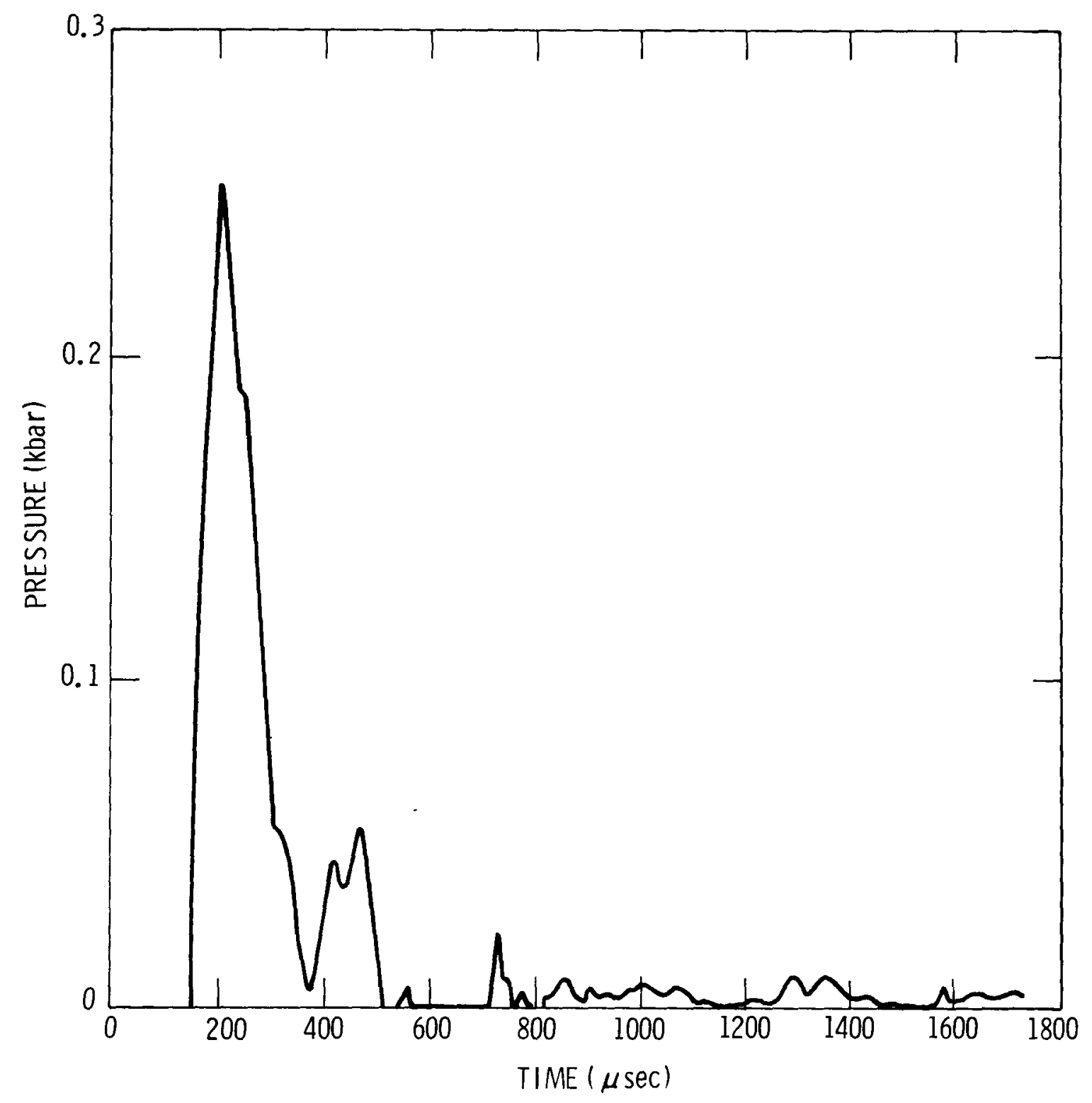

Figure 4. Top Pressure Transaucer for Heat Transfer Case 


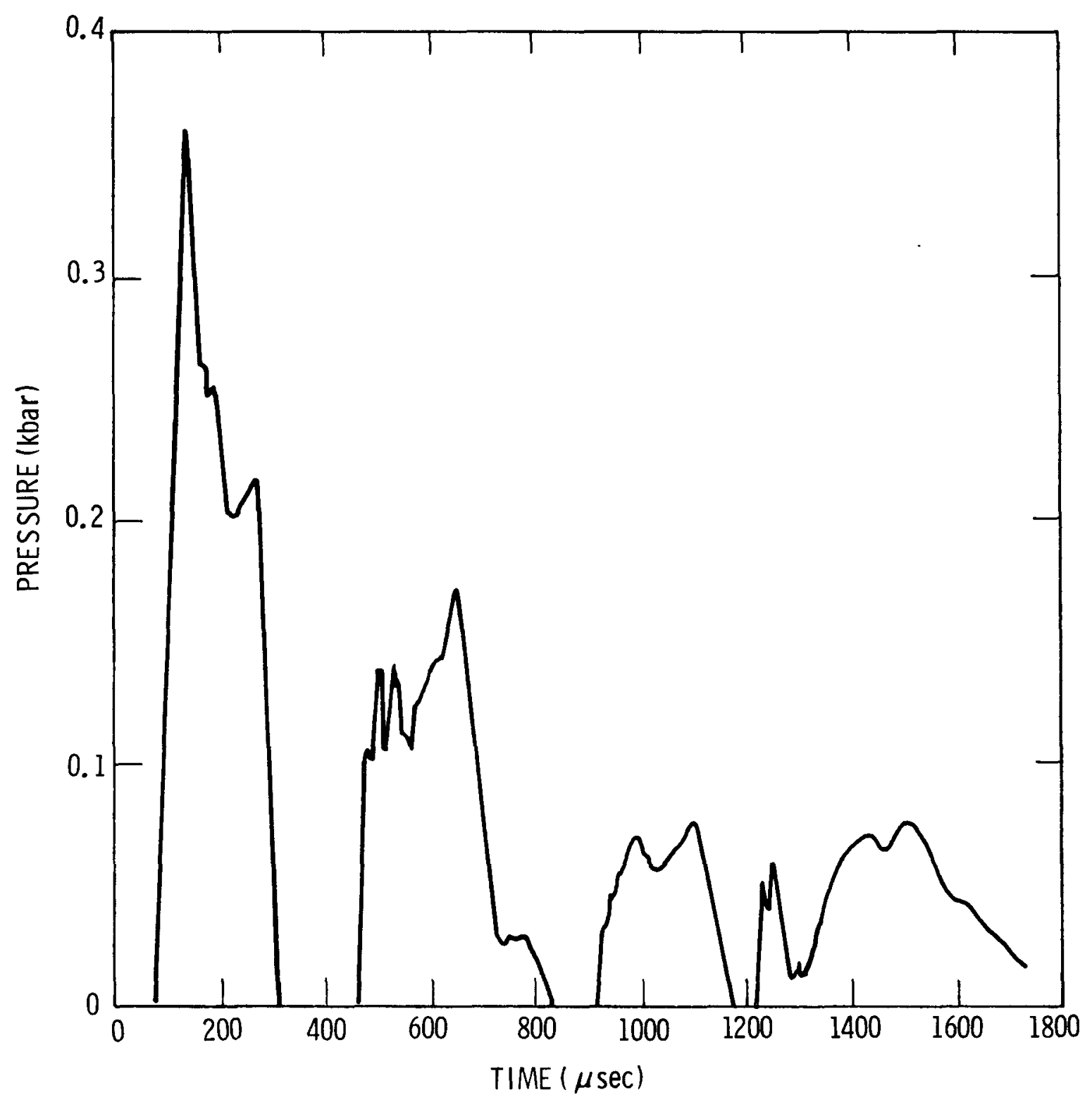

Figure 5. Bottom Pressure Transducer for Heat Transfer Case 
TABLE II

CSQII Heat Transfer Results

CSQII Heat

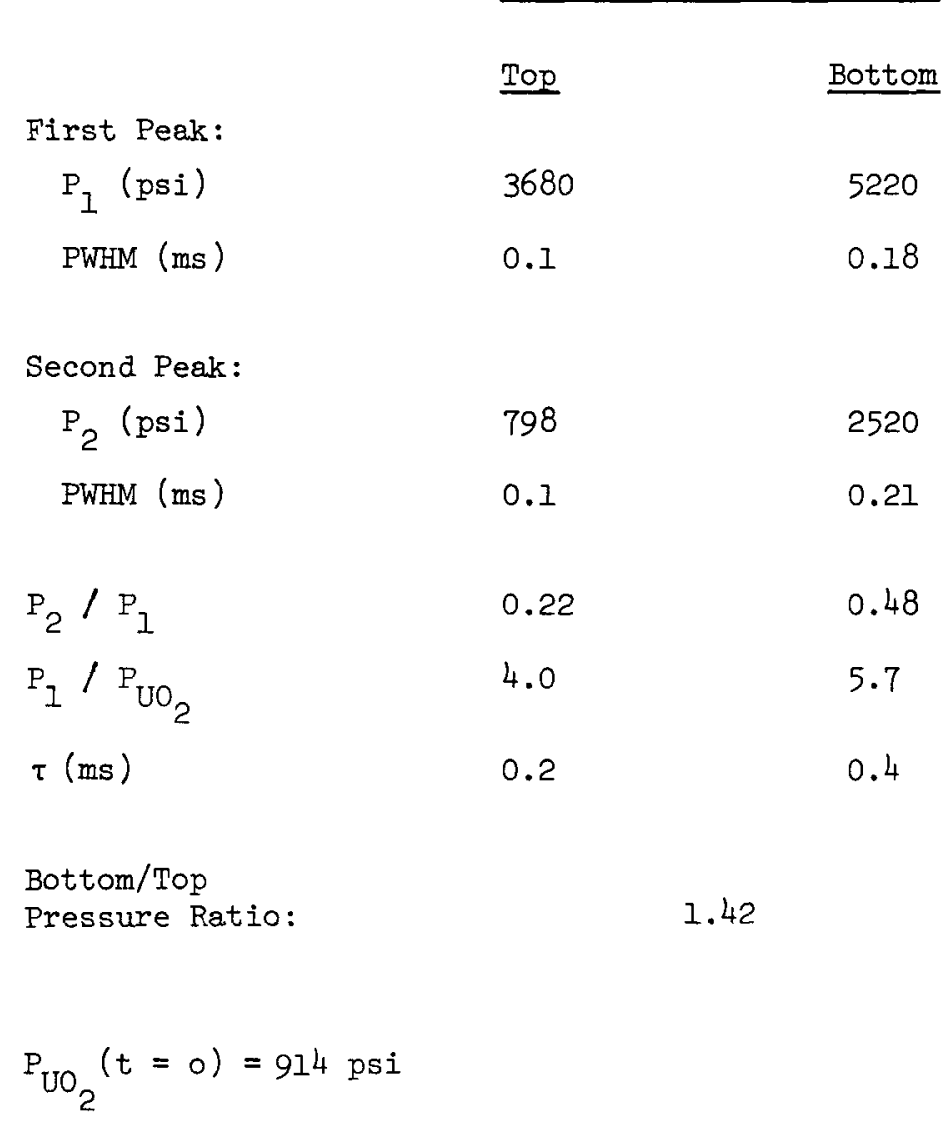


calculations were terminated. It was felt that either the voiding of the upper and lower portions of the cladding observed experimentaliy must occur at later times (10's of milliseconds) or that the crude heat transfer in CSQII resulted in cooling the fuel inside the pin too rapidly. Since running CSQII to longer times would be prohibitively expensive, it was decided to try approaching the problem with a more specialized code such as EPIC. This code has been acquired from ANL and is now being adopted to the Sandia CDC system.

Further analysis will include a CSQII run incorporating energy deposition as well as heat transfer; this should result in a pressure decay rate somewhere between the heat transfer and adiabatic cases. EPIC will be used to follow sodium and fuel motion at longer times than is feasible with CSQII. The EXPAND pin model is being revised with a more accurate clad deformation model and a new vapor pressure and fuel motion calculation. A simple fuel cracking model is also included.

\subsubsection{Experiments}

The PBE-7S experiment was conducted in late December 1976 and involved a $20 \%$ enriched fuel pin in sodium subjected to a single maximum pulse from isothermal initial conditions of $500^{\circ} \mathrm{C}$. This experiment is a repeat of the $\mathrm{PBE}-6 \mathrm{~S}$ experiment where the pressure histories displayed an early event, followed several milliseconds later by a large pressure event generally thought to be associated with gross pin failure. Figures 6 and 7 give the pressure histories from the top and bottom of the PBE-7S experiment capsule. The bottom pressure history is very similar to the PBE-4S and -5S experiments with a peak pressure of $5400 \mathrm{psi}$. The top pressure history, however, shows an initial pressurization nearly coincident with the large signal at the bottom followed two milliseconds later by a large, singlepressure pulse with a peak pressure of 3910 psi. The kinetic energy of the piston was $19.7 \mathrm{~J}$ and the energy conversion ratio is approximately $10^{-4}$. These values are similar to PBE-6S. The radiographs of the two experiments are also similar. Figure 8 is the radiograph from PBE-7S.

\section{1 .4 Related Activities}

The design for the experiment hardware for the PNL-3 fuel pins has been finalized and orders placed. 


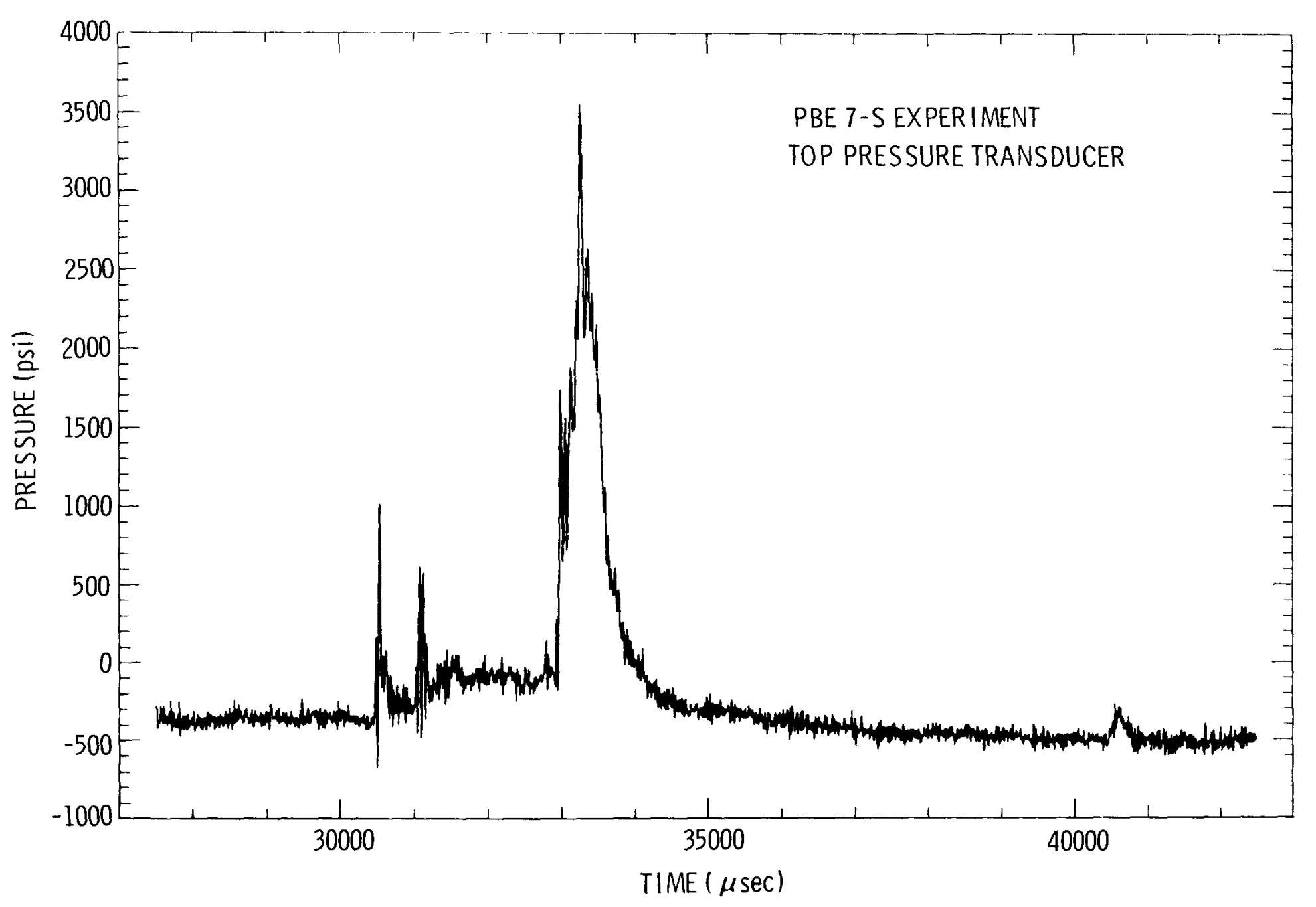

Figure 6. Pressure History at the Top of the PBE-7S Experiment 


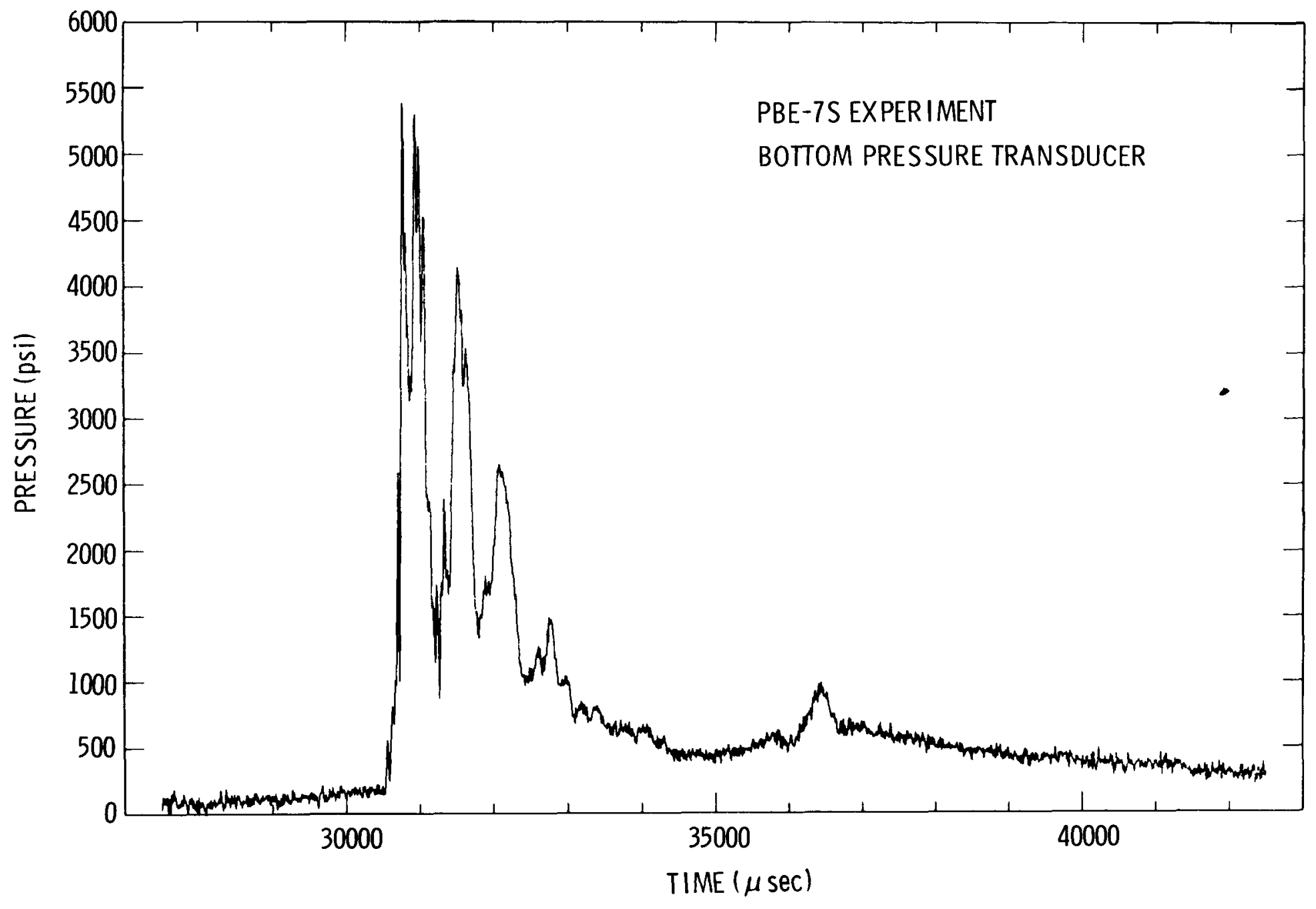

Figure 7. Pressure History at the Bottom of the PBE-7S Experiment 


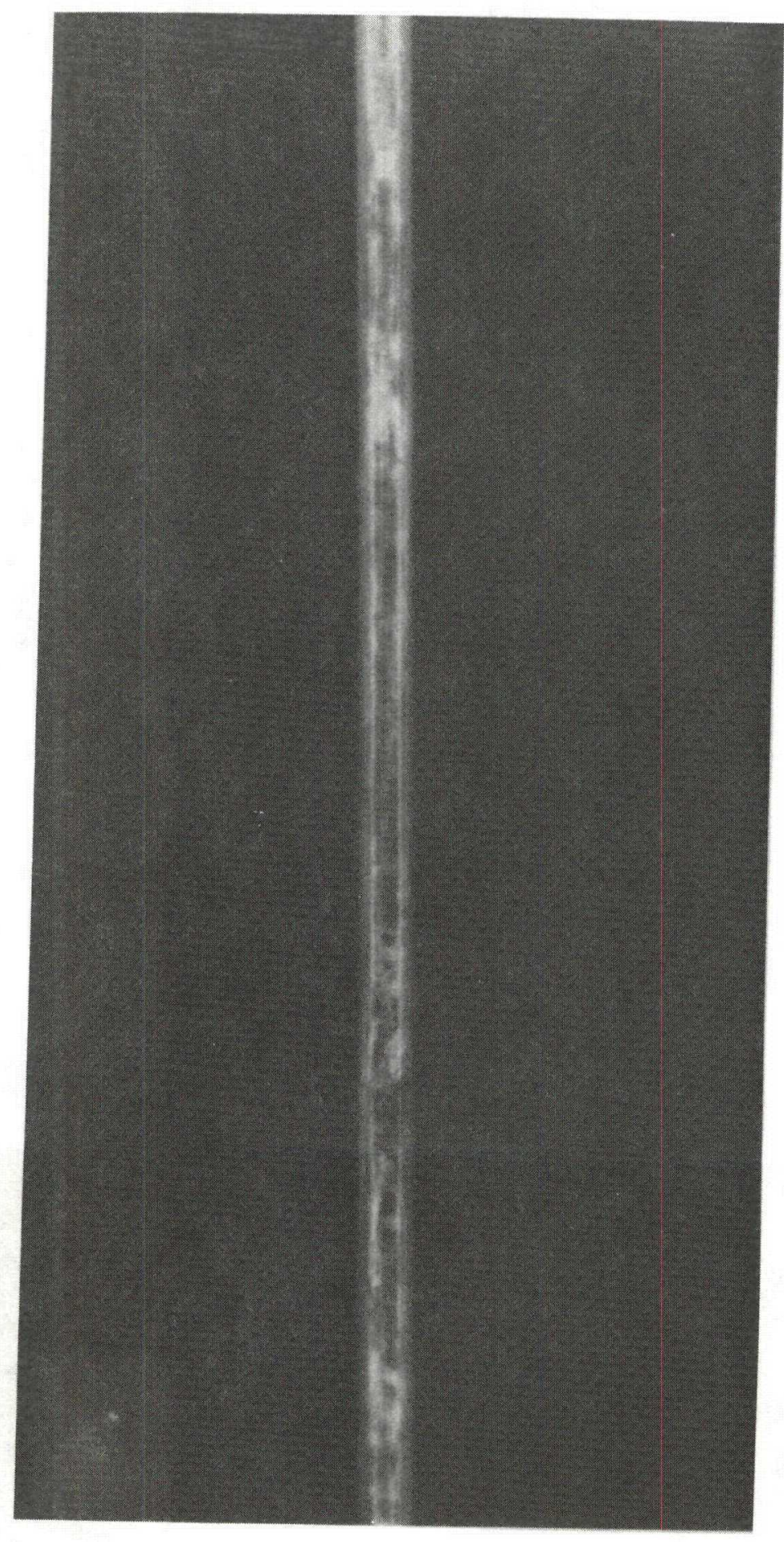

Figure 8. Radiograph of the PBE-7S Experiment 
The fabrication of the new higher reactivity worth transient rods for the $A C P R$ is proceeding. LASL has made test pressings of the $B_{4} C$ material and has achieved densities of 93\% T.D. After the pressings are completed, the material will be sent to General Atomics for fabrication of the control rods.

Figure 9 is a graph of the particle size distribution from two PBE experiments and two experiments, $\mathrm{S} 3$ and E2, conducted in the TREAT reactor. Particle size distributions from other sources are described more fully in a previous quarterly report. 4 The PBE-4S experiment contained sodium while the PBE-3E experiment was dry. Both TREAT experiments contained sodium. In the PBE-3E and $-4 S$ experiments, $20.8 \mathrm{~g}$ and $41.7 \mathrm{~g}$, respectively, of the $64 \mathrm{~g}$ in the fuel column were recovered and included in the graph. The balance of the material was condensed on container walls, remained in unmelted cladding or formed a large plug in the bottom (3E). Including the total mass of the fuel column would move the PBI distributions to the left with the PBE-4S results falling close to the $\mathrm{S} 3$ results.

\subsubsection{Pressure Gage Calibration}

In the PBE experiment, pressure gages are a primary source of information for following the energy exchange between the vaporized fuel and the sodium coolant. The purpose of this study is to estimate the relation between pressure gage response and source pressure, modified by the geometry of the experiment. Modification of the pressure pulse as it travels through the sodium-filled annulus will be studied out-of-pile in the PBE geometry using a well-defined shock wave as the pressure source. The shock wave will be produced by detonating high explosive in water to improve the impedance match into the PBE system. This study is a simplification of events in the PBE experiment since here the pulse propagation is uncomplicated by sodium voiding caused by injection of the fuel vapor. This simplification should allow a well-defined source pressure to be unfolded from the measured pressure and permit some estimate of the motion of the effective mixing front. Within the PBE system, the pressure pulse is measured at three positions along the annulus, including those gage positions in the PBE experiments. At two of the positions, the response of the normally used eddy current gage will be compared with that of a piezoresistive gage. 


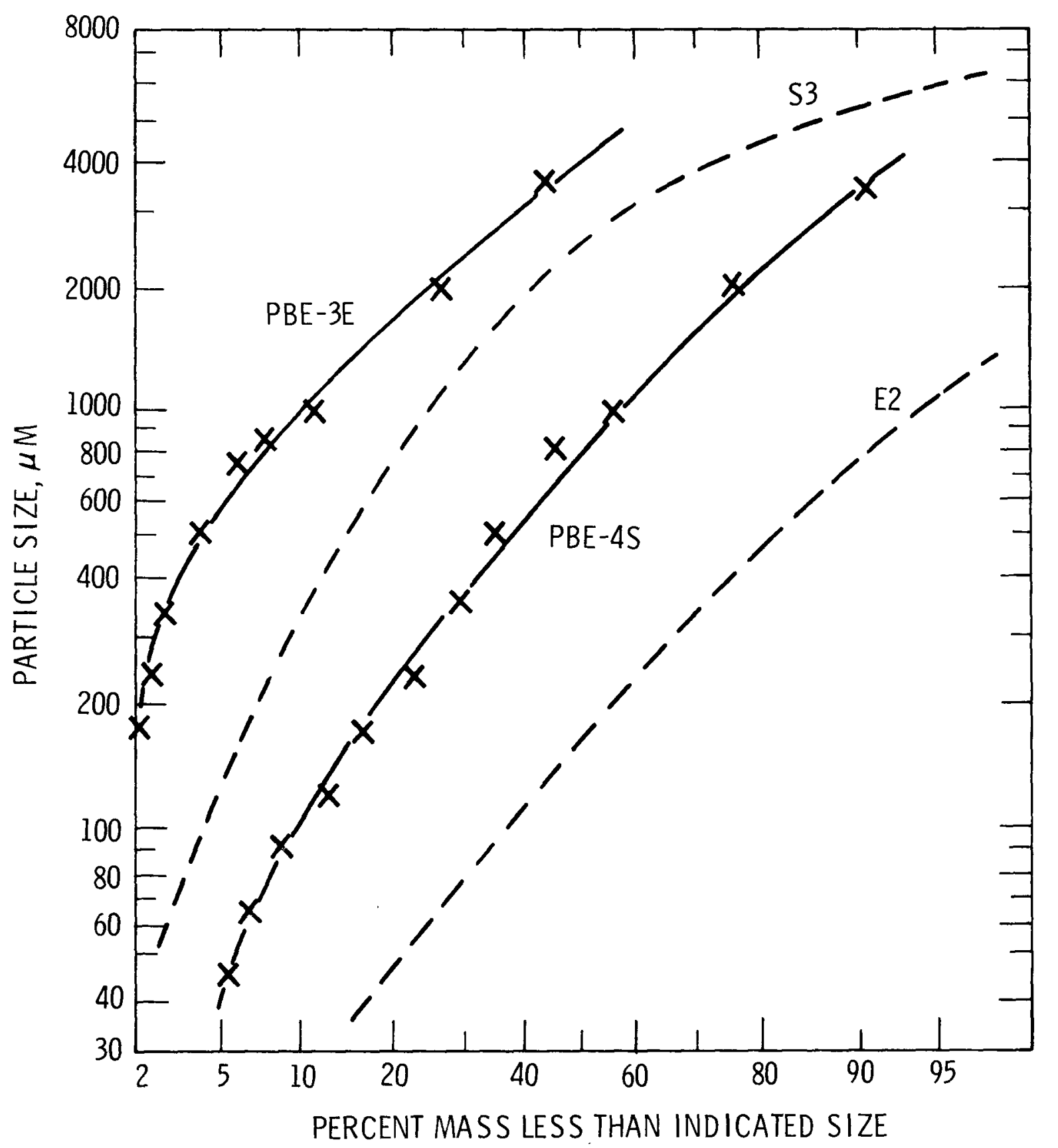

Figure 9. Particle size Distribution from the PBE-3E and $4 S$ Experiments and the $\mathrm{S} 3$ and $\mathrm{E} 2$ Experiments 
During the last quarter, the design, layout drawings, and detailed drawings were completed for the calibration study, and fabrication of the parts was begun. One proof test was shot in a water tank to examine the attenuation, broadening and general shape of the shock pulse. The equivalent of $3 \mathrm{~kg}$ of TNT was detonated at the bottom of a 3-m diameter tank filled with water. Five meters above the detonation, the stagnation pressure was 1700 psi with a pulse width of 300 us. Since the shock pulse should be at least as long as a PBE pulse ( I ms) and with a similar amplitude ( 5000 psi), several proof tests are scheduled for next quarter in an attempt to generate stronger and longer duration pulses in smaller diameter pipes. Also during the next quarter, fabrication of system parts will be completed. The experiment will be assembled and checked, and a series of shock propagation measurements will be made in the system using glycerine, with a sound speed similar to sodium, as a test fluid.

\section{1 .6 Status}

Activities for the next period will continue with analysis of the experiments and preparations for the coming experiments. A series of dosimetry experiments will be completed.

\subsection{Equation of state}

(D. A. Benson, 5167; E. G. Bergeron, 5425;

K. O Rei1, 5422; B. M. Butcher, 5167; E. I. Wilmot, 3312)

\subsubsection{Electron Beam Vaporization Studies}

Comparison of the dynamic vaporization data from electron beam experiments has been made in the past to extrapolations based upon conventional vapor pressure and heat capacity data. The predictions based upon conventional data produce vapor pressure estimates which are one order of magnitude below those of the dynamic measurements for a given energy state. Additional data to illuminate this comparison have now been obtained. 
The dynamic vaporization data from the electron beam source have recently been confirmed by new results from vaporization experiments in the ACPR reactor. In addition to those vaporization experiments, a second confirmation can be found in reactor experiments carried out on dynamically heated single fuel pins in sodiumfilled capsules (for example, the TREAT S-11 on PBE-5S tests). Peak pressures observed in those tests, when expressed as a function of fuel interval energy, also compare well with the previous data. Thus dynamic vaporization results from three diverse experiments show similar vaporization properties which differ from predictions based upon conventional data.

A significant difference between the conventional and dynamic experimental regimes appears to be one of heating time. This suggests the existence of important rate effects in sudden heating of $\mathrm{UO}_{2}$. The calculation of transient reactor phenomena should be based upon information valid at high heating rates rather than the conventional data. To examine the significance of such an error, initial calculations of the disassembly and expansion process using the two types of equations of state have been carried out. These computations used the one-dimensional hydrodynamic $S_{n}$ code, PAD, developed at Los Alamos. The reactor nodel chosen was that of a $1000 \mathrm{MWt}$ two-zone core and blanket geometry. Work release from an 8-fold expansion following disassembly calculated in this model differs by a factor of two to ten, depending on the choice of equation of state.

\section{2 .2 Heat Capacity $\mathrm{UO}_{2}$}

The initial stages of a calculation of the anharmonic contribution to the heat capacity of $\mathrm{UO}_{2}$ have been completed. It has been determined that a BornMayer type of interatomic potential will be used, the relevant interaction parameters being the same as those used in the surface energy and heat of formation calculation of Benson, Freeman and Dempsey. 10 The heat capacity calculation will be based on the anharmonic Einstein model used for argon by Henkel ${ }^{11}$ and others and will be checked against thermal expansion data and the elastic constant data of Dolling, et al. ${ }^{12}$ Significant progress on the actual calculation has been made with completion tentatively planned for next quarter.

The anharmonic heat capacity calculation is a part of a general EOS investigation for $\mathrm{UO}_{2}$. As an additional part of this investigation, efforts have been made 
to understand the circumstances under which forced congruent evaporation of $\mathrm{UO}_{2}$ takes place and what effect such evaporation has on the vapor pressure of the evaporating species. It is anticipated that present analyses of PBE experiments at Sandia and laser pulse heating experiments elsewhere may have to be reevaluated to include such effects.

\subsubsection{ACPR Effective Equation of State}

During this quarter the analysis of the data from all previous ACPR fuel vaporization experiments was completed. For each series of experiments, a pressure history and four different energy histories are derived. The average energy input history, $E_{A V G}$, is derived by normalizing the integral of reactor power to the total fission energy deposition in the sample as determined by fission product inventory techniques. An upper bound on the energy content (designated $E_{P E A K}$ ) is obtained by scaling $E_{A V G}$ by the peak to average fission density ratio determined from two-dimensional neutron transport calculations. (For the 30\% enriched samples irradiated with a $27.5 \mathrm{~mm}$ thick polyethylene moderator, the peak-to-average ratio is 1.19). Energy Iosses, $E_{\text {LOSS }}$, from the sample were derived by integration of heat fluxes over the inside surface area of the graphite crucible (see reference 13). The heat fluxes were estimated from transient thermocouple measurements using the inverse heat conduction technique described by Beck. ${ }^{14}$ Finally a lower bound on the maximum energy content of the sample is obtained as the difference between $E_{A V G}$ and $E_{\text {LOSS }}$. This corrected average energy content is designated $E_{A C}$. Typical pressure and energy histories for a complete series of EEOS measurements are shown in Figure 10. In this experiment a $30 \%$ enriched $\mathrm{UO}_{2.08}$ sample was irradiated with a $27.5-\mathrm{mm}$ thick polyethylene moderator during a maximum ACPR pulse. of the resulting $2720 \mathrm{~J} / \mathrm{g}$ energy deposition, approximately 83 percent was deposited during the prompt pulse with the balance associated with the pulse tail.

Bounds on the vapor pressure, of the fuel material expressed as pressure versus energy content, are obtained by the point-by-point comparison of the pressure history with $E_{P E A K}$ and $E_{A C}$. Because of uncertainities in the data after the pressure peak, only data obtained during the rise of the pressure pulse are used. The results of all the EEOS series are in agreement within experimental uncertainties for the range of energy content common to all series. The final results are shown in Figure 11 as upper and lower energy bounds on the vapor pressure of Un 2.08 

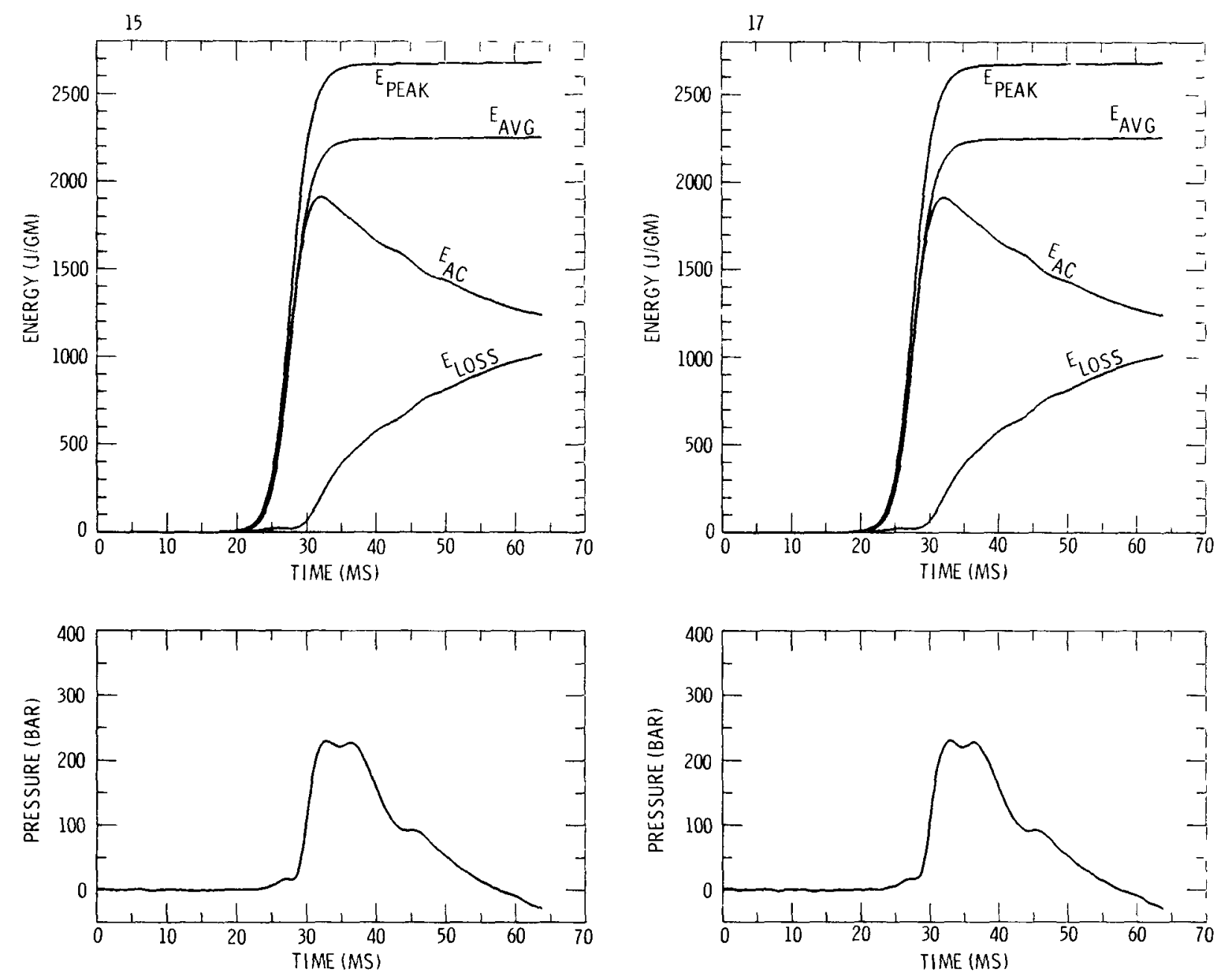

7948-7930 CHAN 3

7930 CHAN 12

7948-7930 CHAN 3

7948 CHAN 12

Figure 10. Pressure and Energy Histories from EEOS-UO2-4 


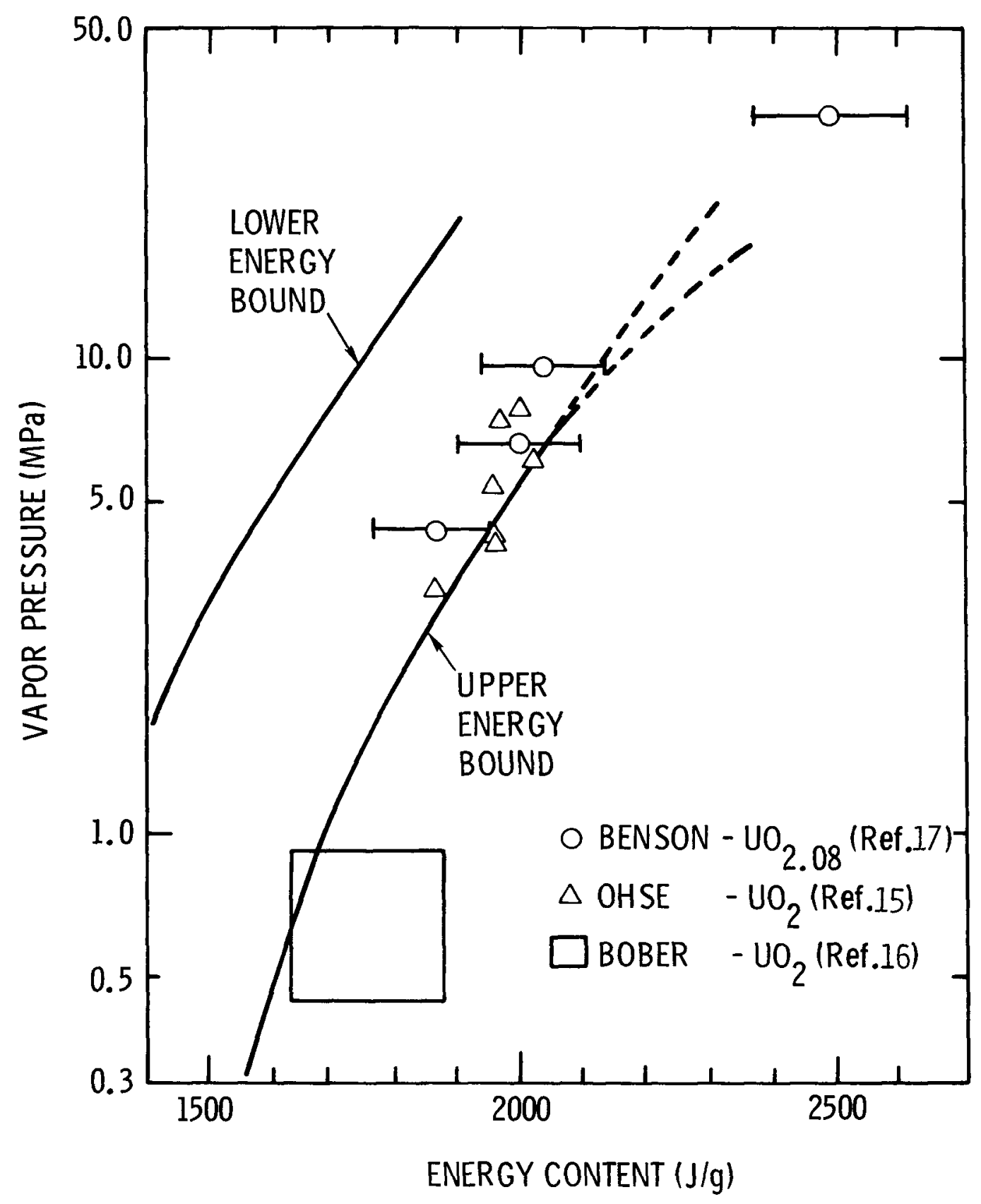

Figure 1l. Comparison of EEOS Results with Other Experimental Data 
Also shown in the figure are results of laser heated experiments $(15,16)$ and electron beam heated experiments (17). Good agreement, particularly between these EEOS results and the electron beam results, is noted.

The separation between the upper and lower energy bounds is a result of the nonuniform energy deposition in the sample; and, hence, is always at least as large as the peak-to-average fission density ratio. Techniques need to be developed to obtain more uniform energy deposition in the sample, and, hence, reduce the separation in the bounds.

\subsubsection{New Experimental Directions}

To further clarify the rate dependent process in $\mathrm{UO}_{2}$, additional experiments have been proposed. The first of these uses the SPR III puised reactor to probe the high temperature states. By varying the sample confinement time, an equation of state in the form of $P(E, V, t)$ can be determined. Such measurements will show details of the time dependent state surface over the scale of interest for reactor transients.

Initial neutron transport calculations using TWOTRAN have been completed which demonstrate that useful dose levels (approaching $1000 \mathrm{cal} / \mathrm{g}$ ) can be achieved in a. SPR experiment for fully enriched $\mathrm{UO}_{2}$ with a l-inch thick polyethylene moderator sleeve. Thick polyethylene moderator sleeves appropriate to this experiment are currently being tested in SPR with dosimetry measurements prior to completing the design of the vaporization experimental package.

As a means of exploring an even wider range of time dependent effects, a second method is being evaluated. For temperatures below the boiling point, direct measurements of the heat capacity at high heating rates may be possible. A choice between reactor and electrically heated samples for this purpose is being studied.

The measurements described above should allow a complete description of the time dependent EOS properties needed for reactor disassembly analysis. 


\subsection{Transition Phase Studies}

(R. W. Ostensen, 5425; R. L. Coats, 5422)

\subsubsection{Introduction}

Transition phase studies are designed to provide information on the behavior of an LMFBR core subjected to a mild core meltdown. In this phase of a loss-offlow accident, at least part of the core has lost its initial geometry, but prompt critical excursions have not occurred or have been too mil.d to permanently disassemble the core. The present effort is devoted to an identification of the important problems, planning of an experimental and analytical program to address those problems, and obtaining relevant computer codes to supplement the experimental and analytical program.

\subsubsection{Identification of Key Problem Areas}

Two key problem areas in the transition phase have been identified. In order of importance these are:

a. fuel blockage formation;

b. boiling pool phenomenology.

Highest priority is being given to the question of the formation of fuel blockages. Such blockages coula occur as molten fuel anà steel are driven from the core into the axial blankets through the voided sodium subchannels between fuel pins. The presence of such blockages would prevent permanent fuel removal from the core. This would lead to a highly complex accident scenario with the possibility of significant prompt critical excursions. If such blockages do not occur and disrupted fuel can be easily and rapidly removed from the core, through the above- or below-core structure, then subsequent recriticalities become highly unlikely and the accident scenario becomes far simpler. Thus the presence or absence of fuel blockages is a key branch point in analysis of a loss-of-flow accident. 
The second problem area, boiling pocl phenomenology, is relevant only if blockages prevent permanent fuel removal. In this area, uncertainties exist with respect to the basic physics of a boiling pool of fuel and steel. Convective heat losses, flow regimes, degree of boil-up as a function of power level, existence or stability of insulating fuel crusts along steel walls, axial void distribution, uniformity of mixing of pool components and dynamic response of such a pool to changes in power level are all in need of investigation.

\subsubsection{Fuel Blockage Formation}

There is controversy in the Iiterature on the mechanism by which hot streaming fuel freezes as it flows through relatively cool steel channels. Analytical models have been based on either a bulk freezing mechanism or a stable crust growth. Although varying agreement has been obtained with experiments, the bulk freezing model apparently predicts experimental results better than the stable crust model. In order to understand the mechanism of freezing, a series of visual streaming and freezing phenomenolog,ical experiments will be conducted using frozen water or other materials (as the steel channel simulant) and cyclohexane or other materials (as the fuel simulant) at varying inlet temperatures. Not only will freezing of the fuel simulant occur but melting of the simulated channel wall can occur. The manner and extent of freezing are dependent on parameters such as the Reynolds number, temperature difference, relative thermal diffusivities, Prandtl Number, critical allowable stress or strain (rates), and possibly other material properties. Therefore, the initial question to be addressed is: What is the freezing mechanism under given flow conditions and how invariant is this mechanism as the flow and simulant materials are changed? Equally important is the determination of the axial penetration as a function of the above parameters. These experiments can simulate fuel crust formation as well as channel wall ablation - effects which have been neglected in some other experimental programs.

It is essential to develop a computational ability to accurately predict a priori the extent of freezing and the axial penetration of the molten fuel as it flows through cool steel channels. It is equally important to develop capability to assess the time scale of melt, movement, and refreezing of frozen plugs due to internal energy generation, large pressure gradients, external thermal attacks, and other likely reasonable effects. As an initial effort toward this end, existing 
computer codes which have been designed or which may be adapted to treat some of the above-mentioned problems will be obtained, studied and modified, as necessary. Programs have been written at Argonne National Laboratory and Brookhaven National Laboratory. In addition, there may be capability in the Los Alamos Scientific Laboratory SIMMER Code and in some in-house Sandia Laboratories codes.

The focal point of the effort on fuel blockage formation is a series of projected large-scale tests, using $\mathrm{UO}_{2}-\mathrm{ZrO}_{2}$ thermite for some tests and pure $\mathrm{UO}_{2}$ for others. The previously described efforts are aimed at providing the ability to accurately predict the results of these large-scale tests. In these tests, large quantitites $(6$ to $30 \mathrm{~kg}$ ) of fuel simulant will be injected under pressure into steel tube bundles of various sizes containing depleted $\mathrm{UO}_{2}$ pellets in a simulated blanket region. The injected fuel can contain various fractions of molten steel and entrained gas. These tests are in the conceptual design stage.

\subsubsection{Boiling Pool Phenomenology}

In the area of boiling pool phenomenology, emphasis has been placed on assessing previous work. Attention has focused on the question of flow regime. One contention is that, for all power levels, the boiling pool will always be dispersed in a subcritical state due to the existence of a dispersed aroplet flow regime. It is felt that more compacted boiling regimes cannot be ruled out. This is because natural convective effects and the related fluid motion are fundamental in defining the boiling flow regime and degree of dispersal. Neglect of these phenomena must result in a nonconservative lower bound for the power levels needed to produce a dispersed droplet flow regime.

Basic experiments and analysis will be performed so that the phenomena which govern the boiling pool flow regime may be understood. A 1.0-kW capacity microwave oven is available and will be used in boiling pool experiments. Attempts will be made to measure the vertical distribution of void fraction in these experiments.

Large-scale experiments are being considered to address the question of crust stability in large boiling pools. These tests are in a conceptual design stage. 
Long-range planning has begun on in-pile experiments using simulant materials. The goal of such tests would be to use simulants with appropriate properties. These tests would involve a volumetrically heated ceramic dispersed by the boiling of an immiscible metal of lighter density than the ceramic. The tests would include ceramic crust formation on the walls as well as melting and ablation of those walls. These tests would be conducted in ACPR with pool behavior monitored by the coded aperture imaging system. Suitable ceramic-metal pairs with the correct properties (e.g., relative melting and boiling points) must be found for such tests to be of value.

\subsection{Aerosol Source Normalization}

(R. M. Elrick, 5422; B. D. Zak, 5443)

\subsubsection{Introduction}

For those reactor accident scenarios in which the fuel vaporizes and ruptures the cladding, it is necessary that the state of the fuel prior and subsequent to rupture be characterized by experiment. The first phase of this study conducted in FY 77 will measure some of the physical properties of $\mathrm{UO}_{2}$ fuel pins heated to vaporization by neutrons from a pulsed reactor. Succeeding work will study the interaction of neutron-vaporized fuels with the containing structure and with sodium.

Debris properties of the $\mathrm{UO}_{2}$ fuel, (such as particle size, velocity, and density distributions as a function of time) will be measured for both bare and clad fuel pins. This characterization will: (a) be used as a particle source term for aerosol codes; (b) be sufficiently accurate and complete for comparing it with hydrodynamic code predictions; and (c) be compared with those particles sampled by similar methods in relevant out-of-pile experiments at Oak Ridge National Laboratory in which the fuel material is resistively heated. This comparison of in-pile to out-of-pile techniques will begin to establish limits of interest on the particle density and size spectra of the vaporized fuel.

A complete description of the experimental design was presented in the previous quarterly report. 9 


\subsubsection{Progress}

During the last quarter, the detailed drawings for the in-pile aerosol. experiment were completed and fabrication of parts has begun.

Within the experimental canister a bare fuel pin will be resistively heated to $\sim 500^{\circ} \mathrm{C}$, vaporized in the pulsed reactor, and the fuel debris collected in a time-resolving particle sampler. Preparation of the sampling wheels is continuing.

The passive, in-reactor vacuum system is designed to hold a pressure of $\sim 10^{-3}$ Torr in the canister which allows the smallest debris particles to travel to the sampler for collection.

High-speed framing photography will be used to determine a time scale for sampling, measure the high fuel pin temperatures, examine the characteristics of the expanding debris front and synchronize the sampling, neutronic and visual event.s. Tools for aligning the optical train in the reactor have also been designed and are being fabricated. Commercial parts for the aerosol experiment are being ordered.

During the next quarter, parts fabrication as well as the sampline wheel preparation will be completed and all systems will be assembled and bench checked.

\subsection{Fuel Coolant Interactions}

Work on this portion of the IMFBR Safety Research program is not scheduled to begin until FY 78 . No activity is reported this quarter.

\subsection{Large-Scale Test Capabilities}

(S. A. Dupree, 523I; D. H. Nguyen, 5425; J. P. Odom, 5423; J.S. Philbin, 5452; J. E. Powell, 5423; I. D. Posey, 5452)

\subsubsection{Full-Length Capabilities}

A facility capable of performing phenomenological experiments with full-length fuel pins under prompt burst and other abnormal conditions continues to be studied. 
It still appears that by utilizing the Sandia Engineering Reactor Facility (SERF), there is a possibility that the facility could be built at a substantial savings and in a relatively short period of time. This facility is to be an integral part of the NRC RSR Experimental Program being carried out at SLA.

The scope of, and requirements for, this experimental program are presently being defined. Other experimental capabilities which appear to be a necessary part of the program are ACPR Upgrade and the use of NTS for large-scale tests. As a result of these considerations, the major test requirements placed upon the above facility are in the small-bundle to partial-subassembly range with prototypic to fully enriched test fuel. This is not to exclude full subassembly tests in such a facility, if it were possible to accommodate them in a facility costing between 20 and 30 million dollars.

The major activity during this quarter has been in the areas of quantifying experimental requirements, investigating prototypicality for specific test conditions, and performing a preliminary conceptual design for the new reactor facility capable of full-length fuel pin tests.

1.6.1.1 Experimental Requirements - Experimental requirements are continuing to receive attention at SIA insofar as the RSR experimental program is concerned. Additional staff has been added and a division formed to address this area. SLA and IASL have been working together to formulate the NRC position concerning fuel motion detection system performance requirements. In order to perform this task, a definition of the types of experiments to be performed is required. Activity in these areas will receive continued emphasis during the next few quarters.

\subsubsection{Test Condition Prototypicality - Prototypicality considerations} continue to be addressed. In particular, the effects of a less-than-prototypic fuel pin length on the course of a TOP situation have been examined for the FFTF environment. In this analysis the pin length for a single FFTF subassembly has been reduced with no significant effect upon the FFTF power profile or reactivity worth. 
Calculations were performed using the MELT-3A computer code. The length of the end reflectors was increased to preserve the total pin length. It must be noted that MELT-3A uses a damage parameter correlation 18 to determine the time and location of fuel failure, thereby eliminating the need to input these parameters. However, this correlation is based on experimental results with ramp rates up to only $\$ 3.00 /$ second. This, then, is the limitation imposed upon the present analysis. other relevant operating conditions are:

\author{
Steady State Power $=384 \mathrm{MW}$ \\ Peak Burnup $=18100 \mathrm{MWD} / \mathrm{MT}$ \\ Peak FIux $(\mathrm{E}>. \mathrm{I} \mathrm{MeV})=4.3 \times 10^{15} \mathrm{n} / \mathrm{cm}^{2}-$ second
}

A TOP analysis, at $\$ 3.00 /$ second, was carried out for the central subassembly for the case of both full fuel length $(91.44 \mathrm{~cm})$ and a shorter case $(60.96 \mathrm{~cm})$. The essential results of analysis are summarized as follows:

a. A single shortened subassembly does not significantly affect the reactor power profile or worth curves.

b. The preconditioning at peak burnup shows a marked difference due to the fact that the shorter fuel length runs hotter. As a result, the amount of fission gas retained is less for the shorter fuel length and there is a higher tangential stress inside the cladding as a result of enhanced fuel expansion at the higher temperatures.

c. The shorter fuel fails at the top of the fuel region at 0.604 second, while the longer fails halfway between the fuel midplane and the top at 0.6115 second.

d. At and after fuel failure, there is more molten fuel and higher pressure within the short fuel length pins. As a result, more 
fuel is ejected into the coolant channel. The reactivity time histories for fuel motion and sodium void are shown in Figure 12. There appears to be no fuel plugging and the fuel motion induces negative feedback in both cases.

It should be noted that these results apply to the case of a test subassembly whose fuel length is less than that of the driver core. If a driver core had been chosen to be equal in fuel length to that of the test subassembly in both cases, it may have been possible to achieve near-prototypic response through careful selection of the power profile and steady-state power. Investigations in this area will continue.

1.6.1.3 New Facility Conceptual Design - Several test-facility design concepts are being evaluated for the High Fluence Fast Pulsed Reactor (HFFPR). A metallic fuel, $\mathrm{Nb}-20 \mathrm{w} / 0 \mathrm{U}$, has been studied extensively for use as a gas-cooled fuel. Although this fuel has some very attractive features (high melt temperature, negative temperature feedback from niobium resonances, and hard spectrum), high enrichments are required to maintain a reasonable core size (< 1.5 meters in height and diameter) and this, in turn, reduces the FOM in fully enriched test fuel to about 5 or 6 . Softening the spectrum with moderators or using this fuel as a convertor are still under consideration.

BeO-UO 2 gas-cooled systems are also being evaluated. Molecular ratios in the region of $15 / 1$ and $20 / 1$ using $50-70 \%$ enriched uranium look promising. These systems have a higher volumetric heat capacity and smaller critical dimensions, but a softer spectrum, than the metal fuels. Nickel-aluminum and iron-aluminum alloy fuels are also being evaluated as possible convertors, should such be required.

Criteria have been established for relating fissile loading and FOM performance in gas-cooled system. The criteria are based on the assumption that energy deposition per fissile atom is the same in the driver and the test fuel. The result is an expression for the fissile atom fraction of the driver, $C_{D}$, as a function of the desired temperature ratio of the target and driver. 19 This leads to criteria for determining fissile atom fractions in the driver fuel required to produce design depositions in prototypic or enriched test fuel. If the driver fuel temperature 


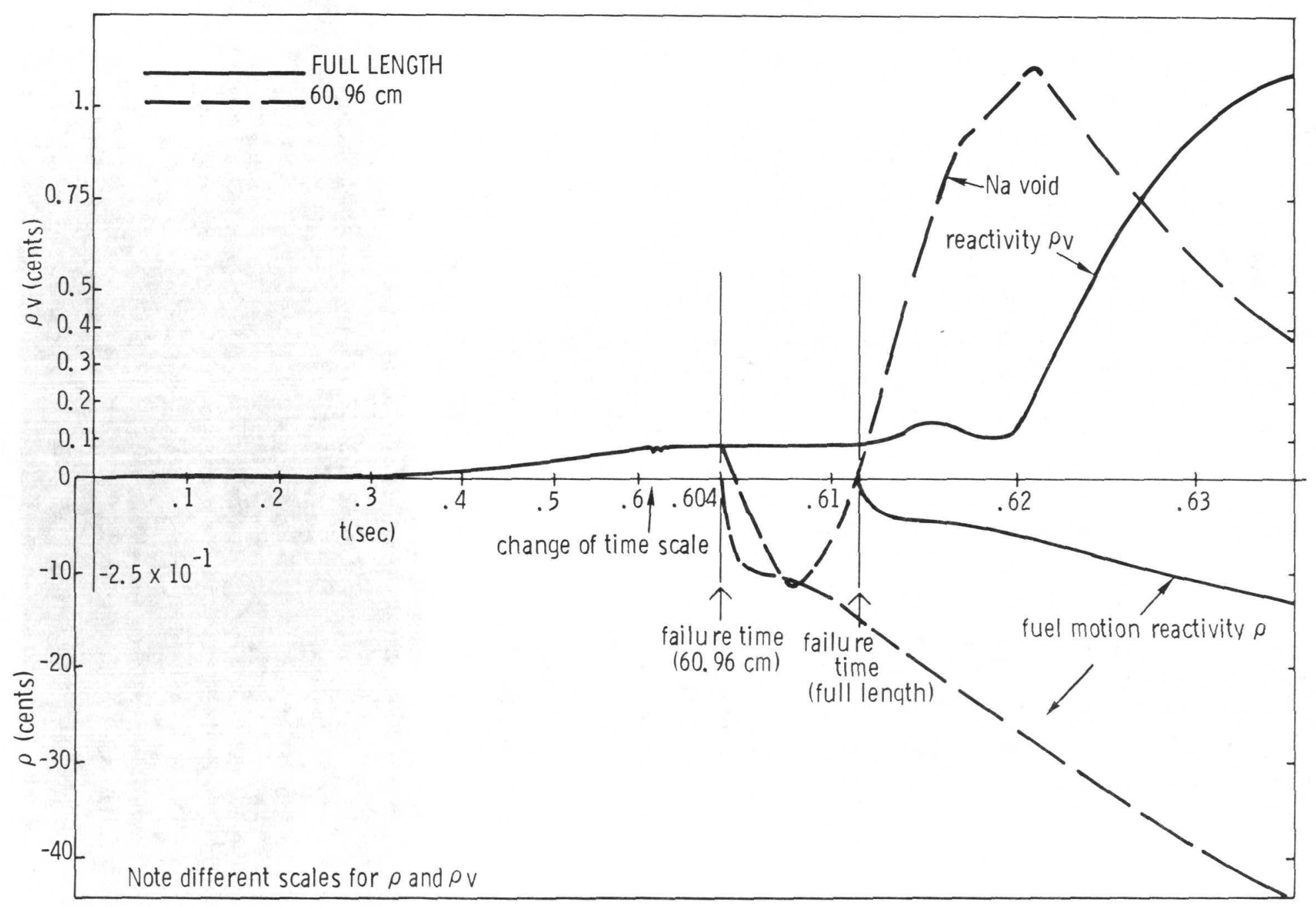

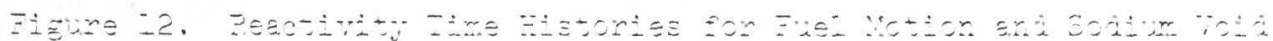


limit is $1250^{\circ} \mathrm{C}, C_{D} \leq 0.01$ for $2500 \mathrm{~J} / \mathrm{g}$ in prototypic test fuel and $\leqslant 0.022$ for $5000 \mathrm{~J} / \mathrm{g}$ in fully enriched test fuel. Low BeO-UO 2 ratios and enrichments are required to meet these criteria and the spectra requirements simultaneously. An infinite media parametric study of $\mathrm{BeO}-\mathrm{UO}_{2}$ fuels for gas-cooled systems is shown in Figure 13. Potential fuels must satisfy the $C_{D}$ criteria stated above and produce spectra that peak in the $100-400 \mathrm{keV}$ energy group and be reactive enough to have a $k_{\infty}>1.35$. Generally, the spectra criteria will be satisfied if the $k_{\infty}$ criteria are met and if the $\mathrm{BeO}-\mathrm{UO}_{2}$ ratio is < $20 / 1$. The reactor must have kinetic properties and material properties to allow pulse periods down to $1 \mathrm{~ms}$ and pulse widths of $5 \mathrm{~ms}$.

Calculations on $\mathrm{BeO}-\mathrm{UO}_{2}$ water-cooled systems were also initiated. A narrow convertor region of $\mathrm{UO}_{2}$ in iron was able to reduce peaking in a $93 \%$ enriched test subassembly to $\sim 1.8$. Further reductions are possible by enlarging the convertor region and by replacing water in the convertor region with a gas coolant.

Software has been developed to generate adiabatic temperatures in test fuel and driver fuels as a final edit option in the DTF-IV neutron transport code ${ }^{20}$ and to prepare input data on physical properties of the fuel materials. Some preliminary work leading towards the creation of temperature dependent cross section libraries has also been done using the AMPX system from oak Ridge. ${ }^{21}$

Proposed modifications to the Sandia Engineering Reactor (SER) have been reviewed in light of the anticipated experimental requirements for HFFPR. A preliminary cost estimate for these modifications to the plant and reactor are given in Table III.

\subsubsection{Large-Scale Tests}

One of the main problems facing the U. S. LMFBR Safety Program is a lack of adequate facilities to do large-scale testing. New facilities and upgrades of existing facilities have been proposed, but there is a possibility that they will not be available in time to support a 1986 commercialization decision. The Nation's Underground Nuclear Test Technology Program, which utilizes the facilities at the Nevada Test Site, has the potential for providing some of these needed data from 5 to 7 years before the proposed safety test facilities will be on-line. Studies are currently under way to determine the feasibility of such tests. Included in the study are source-driven, self-driven, and hybrid tests. 


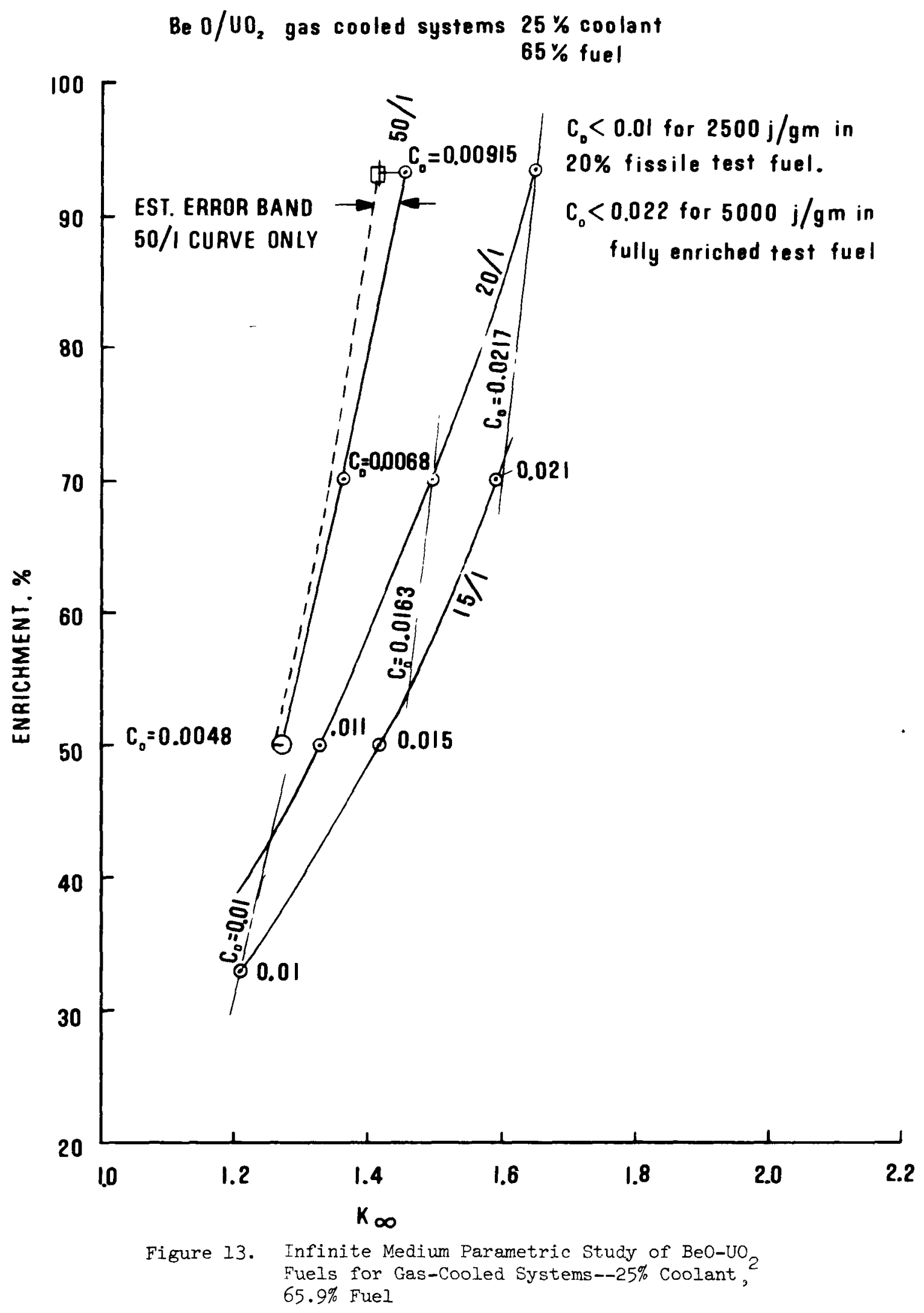


TABLE III.

Preliminary Cost Estimates for the Modification of SER to Satisfy HFFPR Experimental Requirements

Water Cooled
\begin{tabular}{ll} 
Building Modifications & $\$ 3.08 \mathrm{M}$ \\
Reactor & $17.05 \mathrm{M}$ \\
\hline
\end{tabular}

Total

Gas Cooled

\begin{tabular}{ll} 
Building Modifications & $\$ 6.88 \mathrm{M}$ \\
Reactor & $18.10 \mathrm{M}$ \\
\hline
\end{tabular}

Total
$\$ 20.13 \mathrm{M}$

$\$ 24.98 \mathrm{M}$ 
Studies were initiated in October 1976 to investigate the design of an Underground Test Facility (UGTF) utilizing the Nevada Test Site. These tests would address prompt burst excursions (PBE), transition phase recriticality, postaccident heat removal, radiological source term evaluation and other issues requiring a data base from large-scale tests. The reference designs and requirements for an external source driven subprompt critical assembly (ESDA) and a self-driven assembly (SDA) are described in the Fourth Quarterly of 1976. Work is continuing on these designs and some new concepts have been initiated as well. External sources that have been considered include both nuclear devices and thermal reactors. During this quarter, our emphasis has been on coupled core systems.

1.6.2.I Design Results - Design calculations were performed using a coupled point kinetics/heat transfer code to examine the pulse characteristics and target energy depositions achievable with an ESDA concept. The design target energy deposition of $1000 \mathrm{cal} / \mathrm{g}$ is achievable for an assembly with a figure of merit of 7 , Doppler thermal feedback of $1.4 \times 10^{-5} \Delta \rho /{ }^{\circ} \mathrm{C}$, and a reactivity of approximately 0.35 dollars (Figure 14).

A significant safety advantage is recognized in the ESDA concept as opposed to previously considered large-scale test facilities. Since the target fuel can insert large amounts of reactivity as a result of fuel motion, recriticality is a crucial problem. The problem is intensified because a fast neutron environment and short pulse in the target region is necessary for prototypic test conditions which work against inherent shutdown from thermal feedback. Previous facility designs have incorporated multi-region core grading with a large thermal spectrum driver region to provide the required shutdown. Not only is this concept very expensive, but there is an inevitable dependence on complex control systems. This dilemma is avoided in the ESDA due to the nature of the reactivity insertion required for the pulse and is totally removed from the system at the end of the external pulse. The inherent shutdown available in the system is given as

$$
\text { Shutdown }=(\text { Doppler shutdown })-(\text { reactivity of core })-(\text { compaction reactivity }) .
$$

The Doppler in the case described in Figure 14, in which the neutron source pulse is from a thermal reactor such as the ACPR, is \$3.18; the necessary reactivity for $1000 \mathrm{cal} / \mathrm{g}$ with a figure of merit of 7 is $\$ 0.35$; and the maximum credible compaction is $\$ 2.00 ;{ }^{22}$ thus, the shutdown is $\$ 0.83$. 


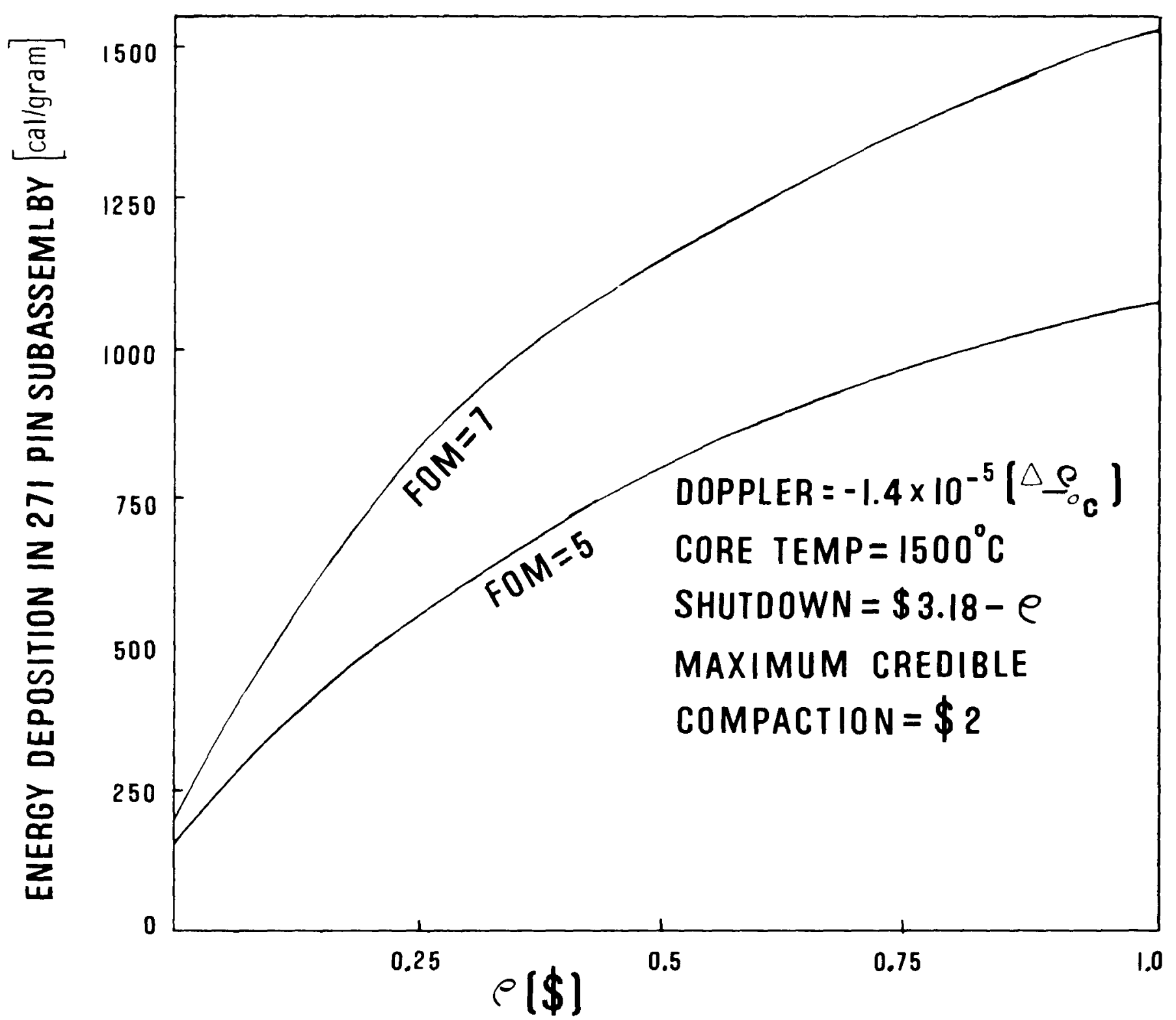




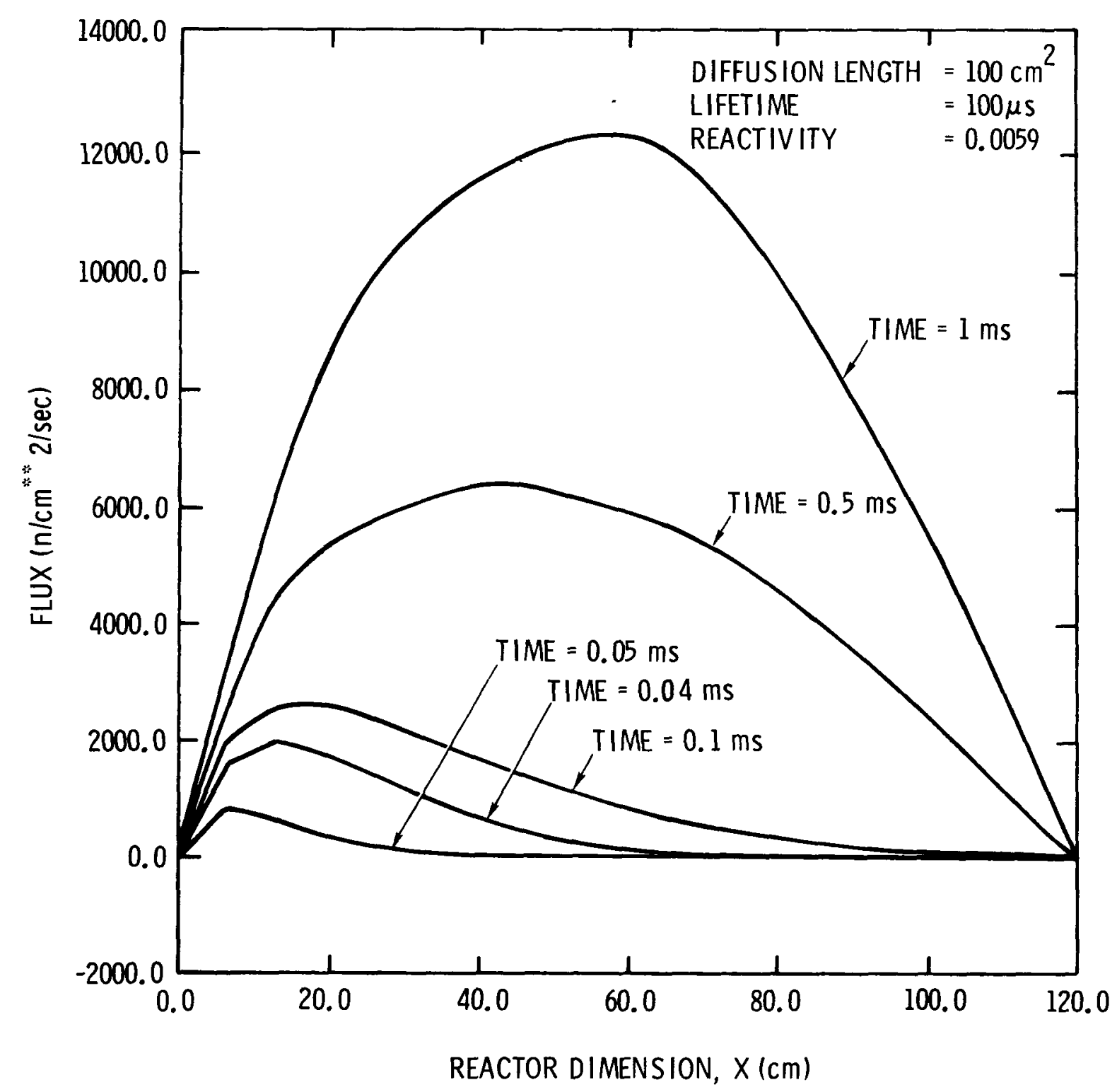

Figure 15. Spatial Flux versus Time for Pulse Source ( Time $=5.000 \mathrm{E}-05$ to $1.000 \mathrm{E}+00 \mathrm{~ms}$ ) 
The validity of the conclusions drawn from point kinetics calculations depends on the quantification of source effectiveness; that is, the number of fissions induced per source neutron. Since the source pulse is very spatially dependent, the attainment of fundamental mode in the assembly is of interest. Calculations were performed determining the time dependence of the spatial flux distribution. The results displayed in Figure 15, demonstrate that fundamental mode is achieved in short enought time to justify the point kinetics treatment utilized.

1.6.2.2 Coupled-Core Concept - Studies have been initiated to examine the possibility of providing the external source to the ESDA with a pulsed thermal reactor core. Clear safety advantages exist in such a system as a result of the low reactivity in the fast portion of the system. Further, the pulse characteristics would be dominated by the thermal core, thus, providing flexible test characteristics.

The coupled core concept is being studied with a coupled space-time, heat transfer code in order to verify the theoretical model used for scoping and results will be presented in the next quarterly report. 
0

- 


\section{CORE DEBRIS BEHAVIOR}

\subsection{Molten Core Technology}

(F. E. Arellano, 5831; D. A. Dahlgren, 5411;

N. R. Keltner, 9337; R. I. Knight, 5411, D. A. Powers, 5831;

A. F. Turbett, 5831; F. Zanner, 5833; T. Y. Chu, 9337)

\subsubsection{Introduction}

In the analysis of LMFBR core disruptive accidents, a number of scenarios lead to the release of molten core material from the guard vessel. This molten material is primarily steel and uranium dioxide plus the radioactive by-products. The molten material enters the reactor cavity and either directly contacts the cavity boundary or fragments upon contact with sodium which might be in the cavity. In the latter case, the debris bed formed could dry out, resulting in molten material contacting the cavity boundary.

The reactor cavity boundary can consist of anything between bare concrete and a fully designed core catcher. Because the near-term program emphasis is on timely results for use by $\mathrm{NRC/NRR}$ in the evaluation of CRBR, the study is currentIy aimed at cavity boundary designs important in the inherent retention concept. The program is designed to explore other concepts in the future utilizing the technology developed in the near term.

The inherent retention concept is based on a steel-lined concrete structure, possibly including a firebrick insulating material. The first evaluation for the inherent retention concept should consider the retention capability of the concrete alone. Following this, the interaction with the steel liner should be evaluated along with the insulating material. The phenomena of importance include penetration rates, gas evolution species and rates, and material solubility.

At the request of NRC/NRR, the initial program definition has been modified to include a large-scale transient molten steel/CRBR concrete interaction and four 75-pound "thermitically" generated melt/concrete interactions. 


\subsubsection{High-Temperature Melt-Liner Interactions}

Four tests of the interactions between high-temperature, thermitically generated, steel melts and 3/8-inch thick steel liner plates were conducted. Variables in the tests were intended to alter the velocity of the melt when it impacts the liner. Summaries of the test results are provided in Table IV. Velocities reported in this table are average velocities, and may be altered as analysis of the experimental results proceeds. Results of plate test 7 , in particular, may be in considerable error.

Table IV

Results of Tests Involving High-Temperature, Melt-Liner Interactions

\begin{tabular}{ccc} 
Test & $\begin{array}{c}\text { Time of Penetration } \\
(\mathrm{s})\end{array}$ & $\begin{array}{c}\text { Velocity of Melt } \\
(\mathrm{cm} / \mathrm{s})\end{array}$ \\
\cline { 2 - 3 } & 1.290 & 156 \\
Plate 5 & 1.033 & 232 \\
Plate 6 & 0.586 & 186 \\
Plate 7 & 0.650 & 220 \\
Plate 8 & &
\end{tabular}

\subsubsection{Transient High-Temperature Melt-Concrete Interactions}

Design of the instrumentation tower for the four experiments planned in this phase of the experimental program has been experimentally confirmed. The design basis of the instrumentation tower is to place sufficient passive cooling masses in the pathway of gas streams exiting the site of melt-concrete interactions to prevent excessive heat loads on either the tower structure or the associated instrumentation. The instrumentation which will be built into the tower includes gas evolution monitors, gas samplers, aerosol samplers and gas temperature monitors.

\subsubsection{Large-Scale Molten Uranium Dioxide - Concrete Interactions}

Discussions were held with personnel from Cheston, Inc., Inductotherm, Inc., and Super-Temp, Inc., concerning modification of the furnace and associated electrics 
for the formation and deposition of large ( $>100 \mathrm{~kg}$ ) uranium dioxide melts. Preliminary design bases revolved around a hot zone $61.9 \mathrm{~cm}$ high and $41.4 \mathrm{~cm}$ in diameter. Heating will be provided by induction on a carbon susceptor. Furnace designs will allow the melt to be deposited into a cold crucible.

Complete specifications and requests-for-quote are being prepared and will be forwarded to the companies named above.

\subsubsection{Large-Scale, Sustained Molten Steel - Concrete Interactions}

Fabrication of devices to allow gases generated during molten steel - concrete interactions past sensors has begun. These devices will cap the concrete crucibles described previous $1 y^{23}$ after molten steel has been deposited. Schematic diagrams of the devices are shown in Figure 16. In essence, the device is a cylindrical stack which will be sealed to the crucible after metal has been teemed in.

\subsubsection{Modeling and Analyses}

A model has been formulated to predict the relesse and migration of water, water vapor and carbon dioxide in concrete. Heat flow in the medium is calculated from

$$
\rho C_{P} \frac{\partial U}{\partial t}=\nabla \cdot(\chi \nabla U)-\rho_{W} V_{I} \cdot \nabla\left(U C_{P W}\right)+\dot{Q}
$$

The second term on the right accounts for convective heat flow due to water motion. The seepage velocity, $S_{i}$, of species $i$, is determined by Darcy's Law

$$
\rho_{i} V_{i}=-\lambda_{i} V P \quad i=1,2,3 .
$$

The distribution of the fluids in the concrete is obtained from the equations of continuity for each species

$$
\frac{\partial \omega_{i}}{\partial t}=-V \cdot\left(F_{i k}+F_{i m}\right)+S_{i} \quad i=1,2,3 .
$$

in which $F_{i k}$ is the flux due to kinetic motion, $F_{i m}$ is the molecular diffusion flus, $s_{i}$ is a source term for species $i$, and where $w_{i}$ is the volumetric concentration of species $i$, defined by

$$
w_{i}=\frac{m_{i}}{V} .
$$




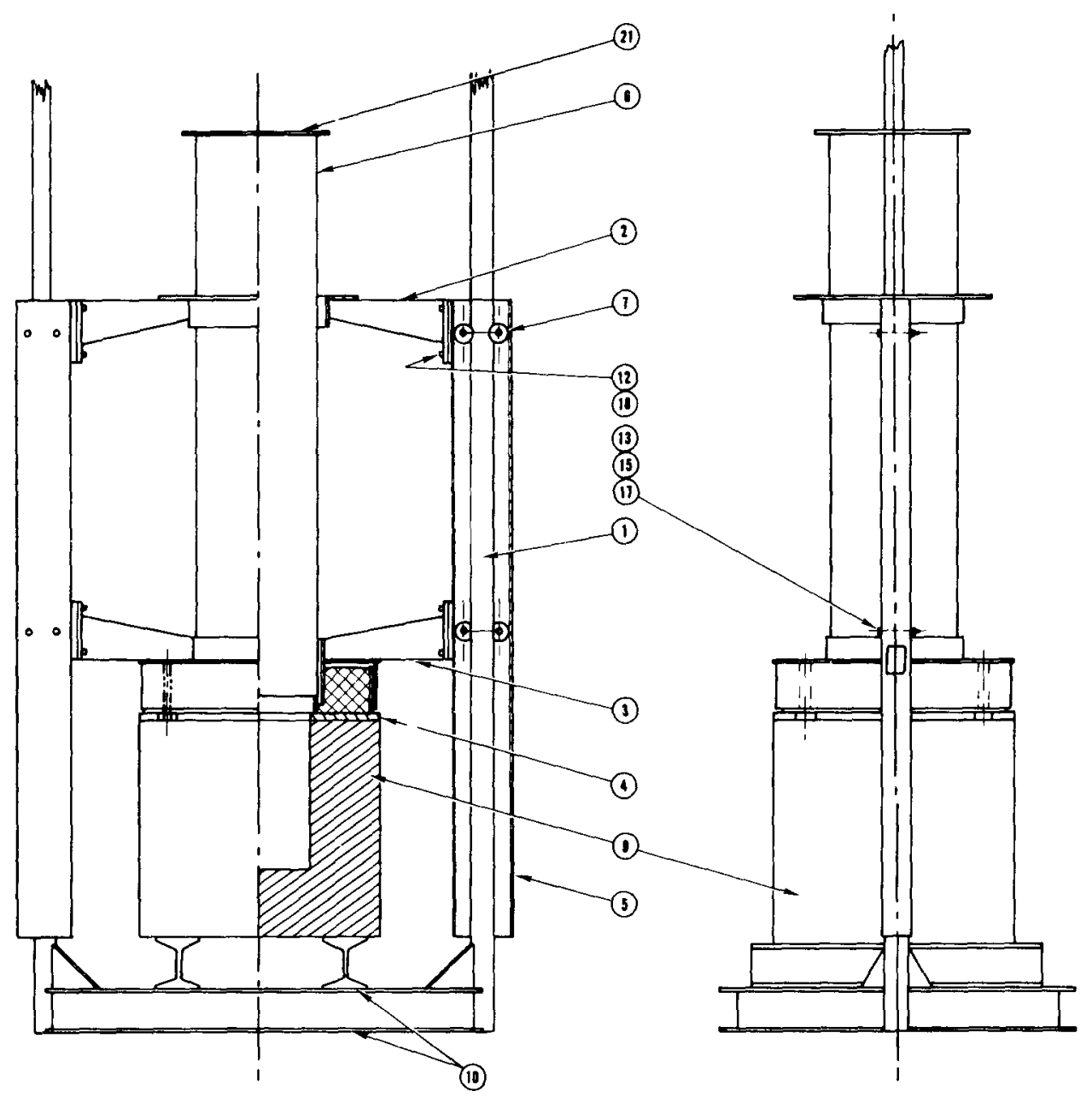

\begin{tabular}{|c|c|c|c|}
\hline TTEM & IDENTIFICATION & DESCRIPTION & $\begin{array}{l}\text { NO } \\
\text { REQD }\end{array}$ \\
\hline 21 & & GASKET, HI. TEMP & 1 \\
\hline \multicolumn{4}{|l|}{20} \\
\hline \multicolumn{4}{|l|}{19} \\
\hline 18 & & WASHER, LOCK, $1 / 2$ & 16 \\
\hline 17 & & WASHER, FLAT, I/2 & 8 \\
\hline \multicolumn{4}{|l|}{16} \\
\hline 15 & & NUT $1 / 2-13$ ESNA & 8 \\
\hline \multicolumn{4}{|l|}{14} \\
\hline 13 & & BOLT $1 / 2^{-13} \times 5 \mathrm{IN} . \mathrm{LG}$ & 8 \\
\hline 12 & & BOLT $1 / 2^{-13} \times 1 \mathrm{IN}, \mathrm{LG}$. & 16 \\
\hline \multicolumn{4}{|l|}{11} \\
\hline 10 & & $6 \mathrm{IN}$. STEEL 1 BEAM $\times 36 \mathrm{LG}$. & 2 \\
\hline 9 & & CRUCIBLE & 1 \\
\hline \multicolumn{4}{|l|}{8} \\
\hline 7 & KTC-S9-77006-3 & ROLLER & 8 \\
\hline 6 & KTC-S9-77006-2 & SIEEVE & 1 \\
\hline 5 & KTC-59-77006-1 & SIDE POST & 2 \\
\hline 4 & KTC-59-77005 & CRUCIBLE TOP PLATE & 1 \\
\hline 3 & KTC - $59-77004$ & LOWER SUPPORT & 1 \\
\hline 2 & KTC-S9-77003 & UPPER SUPPORT & 1 \\
\hline 1 & KTC-S9-77002 & GUIDE RAIL & I \\
\hline
\end{tabular}

Figure 16. Top Hat Assembly 
Here $m_{i}$ is the fluid mass and $V$ is the bulk volume of the porous medium including matrix and pore space. The consensus of current literature is that $F_{i k}$ is large compared to $\mathrm{F}_{i m}$ for fluid motion in concrete.

After determining the mass distribution, the partial pressure of each species is determined from the equation of state of that species. Presently, the ideal gas equation is being used.

The release of $\mathrm{H}_{2} \mathrm{O}$ and $\mathrm{CO}_{2}$ is determined from empirical relations derived by Dana Powers. 23 The rate equations are of the form

$$
\frac{d \alpha_{i}}{d t}=K_{0}\left(1-\alpha_{i}\right) e^{-(A / R T)} \quad i=1,2,3
$$

where $K_{0}$ and $A$ are experimentally determined parameters and where $\alpha_{i}$ is the fraction of reactant decomposed. In this equation, the three values of $i$ represent evaporable water, chemically constituted water, and carbon dioxide. The fluids released contribute to the source terms in the continuity equations. In the temperature range $500^{\circ}-1100^{\circ} \mathrm{C}$, the $\mathrm{CO}_{2}$ release is important because its large quantity can dominate in the pressure calculation.

The above equations, together with Dalton's Law, are solved simultaneously to obtain temperature and pressure, in addition to the concentration and velocity of each species at each mesh point in the concrete.

\subsection{Debris Bed Studies}

(J. B. Rivard, 5422; J. A. Brammer, 1136; A. R. Phillips, 5423; D. N. Cox, 5422;

H. G. Plein, 5422; H. C. Hardee, 1262; R. H. Nilson, 1262; D. 0. Lee, 1262; P. W. Conrad, 54II)

\subsubsection{Introduction}

The objective of the experimental studies of LMFBR debris beds is to provide information on the behavior of reactor materials following a hypothetical core disruptive accident. Initially, it is desired to characterize the sodium dryout instability in a bed of frozen fuel particulate which may lead to fuel remelt and associated high temperatures in a typical IMFBR. 
Activity during the report period was centered on assembly and testing of the apparatus required for the initial in-pile experiment series ( $D-1, D-2$ and $D-3$ ).

\subsubsection{Experiment}

On December 3, 1976, the out-of-pile systems test (OPST) capsule was filled with $2.24 \mathrm{~kg}$ of sodium in preparation for the OPST. The principal objective of the OPST is to verify the thermal performance of the debris bed experiment package and the supporting helium cooling loop. For the test, the experiment capsule contains an electrical heater assembly in place of the debris bed canister. This heater can deliver up to about $10 \mathrm{~kW}$ of heater power to the sodium in which it is immersed, simulating the in-pile heating of fuel. The experiment package is assembled into a 25-foot deep hole at the outdoor test station where it is connected to the helium cooling loop.

Prior to the filling of the OPST capsule, it was determined that the capsule room temperature leak rate was on the order of $10^{-7} \mathrm{standard} \mathrm{cc} / \mathrm{s}$.

During the following 1-1/2 months, the experiment package was assembled at the outdoor test site and mated to the helium cooling loop. Delays were experienced caused by repairs of damaged and faulty apparatus which produced leaks in the helium vessels and seals, and also by the plant shutdown over the Christmas/New Year holidays.

On January 24,1977 , the system was operated for the first time at rated helium pressure; on January 27, the sodium in the capsule was melted and a controlled sodium temperature of $673 \mathrm{~K}\left(400^{\circ} \mathrm{C}\right)$ was attained with a capsule heater power of $4 \mathrm{~kW}$ simulating an operating debris bed. Subsequent testing has established preliminary characteristics of the system which include sodium temperature control witrin a few degrees $\mathrm{C}$ at steady conditions, achievement of steady power diagnostics within 30 minutes following a large change in temperature or power, helium flow rates and pressure drops which closely match design values, and helium heat rejection capacity equal to or greater than the design value. At this writing, the system has been operated with a maximum load of $9 \mathrm{~kW}$ and at a maximum temperature of $873 \mathrm{~K}\left(600^{\circ} \mathrm{C}\right)$. Testing is continuing to establish the following: 
a. General operating characteristics of the system under various operating loads and temperatures.

b. Helium flow controller settings to optimize the transient response of the control function.

c. Optimum choices in data acquisition modes and digital processing for the power diagnostic.

d. Confirmation of the correct operation of safety-related control and instrumentation functions.

Following the OPST, the reactor compatibility experiment ( $R C E$ ) will be performed. For the RCE, the entire experiment package will be assembled and loaded into the ACPR central irradiation cavity with the exception that no fuel $\left(\mathrm{UO}_{2}\right)$ or sodium will be included. The helium piping to the helium cooling loop up to the door penetration will be connected and a helium leak check will be performed on the experiment system. A reactivity worth measurement will be made, shielding efficiency will be determined, and the influence of the experiment on reactor operation will be assessed. Dosimetry data will be obtained. The experiment package will be removed from the reactor using the techniques and equipment developed for handling the irradiated experiment to test the practicability of the methods.

\subsubsection{Studies and Analysis}

Out-of-pile studies of single- and two-phase convection in internally heated porous media are continuing in support of the debris bed experimentation. A principal finding is that the amount of subcooling of the bulk fluid (above the bed) has a significant influence on boiling beds, since subcooling allows condensation of vapor within the bed, thereby mitigating the disruptive effects which characterize upheaval and channeling.

In steady-state boiling convection, three distinct regions of the bed are identified:

a. The vapor dominated boiling region is in the bottom of the bed where the temperature is uniform and latent heat transport removes all of the energy input.

(1) The two-phase flow pattern is not a cellular concurrent flow, but involves liquid downflow and vapor upflow in close proximity all across the bed. 
(2) The boiling region does not enlarge significantly as the power is increased. A vapor dominated dome forms at the onset of boiling which then flattens until the upper boundary is nearly horizontal, but upward progress with increasing power is not observed.

b. The condensation zone is a narrow band in the middle of the bed where rising vapor is condensed. At moderate and intermediate boiling rates, the liquid resupply to the boiling region is largely due to the recirculation of condensate.

c. The liquid dominated region in the upper part of the bed changes considerably in character as the energy input is increased.

(1) At low power levels just beyond the boiling transition, there is no vapor penetration above the condensation band. Single-phase cellular convection of the liquid is observed, but conduction dominates.

(2) At intermediate power levels, vapor does penetrate into the upper region. Pockets are formed which sporadically release vapor to the surface and are then reflooded with liquid.

(3) At high power levels, channels open which connect the boiling region to the surface, allowing direct and nearly continuous venting of vapor. The upper region is now fully wetted with the exception of the channels. Imbedded vapor pockets are no longer present.

Disruptive phenomena, such as channeling and upheaval, are very important but not well understood. The present experiments illustrate the following features:

a. Subcooling of the overlying pool allows condensation within the bed, thereby increasing the power which is required to induce disruption.

b. The cohesiveness of the particulate (determined by size, shape, roughness, etc.) has a major influence on the occurrence of disruptions. For example, the upheavals and deep channeling observed in sand beds do not occur in beds of glass beads. 
The disruptive mechanism is also related to the upward progress of discrete vapor bubbles through the porous matrix and the formation of stalled vapor pockets.

\subsubsection{Status}

The out-of-pile sytems test (OPST) is in progress and will be completed shortly, followed by the RCE and the first in-pile experiment. Out-of-pile studies are continuing to build basic understanding of debris bed phenomenology.

\subsection{Molten Fuel Pool Studies}

(H. G. Plein, 5422; G. A. Carlson, 5423;

W. H. Sullivan, 5423; and M. R. Goebel, 1136)

\subsubsection{Introduction}

The primary objectives of the in-core molten fuel pool program include the investigation of the heat flux distribution and the container ablation potential of simulated LMFBR debris materials.

The major activities during this reporting period included the design, drafting and ordering of the containment vessels for the in-core experiments; an in-core qualification program for seals, pressure transducers and ultrasonic thermometers; and the drafting of the experiment plan to be submitted to the ACPR Committee.

\subsubsection{Experiment Design and Fabrication}

The fuel-only experiment capsule is shown in Figure 17. The design of the two containment vessels and the air flow shroud complete the package designs. The inner portions of the capsule have been described in an earlier report. ${ }^{8}$ The inner containment vessel will be 2.54-mm thick A-106 (or similar) steel. It is $194 \mathrm{~mm}$ in outside diameter and $284 \mathrm{~mm}$ in height. The outer containment vessel will be made of the same steel, is $216 \mathrm{~mm}$ in outside diameter, and is $398 \mathrm{~mm}$ in height. The bottoms of these vessels are $9.5-\mathrm{mm}$ thick steel to provide a thermal sink for solidifying molten fuel in case of a highly unlikely double crucible failure. The vessel lids will be $12.7 \mathrm{~mm}$ thick and will seal with Viton o-rings in the vessel. Threaded stainless steel feed-throughs will be welded into the lids to minimize leakage. A set of three eyebolts on each lid will be used to support or move the vessels. 


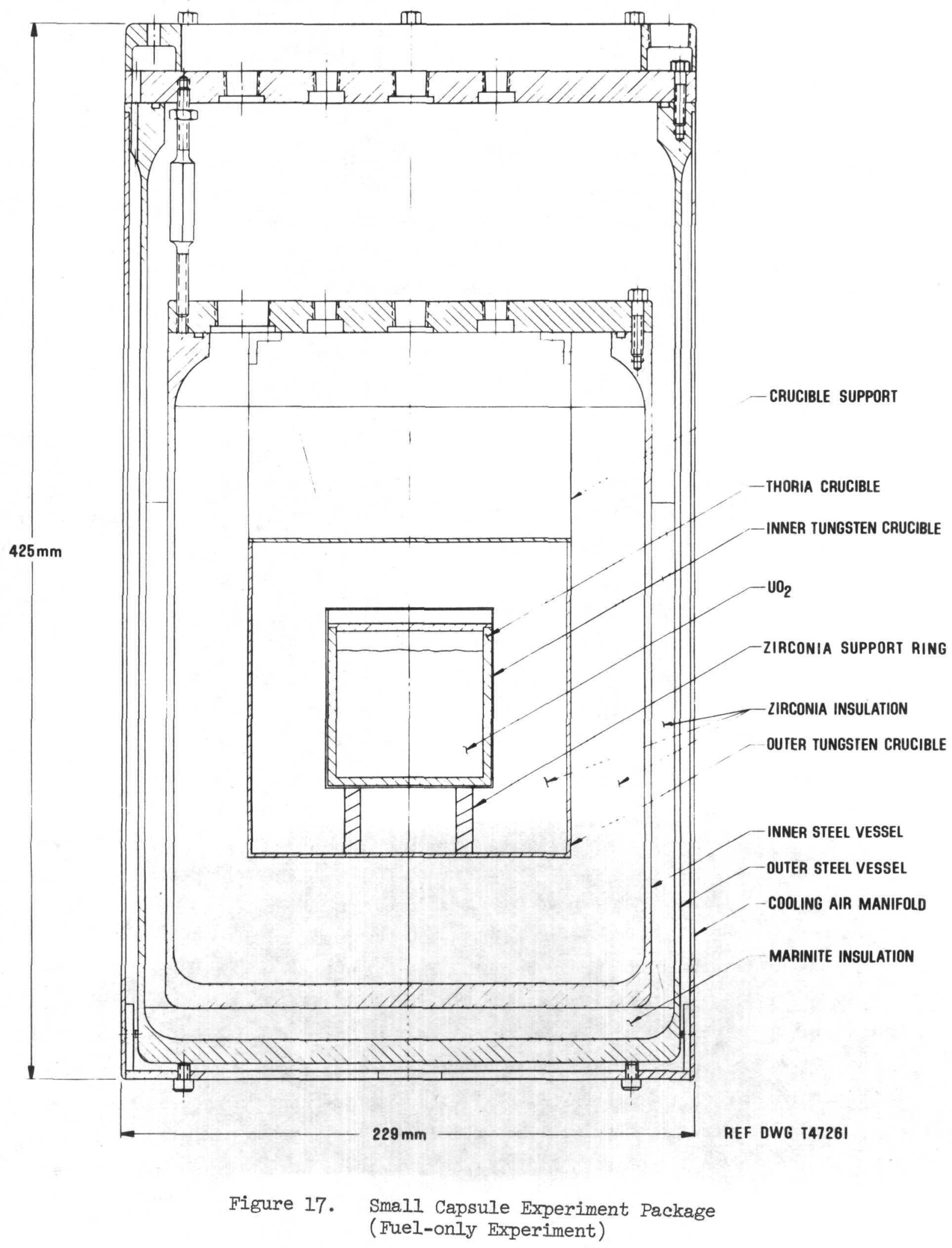


Outside the containment vessels, a 6061 aluminum air shroud will channel cooling air down and around the outer vessel to exit at the bottom center. The warmed air will be exhausted by the reactor cavity purge system.

The order has been placed for three each of the inner and outer containment vessels and lids, and cooling shrouds. Delivery is expected by mid-April. Either reuse of the vessels or an additional order will provide containment vessels for the completion of the small capsule series.

Materials for the in-core experiments continue to be received. Now on-hand are the continuous and multi-point recorders, signal-conditioning amplifiers, feed-throughs, thermocouples, valves and low temperature thermal insulation.

\subsubsection{Analysis}

Neutronics calculations were made with the two-dimensional code TWOTRAN 24 for the small capsule fuel-only experiment. The reactivity worth of the experiment capsule with $0.9 \mathrm{~kg} \mathrm{UO}{ }_{2}$ is about $-75 \phi$ in the ACPR. The worth of the fuel alone is only about $+19 \phi$ due to the large self-shielding effect of the enriched fuel.

Energy deposition within the fuel is highly peaked near the edges due to lack of thermal neutron filtering. Any filtering would decrease the overall deposition and lengthen the heating time to achieve melting. Heat transfer calculations indicate the temperature does not peak near the edges of the fuel.

Calculations of the pressure history within the inner containment vessel show the maximum pressure will be about three times that initially present. It is planned to fill the inner vessel with helium to about $40 \mathrm{kPa}(0.4 \mathrm{bar})$ prior to heating. Therefore, the maximum system pressure could get to $120 \mathrm{kPa}$ under normal conditions.

The pressue capability of the inner containment vessel at maximum temperatures is about $3.6 \mathrm{MPa}$. System pressure will be monitored to determine in-leakage and follow the system pressure history. 


\subsubsection{In-Core Component Qualification Experiments}

In an earlier experiment, Viton elastomeric seals were tested for radiation tolerance and found to be satisfactory for the molten pool experiments. This quarter, two more experiments have been performed to qualify seals, transducers, cabling, and electronic systems performance. In each experiment, a small aluminum pressure vessel, sealed with Viton o-rings and equipped with one or two pressure transducers, an ultrasonic thermometer, and a thermocouple, was irradiated in the core of the ACPR reactor at $300 \mathrm{~kW}$ power for 120 to 150 minutes. Active temperature and pressure measurements were taken throughout each experiment. In one experiment the vessel was pressurized to $200 \mathrm{kPa}$ using helium gas, and the vessel temperature was held at $373 \pm 5 \mathrm{~K}$. In the second experiment, the vessel pressure was cycled between 100 and $200 \mathrm{kPa}$ at 10 minute intervals, while the temperature was maintained for 60 minutes at $333 \mathrm{~K}$, then allowed to rise to $373 \mathrm{~K}$ for 90 minutes. The temperature and pressure conditions represent estimates of the conditions to be encountered by the pressure transducers during the molten pool experiments, while the total radiation dose is from 2 to 3 times that expected.

During these experiments, the thermocouple and ultrasonic thermometry diagnostics, all seals, and all cabling performed very well, with no significant radiation-induced damage evident in post-experiment examinations.

Further, the ultrasonic thermometry electronics recently developed performed quite well in this first reactor application. A technique of using the charged cable coil pulser at a 10-m distance from the pulse coil in order to provide isolation from the reactor core radiation was found to work well, requiring only some care to use relatively low inductance coils (< $30 \mu \mathrm{H}$ ) to minimize signal distortion.

Less satisfactory results were obtained with the pressure transducers. In each experiment, the semiconductor strain gauges tested began to show zero pressure shifts after 60-minute irradiation at $373 \mathrm{~K}$. The sensitivity of the gauges was unaffected by irradiation. There was some indication that operation at $333 \mathrm{~K}$ was less likely to result in significant zero pressure shifts than operation at $373 \mathrm{~K}$. 
Since the performance of these relatively low-cost transducers may be somewhat marginal, more expensive eddy-current type gauges will be used for critical pressure measurements in the early molten pool experiments. The semiconductor strain gauges will be included in these experiments for evaluation, but in less critical applications.

\subsubsection{Diagnostics Development}

The ultrasonic thermometry electronics modules for the in-core molten pool experiments are presently being fabricated. The data acquisition system for the initial experiments will be capable of analyzing signals from a five-sensor-element ultrasonic thermometer, and providing time interval data from each element to the DADS time-sharing computer. The system components have been evaluated with the cabling and interfacing required for reactor operation and found to operate very well. The jitter in time-interval data appears to be somewhat less than the $\pm 10 \mathrm{~ns}$ previously reported, apparently due to the relatively quiet electrical environment of the reactor area. Because the electronics system is being built with all functional units on different printed circuit boards, expansion to larger numbers of sensor elements or to additional ultrasonic thermometers will be relatively straightforward.

The diagnostics console for the small-capsule molten pool experiments has been designed, and most of the data acquisition electronics received (recorders, amplifiers, power supplies, etc.). Fabrication of the console has begun.

Because of the laser-welding difficulties, the small-diameter standoffs to be used for minimizing contact welding to the sheath have not yet been tested. Experience at EG\&G of Idaho with this standoff arrangement has been quite satisfactory. Some laser-welded standoffs have been obtained from EG\&G for evaluation. Further, EG\&G personnel have provided the laser-welder parameters they use to obtain satisfactory weld performance.

\subsubsection{Experiment Plan}

Before any experiment can be irradiated in the ACPR reactor, an experiment plan must be written for review and approval by the ACPR Committee. An experiment plan is now being written for the molten pool experiment. It includes a description 
of the apparatus and the diagnostics; analysis which has been done in support of the program (neutronics, heat transfer, thermodynamics, materials compatibility, and stress analysis); laboratory studies in support of the program (materials compatibility experiments, in-core component qualification, and nondestructive testing); the experiment schedule and experimental procedure; and safety considerations. The experiment plan currently being written will apply only to the fuel-only experiments, since the fuel-steel experiments require different crucible materials for containment. The fuel-steel experiment plan will be written and submitted after the initial fuel-only experiments have been conducted, allowing confirmatory data to be gathered on the performance of other parts of the system.

\subsubsection{Status}

Accomplishments this quarter include the completion of capsule design and the ordering of the final items for the in-core experiments. Several in-core qualification experiments were conducted for pressure seals, pressure transducers, and ultrasonic thermometers. The experiment plan was drafted for approval by the ACPR Committee.

Next quarter, the assembly and checkout of the in-core experiment capsule and diagnostics console will be conducted. The ultrasonic thermometry electronics modules now being assembled will be tested for use with the in-core experiments. The first fuel-only, small capsule, in-core experiment is scheduled for the last part of the quarter. 
(B. M. Butcher, 5167; D. A. Dahlgren, 5411; N. R. Keltner, 9337;

D. I. King, 9337; R. A. Sallach, 5831; R. L. Knight, 5411;

C. M. Stone, 543I; J. E. Smaardyk, 5167; H. J. Sutherland, 5167)

\subsection{Introduction}

Any loop-type IMFBR system must consider the interaction of hot sodium with cell liners and, given either a failed liner or a core disruptive accident, the interaction of hot sodium with concrete. The data base available for safety assessments involving these interactions is limited, especially for the concrete and failed liner interactions.

This program has been specifically developed to provide the research required to verify the data base from a confirmatory point of view. The initial sodiumconcrete interaction emphasis concerns:

a. Depth of penetration of sodium-concrete reactions.

b. Effect of sodium spills and sprays at various temperatures.

c. Data on water release, hydrogen evolution, and release of other products.

d. Determination of the extent of thermal cracking and exfoliation.

e. Effect of steel reinforcement on sodium-concrete reactions.

f. Determination of the reaction products.

The sodium/steel liner interactions will be studied for phenomenological identification and for explicit liner designs. The primary purpose of these experiments is to develop the consequences resulting from hot sodium spills or sprays on defective liners. The initial emphasis concerns:

a. The effect of defect size.

b. Determination of reaction products generated behind the liner plate, as well as gas pressures and temperatures.

c. Determination of the effectiveness of the vent system behind the liners in removing hydrogen and water vapor released from the concrete.

Iiner distortions will be studied as well as characterizing failure mechanisms. 


\subsection{Test Plan}

The test plan for the first series of large-scale sodium-concrete interaction experiments has been written. A draft of the plan has been distributed to test participants and NRC representatives for comments. The final report, incorporating these comments, will be issued during the next quarter.

The schedule for the first test series was agreed upon in the program review meeting with NRC representatives on March 8 and 9 . The first preliminary test will be conducted in early May with the first test of the instrumented series to take place in mid-June. Subsequent tests are planned at two to three week intervals.

\subsection{Concrete Crucibles}

The sixteen crucicibles for the test series have completed the required 90 days of cure and have been moved to the test site.

Table $V$ shows the slump, compressive strength, and ratio of water to cement and flyash for each pour. The slump and 90-day compressive strength are plotted against water to cement and flyash ratio on Figures 18 and 19. The straight lines on these graphs are the results of least square fits to the data. The low goodness of fit $\left(r^{2}\right)$ values are due to the inherent variability of concrete as well as the relatively small number of samples. Specification and design values are shown as dashed lines on these graphs. The design and specification values for strength are $5200 \mathrm{psi}$ and $4000 \mathrm{psi}$, respectively, at 28 days. The "Design" and "Spec." lines shown on Figure 19 are 25 percent higher than these values to account for the observed increased in strength after 90 days of cure. It appears from these data that the design mix should require a water/cement ratio of about .49 to .50 rather than the current .45 .

The prototypic insulated liner has been designed for the last test of the series. The liner consists of lightweight aggregate (crushed MgO), Perlite concrete, an Ethafoam spacer, and the steel liner, as shown in Figure 20. An existing crucible will be used for the structural concrete. The Perlite concrete will be cast early in the next quarter so it can be cured in time to e pport the test schedule. 
TABLE V

Slump, Strength, and Water/Cement Ratio for CRBRP Concrete Pours

$\begin{array}{rrllcc}\text { Pour } & \begin{array}{c}\text { Pour } \\ \text { Date }\end{array} & \underline{\text { W/C*}} & \begin{array}{l}\text { Slump } \\ \text { (Inch) }\end{array} & \begin{array}{c}\text { Day } \\ \text { Strength } \\ \text { (psi) }\end{array} & \begin{array}{c}90 \text { Day } \\ \text { Strength } \\ \text { (psi) }\end{array} \\ 1 & 8-30-76 & .51 & 5 & 5111 & 6721 \\ 2 & 8-30-76 & .48 & 3 & 5359 & 6208 \\ 3 & 8-30-76 & .48 & 2.5 & 6084 & 6845 \\ 4 & 8-30-76 & .48 & 2 & 4421 & 3608 * * \\ 5 & 10-05-76 & .48 & 2 & 6727 & 8357 \\ 6 & 10-13-76 & .53 & 5 & 3414 & 4762 \\ 7 & 10-22-76 & .50 & 3 & & 5386 \\ 8 & 10-22-76 & .50 & 3 & & 5147 \\ 9 & 11-03-76 & .50 & 3 & & 6226 \\ 10 & 11-05-76 & .50 & 2 & & 5704 \\ 11 & 11-16-76 & .52 & 3.5 & & 5704 \\ 12 & 11-16-76 & .53 & 5 & & 5978 \\ 13 & & .52 & 3.5 & & 5864 \\ 14 & 11-24-76 & .52 & 3.5 & & 5421 \\ 15 & 11-24-76 & .52 & 3 & & 6385 \\ 16 & 12-03-76 & .48 & 2 & & 5271 \\ 17 & 12-15-76 & .50 & 3 & & \end{array}$

* $\quad \mathrm{W} / \mathrm{C}=$ water $/$ cement + flyash

* Failed due to uneven loading of test cylinder. 


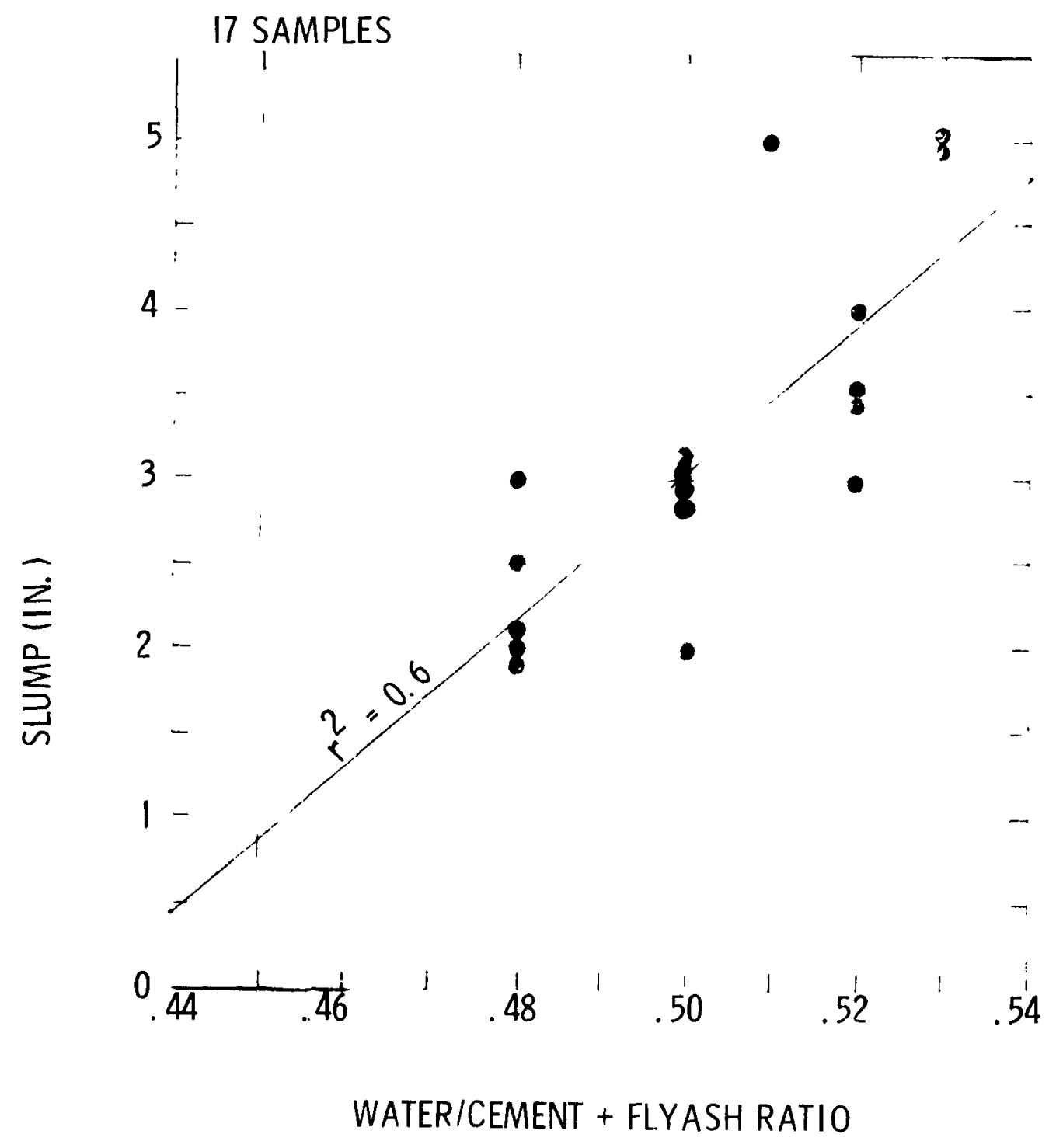

Figure 18. Slump and Water/Cement Ratio for CRBRP Concrete Pours 


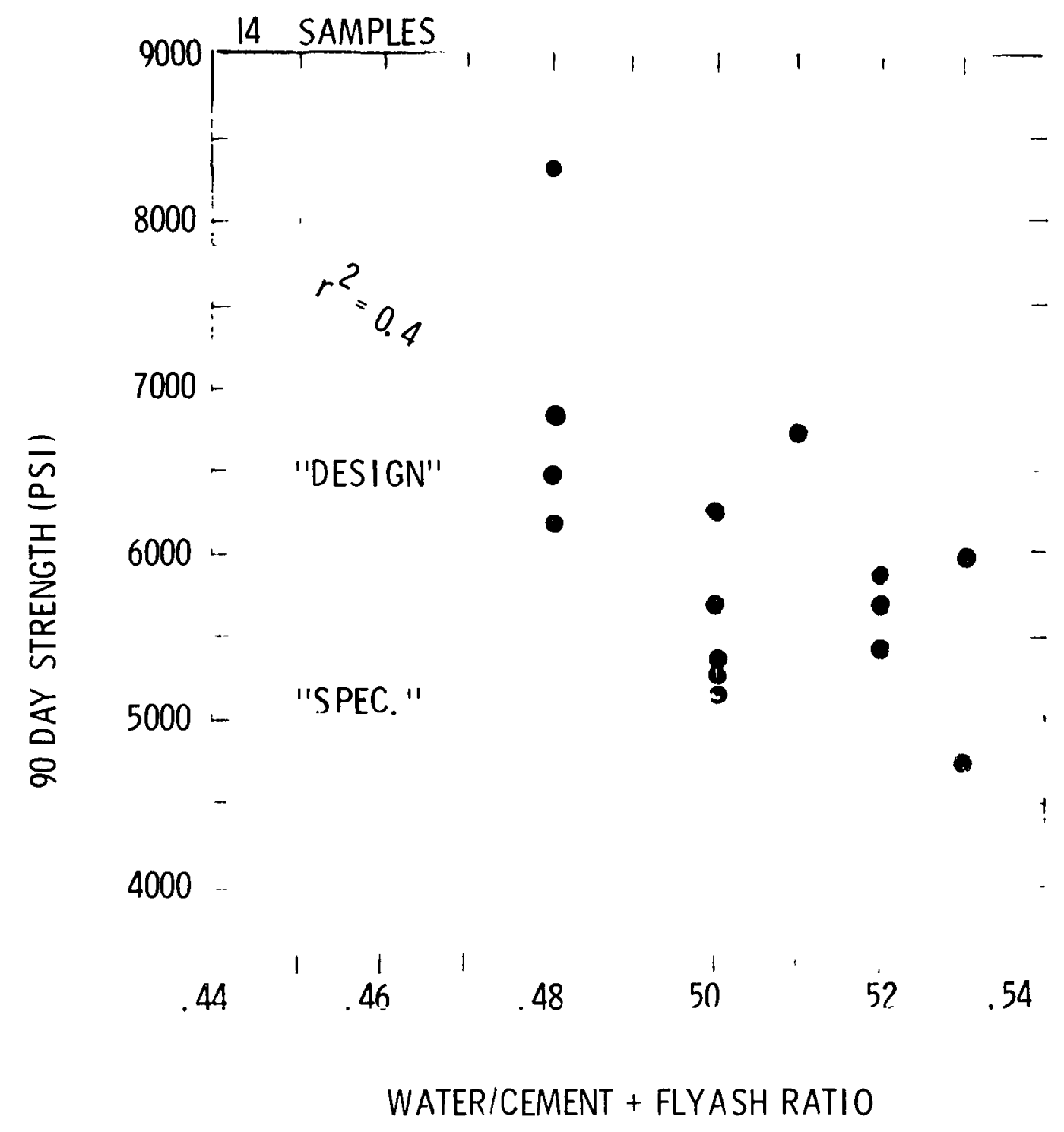

Figure 19. 90-Day Strength and Water/Cement Ratio for CRBRP Concrete Pours 


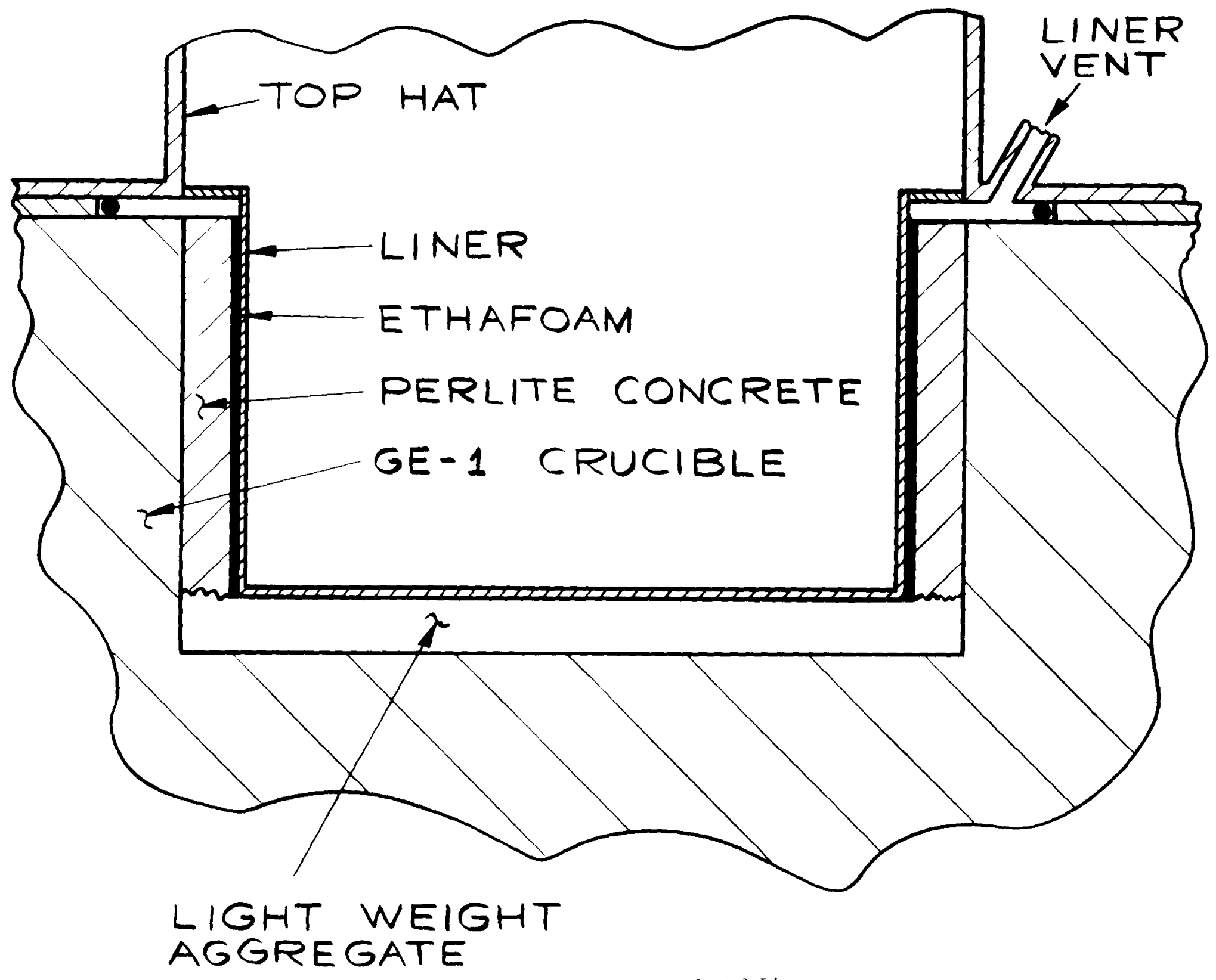

Figure 20. Prototypic Insulated Liner 
The Perlite aggregate for the insulated liner was obtained locally rather than from potential CRBRP sources as in the case of the concrete aggregate. This was based on information from the Perlite Institute, Inc., that the chemical and physical properties of Perlite are essentially independent of the source.

\subsection{Instrumentation}

The measurement of temperature, gas flow and composition, pressure, and reaction interface motion is essential for these experiments to gain a quantitative understanding of the sodium-concrete interaction. Table VI is a summary of the instrumentation to be used. The locations of the various sensors are shown in Figure 21.

The thermocouple measurements will be used to obtain in-depth temperature profiles. These temperature histories, initial data on the thermal conductivity of concrete as a function of temperature, and the acoustic penetration data will be used in a nonlinear, inverse heat conduction analysis to attempt to calculate the heat flux at the sodium/concrete interface. An attempt will be made to estimate the heat of reaction from the heat flux, the immersion heater power input, and the temperature at the reaction zone. The gas evolution rate and the ultrasonic penetration data can be used to determine the reaction rate. Finally, the heat flux, heat of reaction and the reaction rate estimates will be used to calculate the heat balance equation.

\subsection{Test Facility}

The present status of the sodium test facility is described in the following subsections.

\subsubsection{Test Chamber}

The test chamber structure itself has been situated and secured on a concrete $\mathrm{slab}$ on the west side of Fandia Puilding $9939 \mathrm{~A}$ (West Test Cell). Five Pesearch Incornorated 
I. Instrumentation embedded in the crucible

A. Thermocouples: 12

B. Moisture gages: 2

C. Ultrasonic transducers: 2 ( 1 transmitter, 1 receiver)

II. Instrumentation connected to the reaction chamber
A. Thermocouples: 6 ( 3 in sodium pool* and 3 in vapor space)
B. Pressure transducer: 1
C. Gas flow meter: $1^{* *}$
D. Gas analyzer: $1^{* *}$

III. Miscellaneous instrumentation

A. Load cells on sodium dump tank

B. Immersion heater (power input)

C. Catch tray to collect water that drains directly from the crucible

D. Television camera (located outside of viewing port in outer enclosure)

* One of these thermocouples will be placed behind the liner in the liner experiments.

** With automatic valving to monitor both chamber and liner vent lines. 


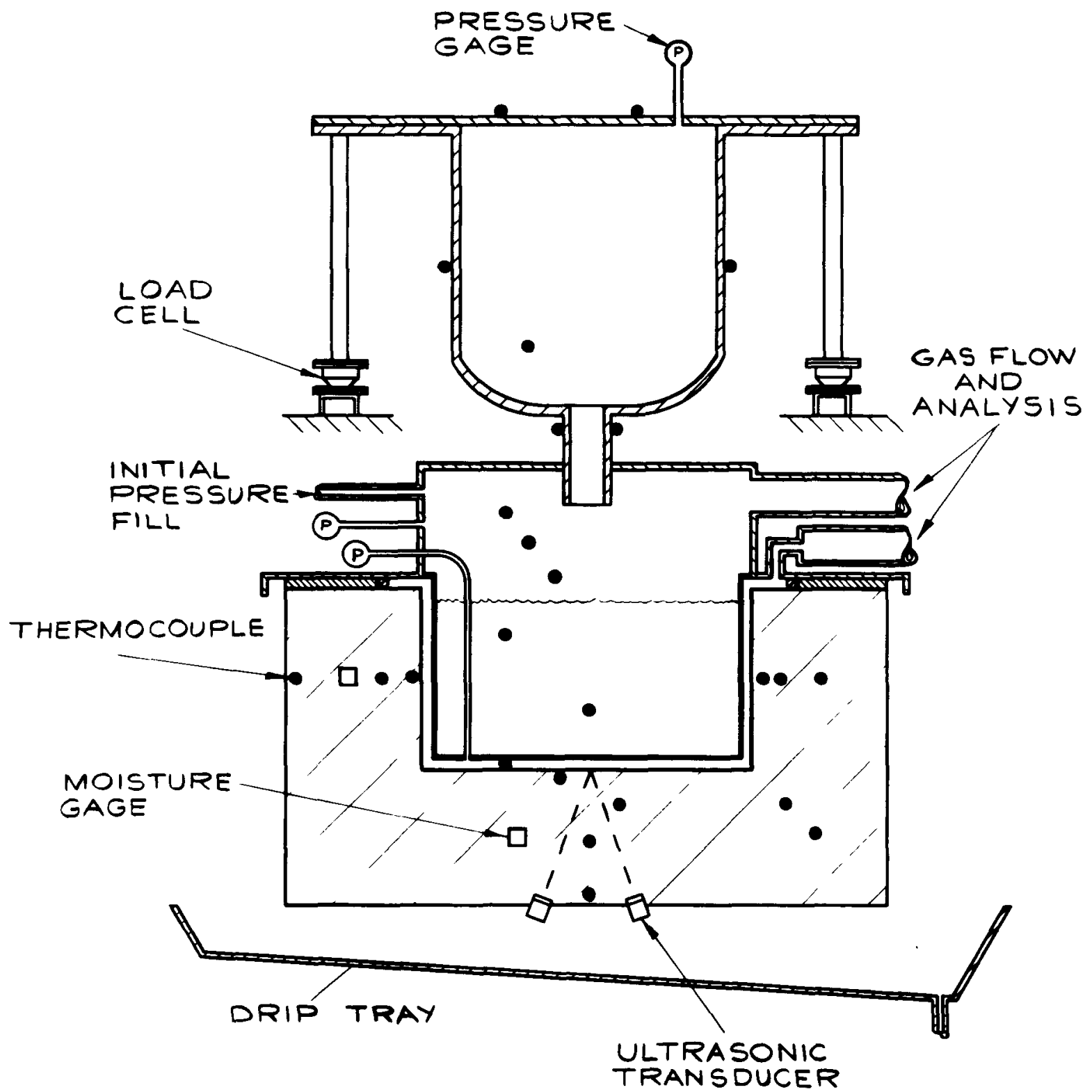

Figure 21. Location of Instrumentation 
Model 646 Dower controllers have been mounted to the test chamber and weatherproofed. Stairway access to the top of the test chamber as well as safety railings around the top have been installed. Cable trays, thermocouple extension wires, etc., are at the site ready to be installed. A steel stand to support the 55-gallon arum heater assembly has been fabricated and transported to the test site.

A potential scheduling problem was rectified with Sandia Plant Fnfineering and now the schedule completion date for the installation of power lines (to the power controllers) and inert gas supplies is March 31, 1977. Floodlights will also be installed to make 24-hour operation possible during the slow heating cycle of the sodium in the dump tank.

Telephones and intercoms are being installed in Buildings 9939 and $9939 \mathrm{~A}$ to facilitate remote control from Building 9939.

\subsubsection{Dump Tank}

All components and assemblies associated with the dump tank have been received and the final welding to be done on the tank will be completed on March 15, 1977. The heater shroud and tank support stand have been assembled and are ready for the installation of the dump tank. A support stand has been fabricated to enable transporting the tank from the welding shop to Sandia Laboratories, Area III, where the final assembly of the dump tank and the heater shroud will begin by March 18, 1977. After assembly the insulation will be applied and the tank can then be transported to the test site and assembled to the top of the test chamber on approximately March 31, 1977.

The load cells and support plates, pneumatic cylinder, solenoid actuated control valves, lubricator, etc., have been received and are ready for installation. 
The only components that are late from suppliers are the load cell indicator from Consolidated Controls Corp. and the stainless steel rupture disks to be used in the dump valve assembly. These items are expected March 15, 1977.

\subsubsection{Data Acquisition}

A sophisticated data acquisition and control system has been ordered consisting of a HP9825 calculator, NEFF620 high speed analog/digital multiplexer, HP6940 multiprogrammer, high speed analog recorders, computer compatible magnetic tape, digital $x-y$ plotter, and line printer. The majority of these items will be be functional for the preliminary sodium drops. For the preliminary tests, Honeywell multichannel strip chart recorders will be used to record load cell, pressure transducer, and thermocouple data.

\subsection{Gas and Posttest Chemical Analysis}

All gas analysis equipment has arrived and final calibration tests are in progress. Gas analyses will be made by two techniques. The more rapid is by a quadrupole mass spectrometer. For the first several tests, the mass spectrometer will continuously scan across the mass range I - 50 AMU at a repetition rate of once every two minutes to identify all possible evolved gas specie. In later tests, the mass spectrometer will be operated under control of the data acquisition system and only selected mass peaks will be monitored.

The above analyses will be backed up by other analyses by gas chromatography. The repetition rate with this technique is on the order of 10 minutes. Thus it provides mainly a periodic confirmation of the mass spectrometer, assuring that the latter's calibration remains valid.

The data on gas composition when combined with the pressure drop across the laminar flow element permits the calculation of flow rate. As the mass spectrometer comes under computer control, these calculations will be accomplished in real-time (concurrent with the test). 
Chemical analysis of the reaction products of molten sodium with the limestoneaggregate concrete will provide an insight into the major reaction processes. The following procedures were devised to treat a reaction product assumed to consist of sodium metal, sodium oxides (including hydroxide), sodium carbonate, calcium oxide, calcium carbonate, and graphite in various proportions.

Sampling will be accomplished by coring with steel tubes. From the cores, sectional samples will be taken to provide a depth profile.

The initial step is the conversion of the sodium metal into sodium bromide by means of a Wurtz reaction (reacting butyl bromide with sodium). The converted sample is then safe to handle using ordinary laboratory care. A portion of each sample will be examined by x-ray diffraction methods to determine the major components. The remainder of the sample will be then analyzed by conventional chemical procedures; the choice of procedure depending on the x-ray diffraction results. However, the following outline is generally indicated.

The water soluble components are to be checked for:

a. $\mathrm{Br}^{-}$content -- from which the original Na metal content can be calculated.

b. Alkalinity -- from which $\left(\mathrm{O}^{-}+\mathrm{OH}^{-}\right)$and $\mathrm{CO}_{3}=$ contents are obtained.

c. $\mathrm{Na}^{+}$and $\mathrm{Ca}^{++}$-- by atomic absorption methods.

The water insoluble material will be checked for the presence of graphite, undissolved $\mathrm{CaCO}_{3}$, and $\mathrm{SiO}_{2}$. Other components, if any, will be searched for and analyzed by appropriate procedures.

\subsection{CACECO Predictions}

The CACECO code has been set up to predict the first instrumented sodiumconcrete interaction experiment. The concrete crucible and its top hat have each been modeled as one-dimensional heat structures. 
Gas flow through the vent pipe from the top hat was treated in the code as a nozzle flow problem. This was achieved by calculating an effective nozzle coefficient for the pipe.

Water release from the heated concrete in the crucible is presently being predicted, based on experimental results of four HEDL concrete drying experiments. Preliminary work has been done to use the results of Dana Power's experimental work at Sandia to predict both water and carbon dioxide release. Powers has shown that both the water and concrete release from concrete depend not only on the temperature of the concrete, but also on the rate at which it is heated. Since the latter effect can account for several orders of magnitude in the results, it definitely should be incorporated into containment codes. CACECO only accounts for the temperature dependence and not the flux dependence. One therefore should be cautious when interpreting the results of $\mathrm{H}_{2} \mathrm{O}$ and $\mathrm{CO}_{2}$ release by CACECO.

The runs that have been made have assumed a $50 \mathrm{~kW}$ heater in the sodium pool. The experiment will not use constant power in the heater, but will adjust it as a function of time to maintain constant pool temperature. This effect will need to be incorporated into CACECO by trial and error.

\subsection{Acoustic Wave Measurements in Concrete}

The comprehensive program to study CRBRP concrete during its initial 90 days of cure has been completed. This study used acoustic waves, weight measurements, and comprehensive strength tests to study the effect of the various ingredients on the curing process. In particular, five batches of concrete were tested. Batch 1 was simply a cement-water mix; batch 2 added flyash to batch 1; batch 3 added fine limestone aggregate to batch 2; batch 4 added coarse limestone aggregate to batch 3; and batch 5 added a water reducing agent and an air entraining agent to batch 4. Batch 5 is the proposed CRBRP concrete. For these tests, the acoustic wave measurements on each batch were used as an indicator of the cure and the weight measurements were used to monitor water retention. The effects of changing environment on the curing process were minimized by storing all specimens at room temperature and 100 percent relative humidity.

The results of this program describe several interesting features about the curing process of CRBRP concrete. First, the water retention characteristics of the 
concrete become more and more stabilized from batch I to batch 5 . Batch 5 stabilized after 10 days of cure with a net loss of 0.4 percent by weight of water. Second, the addition of the flyash and the aggregate had the most dramatic effect on the curing process -- the addition of the flyash slowed the curing process and changed its form while the addition of aggregate increased the strength of the concrete, but did not appreciably affect the curing process. Third, the addition of admixtures to the mix reduced its ultimate compressive strength, but did not affect its water retention characteristics. And, fourth, an empirical relation between acoustic wave velocity and compressive strength has been obtained; thus providing a nondestructive technique for monitoring the cure in this variety of concrete.

\subsection{Cell Liner Stress Analysis}

The cell liner analysis is concerned with calculating the possible growth of undetected flaws in an A515, Grade 55, steel liner during the first critical seconds of a major sodium spill. The analysis consists of a transient thermal solution to a postulated sodium spill followed by a thermal stress analysis of the liner. From the stress field and fracture data for the liner material some determination of possible crack growth can be made.

A finite element model for the liner analysis has been constructed. The analysis assumes an axisymmetric steel liner fixed at the edges with the sodium spill occurring at the centerline with a predetermined radius. The current configuration employs a liner thickness of $1 / 4$ inch with a plate diameter of 20 inches and a spill diameter of 8 inches. A parametric study will be made with the spill radius and plate thickness as variables. The effect of the film coefficient used in the transient thermal analysis will also be determined (currently a value of $6000 \mathrm{Btu} / \mathrm{hr} / \mathrm{ft}^{2} \mathrm{O}_{\mathrm{F}}$ is being used).

For the current geometry a thermal stress analysis has been completed to steady-state conditions. Large plastic stresses (greater than 1 percent) are observed in the liner directly beneath the spill and occur completed through the thickness. Based upon the radial stress distribution across the liner, the possibility of flaw growth in a liner weld region cannot be ruled out. 


\section{RESEARCH FOR ELEVATED TEMPERATURE DESIGN CRITERIA}

(R. E. Nickell, 5431; C. M. Stone, 5431; D. W. Lobitz, 5431;

C. H. Karnes, 5835; J. A. Van Den Avyle, 5835; 0. J. Burchett, 9352;

J. H. Gieske, 9352; W. B. Gauster, 5111)

\subsection{General}

Two reports were issued during the period:

(a) "Nondestructive Evaluation of Creep-Fatigue Damage," by R. E. Nickell (5431), SAND 77-0076, and

(b) "Bibligraphy of Nondestructive Testing Reports and Other References Pertinent to NRC Contract 189a, No. A1068,"

by D. W. Ballard (935I), SAND 76-0748.

Due to scheduling conflicts and the press of other commitments, Dr. S. W. Key (1281) will accompany Dr. Thomas J. Walker (NRC) to Japan for the 3rd International Conference on Pressure Vessel Technology and subsequent technical discussions with the Japanese.

\subsection{Subtask on Creep-Fatigue}

Fatigue and creep-fatigue tests, in air, for unwelded $2 \frac{1}{4} \mathrm{Cr}-1$ Mo steel specimens are complete. Five tests were carried out. Two of these were pure fatigue tests at \pm 0.25 percent strain and \pm 0.50 percent strain trat were cycled to failure. Three tests were combined creep-fatigue tests with hold times ir tensior, compression, or tension-compression. In order to approximately equelize the damage between creep and fatigue, relatively short holi times were used in the strain-controlled mode -- three minutes for tension-compressior and six minutes for either tension or compression. Beceuse of uncertinty as to the effect of oxidation in air on fatigue crack growth, all tive experiments are being repeatea in an argon atmosphere.

Some ten straight (es opposed to hourglass-ehaped) specimens of velded $2 \frac{1}{4} \mathrm{Cr}-1$ vo steel have been deliverea to "ar-mest for fatigue and crefp-fatigut testing. The weld/base-retal interface is in the center of the $\frac{1}{4}-i n c h$ diameter specirens, and the weld retal extends to include one of the trieaded ends. it the 
same time creep testing of welded $2 \frac{3}{4} \mathrm{Cr}-1$ Mo has been initiated at Sandia, using $\frac{2}{2}$-inch diameter creep specimens with the weld/base-metal interface also in the center. One creep test ( $14 \mathrm{Ksi}, 1100^{\circ} \mathrm{F}, 350$ hours) has been completed, carried out to the onset of tertiary creep. The 350-hour duration agrees with data for the unwelded $2 \frac{1}{4} \mathrm{Cr}-1 \mathrm{Mo}$, which is not surprising, since the necking and large straining ( $\sim 6 \%$ ) occurred on the base metal end near the specimen shoulder. Based on this one test, there is some question as to the need for continued creep testing of welded $2 \frac{1}{4} \mathrm{Cr}-1 \mathrm{Mo}$. No creep damage in the weld metal is likely.

Sixteen straight-sided fatigue and creep-fatigue specimens have been machined from the block of 316 stainless steel from ORNL. Ten of these will be shipped to Mar-Test for high temperature tests similar to tests conducted on $2 \frac{1}{4} \mathrm{Cr}-\mathrm{I} \mathrm{Mo}$, while the remaining six will be subjected to varying degrees of fatigue damage, at room temperature, at Sandia as a calibration exercise for the positron annihilation investigations. We are awaiting information from NRC on the creep testing to be carried out at Brookhaven National Laboratory on a similar block of 316 stainless steel. In anticipation of future tests on this material, a high-temperature (1100 K) aging process has begun on blocks of 316 .

To date two ultrasonic examinations of fractured fatigue samples of unwelded $2 \frac{1}{4} \mathrm{Cr}-1$ Mo have been conducted with negative results. Such findings are not surprising in fractured samples since pure fatigue damage occurs primarily at the fracture surface. The ultrasonic devices only see end effects at such free surfaces. A clearer picture will emerge from inspection of the damaged, but unfractured, specimens.

Computer and electronics orders for the multiaxial creep-fatigue facility have been processed, and MTS Systems Corporation is returning a quote for the test frame sometime during the month of March. Analysis is continuing in order to determine the test matrix on this facility. An elastic-plastic analysis has indicated that the scoping calculations carried out in the previous quarter were sufficiently accurate. An elastic-plastic-creep analysis is underway in order to determine the effect of creep deformation on the distribution of deviatoric and dilatational stresses in the tube wall. 


\subsection{Subtask on Cell Liners}

The nonlinear thermal analysis of an A515, Grade 55, steel liner, subjected to sudden thermal shock over a finite area due to an accidental sodium spill, has been completed for a particular set of parameters $\left(\frac{3}{4}\right.$-inch thick liner, 8-inch diameter spill area, heat transfer coefficient about $6000 \mathrm{Btu} / \mathrm{hr} / \mathrm{ft}^{2} \mathrm{O}_{\mathrm{F}}$ ); the subsequent elastic-plastic thermal stress analysis for this geometry has also been completed out to steady-state conditions. The plastic strains exceed 2 percent on the front surface and some plastic straining occurs completely through the thickness inside the spill area. From the radial stress distribution across the liner, the possibility of flaw growth in a liner weld region cannot yet be ruled out.

Further parametric studies remain to be carried out, using both larger and smaller heat transfer coefficients and larger and smaller spill areas. However, the need for some experimental support is becoming evident. Attempts to encourage continuous strain measurements on the HEDL LT-1 test were not successful; therefore, a controlled experiment at Sandia is in order, attempting to use capacitance displacement and strain gages that are modified slightly to eliminate problems with gas evolution from thermally-decomposing concrete.

In the meantime, a more realistic transient thermal analysis, including conduction and convection effects in the sodium, is being planned.

In terms of flaw characterizetion, there are four possible techniques to examine the welds of the cell liner. These are: (a) ultrasonics, (b) magnetic particle, (c) dye penetrant, and (d) eddy current. The magnetic particle, dye penetrant, and eddy current techniques are most sentitive to surface flaws. For a planar weld, these techniques are capable of finding flaws in the thousands of an inch range. Realistically, cracks running up to .020 inches should be found easily. The biplanar welds, however, present special problems for the magnetic particle and eddy current techniques. The dye penetrant technique should be as sensitive for the biplanar welds as for the planar welds.

For volumetric flaw detection, the only choice seems to be ultrasonic techniques. Here again the technique is most reliable for the planar welds. 
The planar weld can be examined with various angle beams from opposite directions as well as from straight-on. By calibrating with a test block according to ASME B\&VP Code specifications, one should reliably detect flaws or cracks as small as $3 / 32$ inch. For biplanar welds, the largest flaw which could escape detection may be twice as large since preferred orientation of the flaw may cause only a small portion of the signal to be returned in the direction of the ultrasonic probe. One is limited as to the angles one can use and a straight beam scan would be impossible. For this reason if flaws smaller than, say, .20 inches need to be found reliably in the biplanar welds, a new ultrasonic technique may need to be developed.

\subsection{Subtask on Creep Buckling}

Meshes for both the simplified elbow element and the doubly curved shell element have been created for the elbow geometry tested by the Japanese at the Power Reactor and Nuclear Fuel Development Corporation, O-arai Research Center, Ibaraki, Japan. The material is 304 stainless steel with a reasonably well understood creep law. Problems with the constraint routines in the MARC general purpose program have held up the analysis so far, but replacement routines have recently been obtained.

\subsection{Subtask on the Surveillance Test Loop}

Initial planning on the surveillance test loop is underway. A design task will be placed in the FY 78 budget, with construction to begin in late FY 78 and

FY 79. Cost figures and scoping calculations will be assembled in the current year.

\subsection{Other Items}

Mar-Test has submitted a proposal for FY 78 which calls for approximately $\$ 40 \mathrm{~K}$ of testing and $\$ 30 \mathrm{~K}$ of supporting microscopy. 25 The object is to extrapolate the effect of Iong hold times, in creep-fatigue tests, based on the changing microstructure of the material. The idea is similar to that proposed by Hale 26 for long-life creep failure prediction in $2 \frac{1}{4} \mathrm{Cr}-1 \mathrm{Mo}$. The idea seems to be of sufficient promise to warrant some investigation during FY 78 . 
(W. H. Buckalew, 5232; I. M. Choate, 5423; L. R. Edwards, 5423; J. A. Halbleib, 523]; D. A. McArthur, 5423; L. D. Posey, 5452; J. E. Powell, 5423; J. N. Sweet, 2151; S. A. Wright, 5423)

\subsection{Introduction}

The current status of research in the area of material motion detection diagnostics for reactor safety experiments is reported in this section. The purpose of this research is to study various material motion detection schemes, both experimentally and analytically, and to assess their potential as possibilities for existing and future safety test facilities.

In-core techniques that have application to medium- and large-bundle tests in facilities such as SLSF and SAREF are also being studied. They have the advantage of not requiring a slot in the test-reactor core but have inherently lower spatial resolution than the out-of-core systems for small-bundle and few-pin tests. Our studies have shown that the two systems may be competitive from the standpoint of resolution on large-bundle tests.

Several out-of-core, line-of-sight schemes are being investigated, including gamma-ray pseudoholography involving coded aperture imaging, electron beam flash $x$-radiography, and dynamic fission track devices.

\subsection{In-Core Fuel Motion Detection}

The main objective of the in-core fuel motion detection (FMD) program at Sandia is to test the feasibility of using large arrays of in-core detectors to monitor fuel motion in medium- to large-scale tests. This objective will be accomplished by mechanically simulating fuel motion in a 37-pin test assembly placed in SPR-III. The program is divided into three tasks: The SPR-III FMD experiment, detector research and development, and the unfolding analysis. Activities in each of these tasks during this reporting period were devoted to: 
a. Choosing the final design of the fuel positioning system.

b. Developing fabrication procedures for thin film thermocouples and diamond detectors.

c. Writing a computer code to evaluate the unfolding equation.

\subsubsection{SPR-III FMD Experiment}

The final design for the fuel positioning system was chosen. In this design the pins will be moved by pulling cables which run from the test assembly to the experiment station. The pins can be moved either individually or in groups. The pins are spring loaded into the reactor so that, when the experimenter relaxes tension on the cables, the pins will return to their initial unperturbed configuration. In addition to holding the pins in place with the cable system, a mechanical locking device is used to lock the pins in place. This device is under control of the reactor operator and will not allow the experimenter to move the pins (either in or out) without the operator's approval.

The final design drawings of the fuel positioning system and test assembly are about 70 percent complete. During the next quarter fabrication should begin.

During this past quarter the requirements of the fuel motion detector data acquisition system were defined. Briefly, the system must be able to sample up to 108 channels every $10 \mu \mathrm{s}$ and keep the noise and electronic pickup below $40 \mu \mathrm{V}$ RTI (referred to input). Because of the high speed and low noise requirements, the data will be amplified and digitalized near the signal source and stored in a buffer memory. From there the data will be sent to the Sandia computer system for unfolding.

In connection with defining the requirements for the data acquisition system, reactor experiments were also performed to test components of the system. The present experiments are being used to test grounding and noise reduction techniques. Later experiments will be used to determine the acceptability of preamplifiers, cables, connectors and other components of the system. 


\subsubsection{Detector Design and Development}

5.2.2.1 Fission Couple Detectors - During the past quarter, work continued on developing fabrication techniques for thin film chromel alumel thermocouples. The thin films are rf sputtered from targets constructed to our specifications by the MRC Corp. Auger composition analysis of the sputtered films indicates the atomic concentrations in the films are very close to those in the targets.

The Seebeck coefficients of the sputtered films were measured at room temperature with a two-point heated probe technique and the relative Seebeck coefficient, $\alpha_{\text {chromel }}{ }^{-\alpha}$ alumel , was determined to be $\approx 36.5 \mu \mathrm{V} /{ }^{\circ} \mathrm{C}$ as compared to $40.3 \mu \mathrm{V} /{ }^{\circ} \mathrm{C}$ from precision thermocouple tables. This agreement is felt to be quite good and indicative of the fact that high quality thermocouple films can be deposited.

A number of thermocouple test circuits have been fabricated on glass substrates, with the pattern definition performed by standard photolithographic processes. An example of such a circuit is shown in the accompanying photograph Figure 22. The thermocouple junction areas are $0.025 \mathrm{~mm}$ wide and the interconnecting thermocouple leads are $0.01 \mathrm{~mm}$ wide. Several circuits from a large array on a glass substrate are shown in Figure 23. The overall dimensions of the eight junction thermocouple networks are $0.10 \mathrm{~mm} \times 0.56 \mathrm{~mm}$. After $U$ and $W$ tabs are attached to the junctions, the circuits are separated by diamond sawing. Preliminary studies of the sawing process were initiated during this last quarter.

\subsubsection{Diamond Detectors - Work on diamond detectors progressed in three} areas: (a) the completion of new experimental facilities; (b) the fabrication of electrical contacts; and (c) the examination of detector properties. In the preceding quarterly, ${ }^{2}$ the initial attempts and the problems associated with obtaining good electrical contacts on diamond were described. Also, in this quarterly, the discussion with S. F. Kozlov (the inventor of the diamond detector) on the contact problems was summarized. The current approach to resolving the contact problems follow some of Kozlov's suggestions. 


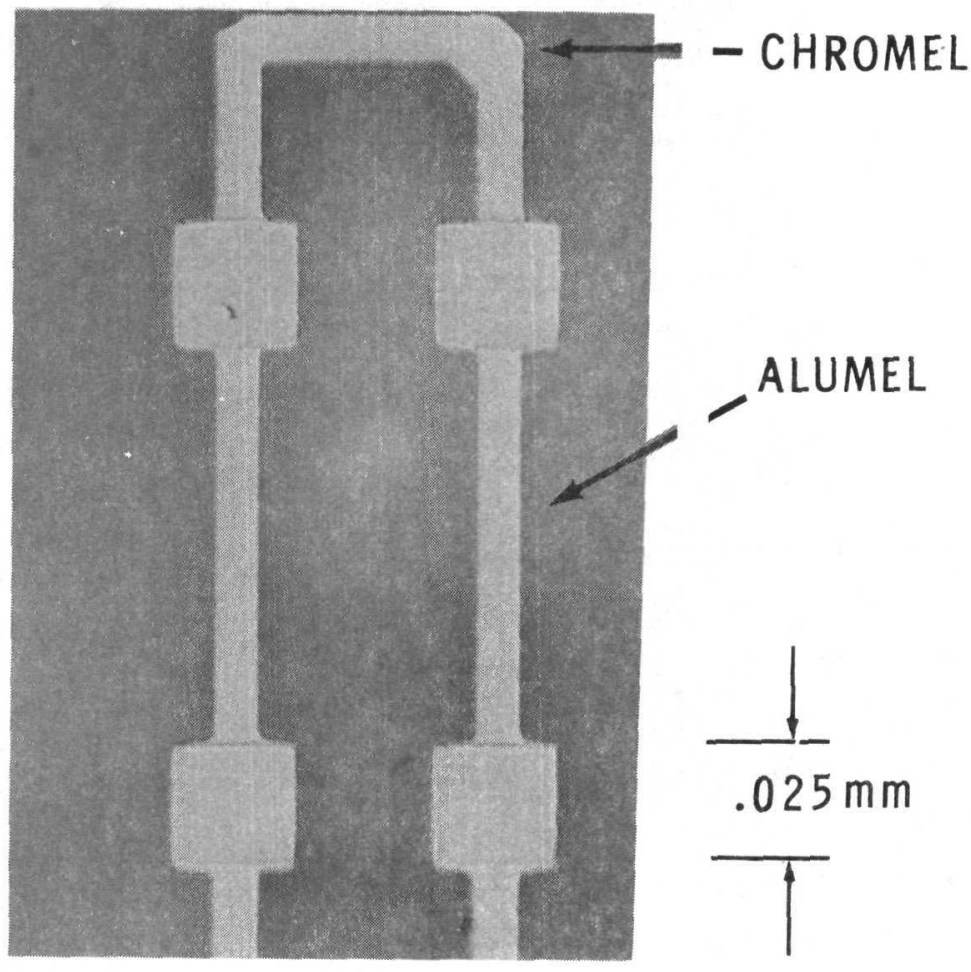

Figure 22. Thin Film Thermocouple unction

(The chromel and alumel thin

film overlap at the junction) 


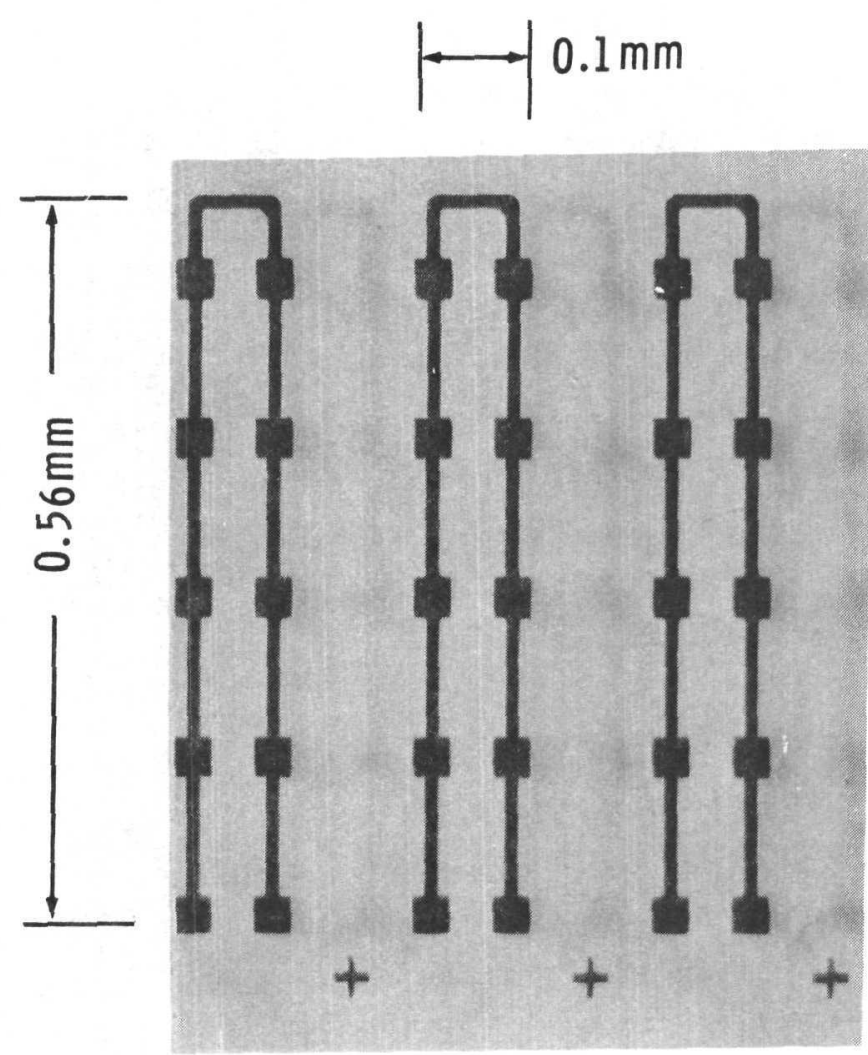

Figure 23. Eight Junction Thermocouple 
A significant fraction of the past quarter was expended on completing the high-temperatures, high-vacuum graphite furnace facility and the nuclear spectroscopy system. Both systems are now functional. Several diamond detectors were fabricated with silver paint injecting contacts using the new furnace system. For those detectors which were heat treated at $600^{\circ} \mathrm{C}$, the contact bond was mechanically very rugged. These detectors counted $\mathrm{Am}^{241}$ alpha radiation with no evidence of polarization, but the pulse shape was disappointing. In real time the pulses were very broad. The I-V (current-voltage) measurement of the injecting contact did not exhibit the expected trap-filled limit behavior. This indicates that the silver may not have diffused far enough into the diamond lattice to give appropriate band bending for good injection; however, this is in conflict with the fact that there was no space charge polarization effect. The poor pulse shape could be explained if the Schuebweg (the average distance an electron or hole travels before trapping) is less than the thickness of the detector, but this does not explain the I-V characterization.

Work is now in progress to resolve these problems. Different sample thicknesses, different contact materials, and alternate heat treatment schedules are planned. From the work on contacts thus far, it has become apparent that the diamond surface contaminates easily at elevated temperatures. Because of this surface contamination, it has become of concern that the optical absorptionannealing experiments reported earlier ${ }^{8}$ may be in error, especially at temperatures above $500^{\circ} \mathrm{C}$. An experiment was performed to determine the effect of surface contamination on the optical absorption. The results showed that the optical transmission is quite sensitive to the surface condition. This observation indicates that the earlier data are probably better than what was reported. 8 Some of the optical absorption-annealing experiments will be repeated concommitantly with the detector radiation experiments.

\subsubsection{Unfolding and Transport Calculations}

An Integral equation was described in the previous quarterly report which relates detector response to changes in the fuel density. 9 
For purposes of unfolding, this equation can be expressed as

$$
\Delta R_{i}=\int k_{i}(\underline{r}) \delta \rho(\underline{r}) d \underline{r}
$$

where

$$
\begin{aligned}
\Delta \mathrm{R}_{i} & =\text { change in the } i \text { th detector } \\
k_{i}(\underline{r}) & =i \text { th detector response kernel } \\
\delta \rho(\underline{r}) & =\text { fractional change in density at } \underline{r} \\
\underline{r} & =\text { position vector. }
\end{aligned}
$$

This equation is a Fredholm integral equation of the first kind and can be solved for $\delta \rho(\underline{r})$ given the detector responses and the kernels using the Sandia unfolding code IUNFOLD.

A computer code called RHOPER (for $\rho$ perturbation) was written to calculate the detector response kernels. The kernels are obtained by integrating forward and adjoint fluxes weighted by the cross sections over energy and angle (see Reference 9) The code performs the angle integrations by using the spherical harmonic moments of the angular fluxes. This method of angle integration has the advantage of reducing the size of the forward and adjoint flux arrays compared to angle integration using the $s_{n}$ quadrature angular fluxes. In addition, it is easier and quicker to calculate the flux moments when using Monte Carlo transport calculations than the angular fluxes for numerous discrete directions. The present version of the code uses only the zero ${ }^{\text {th }}$ order moments (i.e., scalar fluxes) to perform the angle integration. Later versions of the code will include the higher order flux moments.

In order to determine the validity of the unfolding equation (Equation I) and our method of evaluating it, the code will also integrate the product of the detector response kernel times the change in fuel density to determine a detector response for a given change in fuel density. The results of these calculations are then compared with normal method of determining dose to a detector 


$$
\Delta R=\int\left(\phi_{p}-\phi_{0}\right) \Sigma_{d}(\underline{r}, E) d \underline{r} d E
$$

where

$$
\begin{aligned}
& \Delta R=\text { change in detector response } \\
& \phi_{p}=\text { perturbed flux } \\
& \phi_{0}=\text { unperturbed flux } \\
& \Sigma_{d}=\text { detector cross section at } \underline{r} .
\end{aligned}
$$

The fluxes $\phi_{o}$ and $\phi_{p}$ are obtained by performing two forward transport calculations, one for the unperturbed geometry and another for the perturbed geometry. The present version of RHOPER underestimates the detector response by a factor of two. We believe this difference will be removed when the higher order flux moments are taken into account.

\subsection{Electron Beam Flash X-Radiography}

(W. H. Buckalew, 5232; L. M. Choate, 5423; L. D. Posey, 5452)

During this quarter a new radiography system for use in the coded source flash $x$-ray cinematography program was designed. It is hoped that the present system design will provide (a) a more intense and more uniform electron beam at the convertor plane, (b) a more controllable electron beam, (c) an increase in modulation from the coded convertor, (d) a substantial reauction in background $x$-ray contributions, and (e) better collimation of the desired coded x-ray beam. A conceptual schematic representation of the system is shown in Figure 24 (side view) and Figure 25 (top view). The system corsists of the following basic components: vacuum chamber, linear cathode, linear coded convertor, focusing and sweeping magnets, and shielding and collimating system. Each of these components will now be discussed in some detail.

The radiography system is designed for operation on HERMES II ( $13 \mathrm{MeV}$ end point electron energy, $125 \mathrm{kA}, 70 \mathrm{~ns}$ ). The standard hermispherical cathode of HERMES II produces an annular beam which is about 8 inches in diamet If the 


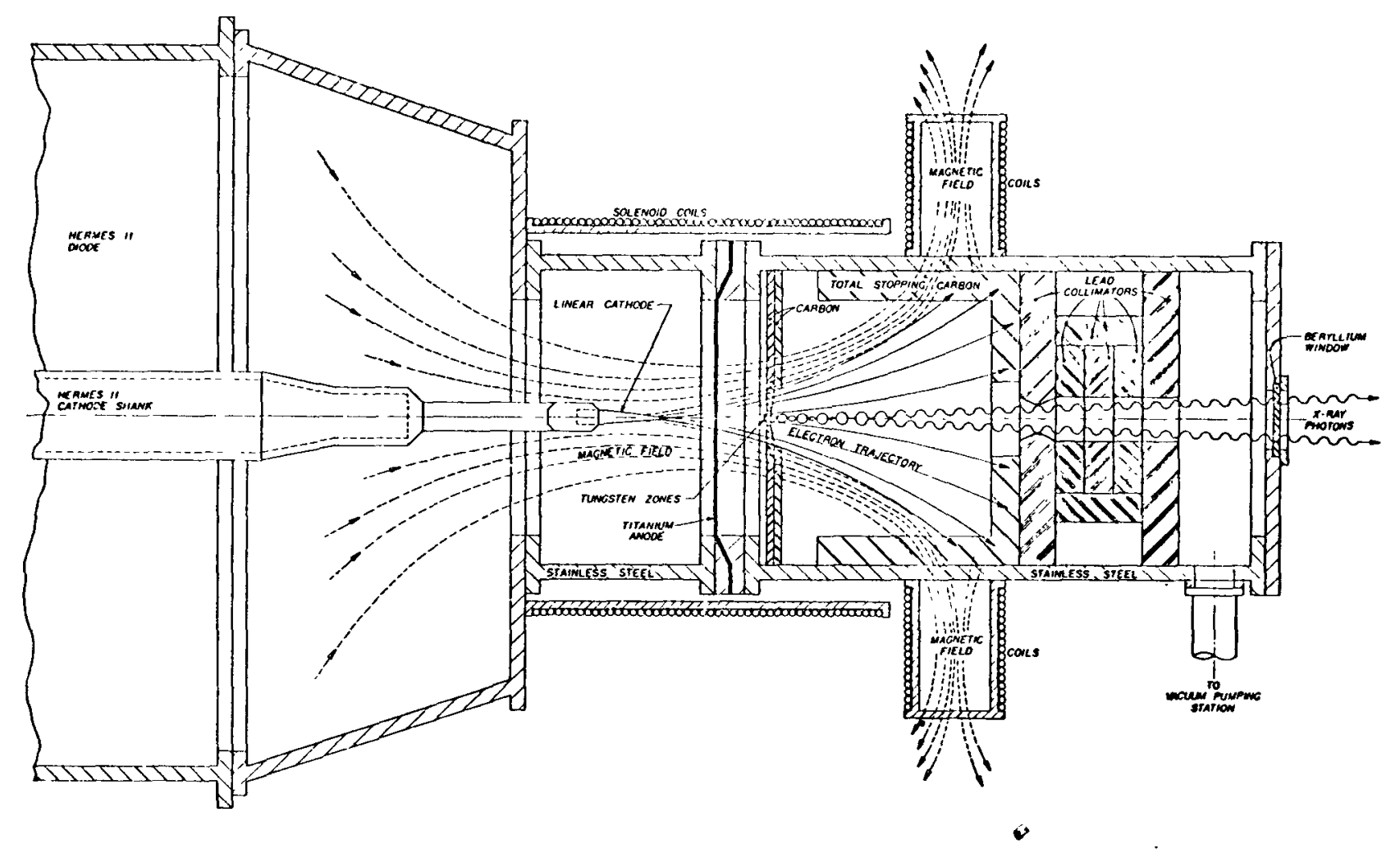

Figure 24. Hermes II Coded Source Flash X-Ray Chamber Proposal (side view) 


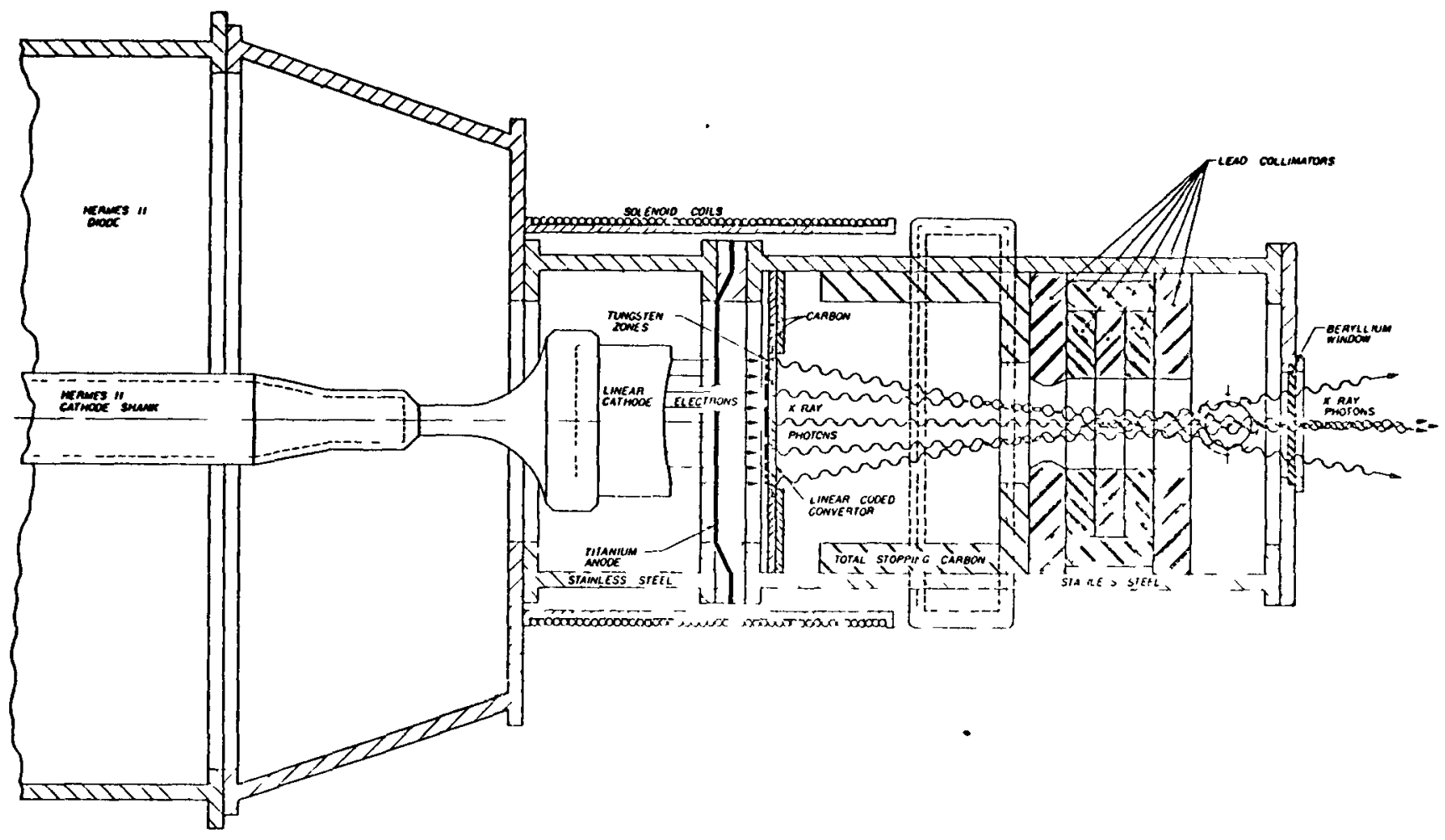

Figure 25. Hermes II Coded Source Flash

X-Ray Chamber Proposal (top view) 
intensity at the center of the beam is normalized to 1.0 , then the intersity of the beam at a position 3.5 inches from the center is approximately 1.5 . Therefore, the electron beam hitting the linear coded convertor ( $\frac{1}{<} \mathrm{x} \&$ inches effective area) just in front of the titanium anode is nonuniform. Furthermore, nost cf the electron beam does not hit the convertor and these unwanted electrons ultimately produce unwanted background bremsstrahlung when they collide with the chamber walls or collimators. Consequently, a linear cathode has been designed for operation on HERMES II. This cathode should provide a more adequate electron beam (more uriform and more intense) of about $2 \times 10$ inches. Preliminary experimental investigation of several linear cathode designs indicates that an acceptable configuraticn can be found.

A redesign of the linear coded convertor has been completed in order to substantially reduce background bremsstrahlung contributions from the substrate. Calculations performed to estimate the amount of modulation attainable with various convertor configurations indicate a forward 10 degree bremsstrahlung energj flux modulation of about $9: 1$ for 60-mil W mounted to a 5-mil Ti substrate. Structural integrity of this convertor design has yet to be shown. Several bonding techniques are presently being investigated and include bonding by several types of eroxy resins, bonding by electro-welding, and mechanical bonding. The final convertor configuration will consist of about 25 w zones mourted to a 5-mil Ii substrate such that integrity will be maintained at energy depositions of about $100 \mathrm{cal} / \mathrm{cm}^{2}$ pulse.

The effective elimination of unwanted electrons fassine throukh and anourd the convertor has been a problem in the past. As stater earlier, thesf eleatrons produce unwanted background bremsstrahlurg when coliicing wits tre corvertor. substrate, chamber walls, and collimators. In order for the electron beam tc "blow up" after passing through the anode, the bear must be injected into a vacur region with a pressure of Iess than $20^{-3}$ rorr. The present chamber desigr croula provide for a vacuum of about $10^{-5}$ Torr.

To further aid in the "sweep out" of there urwarted flcctrons, exteral magnetic fields have been added to the chamer to "sweoc" the ejectron into a

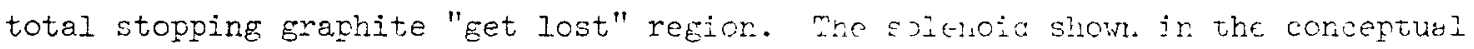
representation of the system is desigrea ir modules to allow for come flexibility in operation. The ragretic field of the solenoid will also aid in fucusing the 
electron beam from the linear cathode to the convertor. The magnetic field lines of the solenoid are "clamped" to the chamber walls by the field lines of the two "bucking" magnets. The strength of the magnetic fields should be about 10 kilogauss.

The shielding and collimating system is designed so that background bremsstrahlung will be preferentially shielded from the exit aperture. The coded X-ray beam will pass through a beryllium window and should provide a field of view of about 3 inches radially and 12 inches axially at source-to-object/object-to-image distances of $2.0 \mathrm{~m} / 2.0 \mathrm{~m}$. This field of view can easily be increased once system performance has been evaluated.

A quantitative evaluation of the effects of photon buildup upon spatial and areal density (mass) resolution capabilities in poor geometry situations began this quarter. Experiments were initiated this quarter on a LINATRON $1500(5.0 \mathrm{MeV}$, $7.5 \mathrm{MeV}, 10.0 \mathrm{MeV}$ with a focal spot $\sim 1 \mathrm{~mm}$ ) to resolve some of the questions. Although the data are still under analysis and some experiments are still being performed, preliminary results indicate qualitatively that photon buildup is more of a problem in mass resolution degradation at lower energies, and that careful filtering can substantially reduce these effects.

\subsection{Coded Aperture Imaging}

\subsubsection{Joint NRC/ERDA Assessment of SAREF Diagnostics Reguirements and Capabilities}

In November 1976 , a Steering Group was formed of representatives from ANL, Sandia, IASL, ERDA and NRC to assess the various candidate diagnostic methods proposed for SAREF, with particular emphasis on STF diagnostics. Sandia and IASI were asked to review the SAREF diagnostic requirements provided by ANL, and recommend changes to them if necessary. Each major diagnostic technique proposed for STF (hodoscope, coded aperture, flash X-radiography, in-core detection, and in-situ radiography) is represented by a working group composed of representatives from ANL, Sandia and LASL. After a concensus has been established on the diagnostic requirements, the working groups are to compare the existing diagnostic capabilities with the requirements to decide what diagnostic equipment needs to be developed and to recommend a program of research to develop the needed equipment. 
At Sandia, a group of ten analysts and experimentalists reviewed the ANL diagnostic requirements in several meetings, and concluded that some modification of the ANI requirements would be necessary. Similar conclusions were reached by IASL. A meeting of personnel from LASL and Sandia was held in February to define an alternate set of diagnostic requirements. In this meeting, tentative requirements were set independently of any particular safety issues. The required accuracy in the fuel density measurement was stated precisely, and the effects of material motions on the diagnostic requirements were also considered. Additions and small changes are still being made to this tentative set of requirements and the final set of alternate requirements will be reported in the next quarterly report. 
$\bullet$

0 


\title{
6. ACPR FUEL MOTION DETECTION SYSTEM
}

\author{
(J. R. Kelly, 5423; K. T. Stalker, 254I; S. R. Dolce, 1III)
}

\section{I Introduction}

During December 1976 one of the major milestones in the program to develop the fuel motion detection system was reached. An LMFBR fuel pin placed next to the Sandia Pulsed Reactor (SPR-II) was imaged both with X-ray film and with an active recording system. First, in this section, the SPR II experiment will be explained in detail. Second, the analogue and digital reconstructions will be discussed. Third, the tests of scintillator resolution and possible methods to improve their performance will be presented. Fourth, the progress recently achieved in preparation of the Collimation Test Module (CTM) for the final major feasibility test to find out if adequate signal to background can be obtained at ACPR will be discussed. Finally, signal-to-background estimates will be presented.

\subsection{SPR II Fuel Pin Imaging Tests}

A very important question, which must be satisfactorily answered if a viable fuel motion detection system is to be developed, is the following. Can a fuel pin excited by neutrons from a nearby reactor be imaged with high resolution and with good signal to noise and background ratios with an active recording system? The SPR-II is an extremely useful source for examining this question. Because it is a fast reactor and not surrounded with moderator, the assembly of experiments in its vicinity is greatly simplified. Furthermore, for this fuel pin imaging experiment, the reactor was operated for the first time next to the building shield wall beside the line-of-sight of the beam port. In this configuration, the kiva wall shielded the detectors and the electronic recording instruments. Finally, our estimates indicated that the peak gamma-ray flux at the detector from the pin would be very close to that from a pin exposed to a $500 \mathrm{cal} / \mathrm{g}$ irradiation at ACPR.

The experimental configuration is shown in Figure 26 . The reactor was placed $60 \mathrm{~cm}$ from the building wall and $25 \mathrm{~cm}$ from the fuel pin collimator line-of-sight. Polyethylene blocks $5 \mathrm{~cm}$ thick were placed on each side of the pin to boost the thermal neutron flux and to make the spectrum similar to that of the ACPR. The collimator in the beam port was made of disks of $\mathrm{B}_{4} \mathrm{C}$-loaded paraffin and disks of 


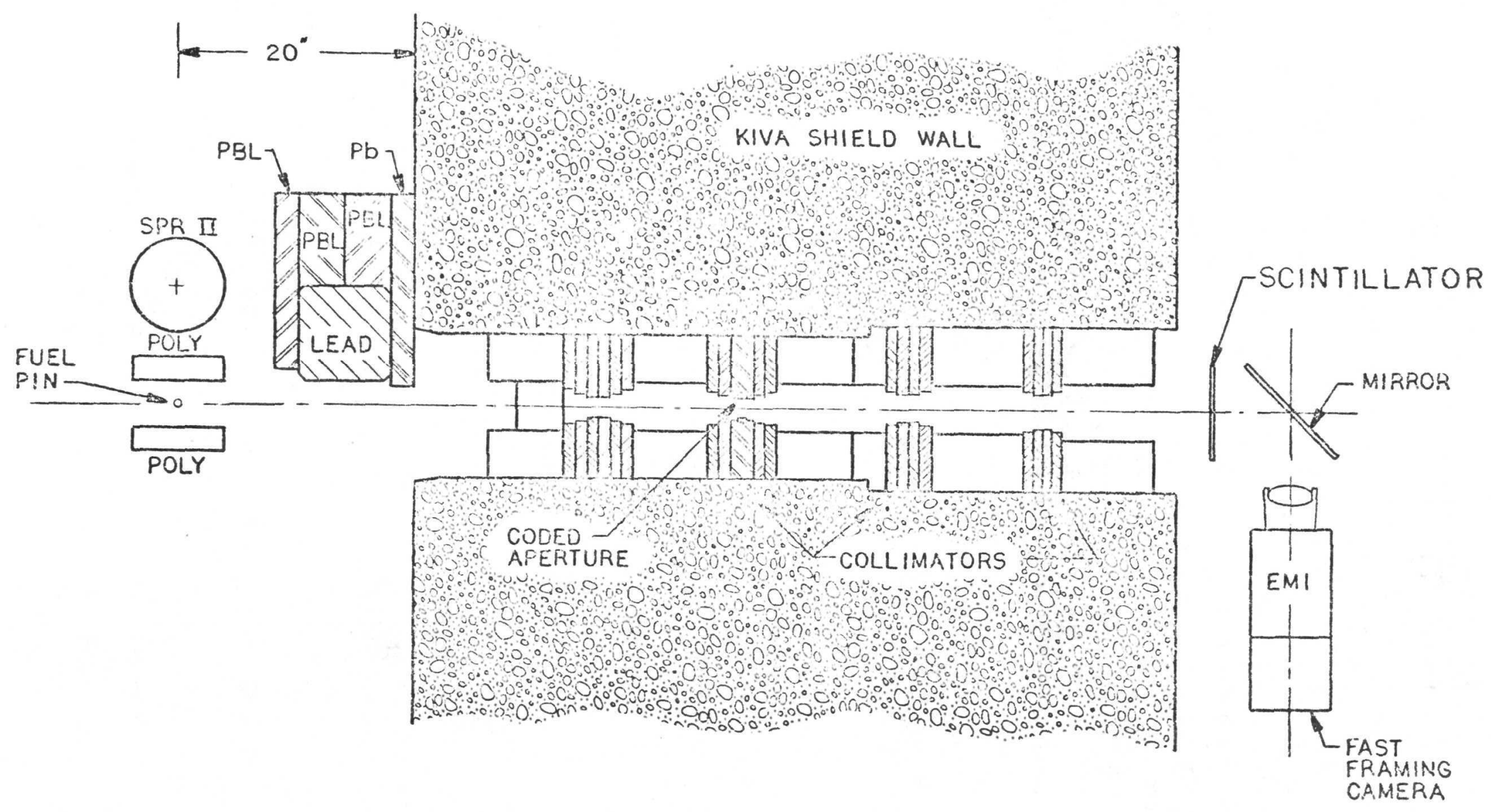

Figure 26. Configuration for the Fuel Pin Imaging Experiment at SPR II 
lead. After the first experiments, the collimator materials beyond the third lead collimator were removed because they were found to be unnecessary. Near the detector location (labeled scintillator), X-ray film, scintillators, or the X-ray image intensifiers were placed. When a scintillator was used, the mirror diverted the optical shadowgram so that the optical image intensifier would not have to be in the direct $\gamma$-ray beam.

Dosimetry measurements nearby and in line with the collimator revealed the following radiation fields. For a $300^{\circ} \mathrm{C}$ burst (the fuel temperature in the reactor rises to $300^{\circ} \mathrm{C}$ ), the dose behind the wall was 9 mrads. Iust to the side of the beam, when the collimator was open, the dose was 19 mrads, and in the beam without the fuel pin in place it was 35 mrads. This was an encouraging result because the dose from the fuel pin was expected to be 30 mrads. Subsequent analysis of the dose measurements have indicated that most of the background came from two sources. Part came from scattered $\gamma$-rays in the vicinity of the coded aperture, and most of the rest came from portions of the polyethylene blocks which were in the field of view in the early experiments.

The first imaging tests were made with a $93 \%$ enriched pin and were recorded on Kodak type AA lead pack X-ray film. The exposures were, of course, very low because the film is relatively insensitive. This is much of the reason the fuel motion system requires the use of image intensifiers. Nevertheless, exposures made with a sum of 3 reactor bursts showed a faint structure that clearly exhibited the proper pseudohologram characteristics for a fuel pin. The pin structure disappeared when the pin was removed. The early exposures also showed that reactor $\gamma$-rays scattering in the polyethylene blocks and lucite pin holders were imaged and contributed background. These scatterers were removed from the field of view in later exposures.

Although the film exposures were very low, images of the fuel pin were obtained and they will be discussed in the section on reconstruction.

A number of combinations of active recoräing were tested. Each configuration is discussed below.

(a) Scintillator, Optical Image Intensifier, Open Shutter Camera

Although the preliminary tests of scintillator spatial resolution conducted at the ${ }^{252} \mathrm{Cf}$ facility revealed poor modulation and resolution 
for all of the .05-, .1- and .2-cm thick NaI crystals, they were nevertheless tested here with the optical image intensifier. It was found that the system easily had enough gain to record signal intensities 10 times lower than expected in the peak of an ACPR burst, and that the pin pseudohologram structure could be seen on the film. However, that structure is poorly defined and no image of a fuel pin has been obtained from these data as yet. The scintillator resolution is the problem and it will be discussed more thoroughly in the section on scintillators.

(b) Scintillator, Optical Image Intensifier, Framing Camera

The pseudoholograms were also recorded at l-ms frame periods and again reveal adequate gain in the system. The SPR-II reactor pulse width with the polyethylene in place is only $200 \mu$ s so there is no way to properly display any fuel motion in the burst mode. Even with very fast system response, only one frame on the film would be exposed. (In fact, about 5 frames were exposed because of the few millisecond response of the optical image intensifier. As has been mentioned in earlier reports, the manufacturer is about to market a faster response tube.)

(c) X-ray Image Intensifier, Open Shutter Camera

The shadowgrams recorded with this combination showed satisfactory exposure, contrast, and spatial resolution. The fuel pin structure was evident, and images of the pin were reconstructed directly from the camera fi.m without the use of enhancement techniques. Those images will also be discussed in the reconstruction section.

\section{(d) X-Ray Image Intensifier, Framing Camera}

This combination revealed a difficulty with the framing camera. Once again the principal film exposures were spread over about five l-ms frames. These exposures, however, did not appear to be in good focus. At 
the same time, there was not evidence of blurring due to a relative motion of image and film during each frame. However, it happened that when the camera was stopped after the burst, the shutter was open and as a consequence the weak delayed phosphor image of the intensifier was integrated for about 5 minutes. This shadowgram is very sharp. The conclusion has been that the focus or film position shifts when the film moves. This problem is being investigated but it is not considered serious.

Although the reconstructable pseudoholograms at this stage have only been obtained with open shutter exposures, we feel this has little influence on the conclusion that fuel pin images can be recorded in ms-frame times, with good exposure and adequate spatial resolution with an active recording system. A new pin register camera is being purchased which has $f / 1.9$ optics (the same $f$ number as used in the open shutter exposures). The film in this camera is at rest when the shutter is open. It is at least 20 times more sensitive than the rotating prism camera used here and has a frame rate of $10^{3} /$ second.

The important conclusions that can be drawn from the SPR-II tests are the following:

(a) Signal levels from fuel pins in the vicinity of the reactor can be reliably predicted. (In fact, only once during the entire series were the signal, gain settings, lens openings, etc., predicted for the burst found to be incorrect for proper film exposures.)

(b) A shield and wide field of view collimator structure can be fabricated which allows one to image a fuel pin illuminated by a reactor in close proximity to the pin.

(c) A scintillator exists which does allow high resolution, fast time response recording of fuel pin shadowgrams. The scintillator inside the $X$-ray image intensifier is composed of . I $\mathrm{mm}$ vapor deposited $\mathrm{CsI}(\mathrm{Na})$, and it recorded an excelient pseudohologram. 
(d) The gain of either active recording system is adequate to record pin pseudoholograms at l-ms frame periods during the burst. We also feel there is a good chance that recording can be extended for

I second at ACPR if the optical image intensifier is coupled with the pin register framing camera.

The above conclusions can only be made if similar signal-to-background ratios (estimated here to be $2 / 1$ ) can be obtained at ACPR. This is the reason for conducting the collimation test module experiments to measure signal to background at ACPR.

\subsubsection{Fuel Pin Image Reconstructions}

Images of the fuel pin have been made with laser and with digital reconstruction.

(a) Laser Reconstructions of the Film Radiographs

The film exposures were so weak that two stages of contrast enhancement in the radiographs had to be employed. This is a valid procedure provided that, at each stage, the density of the radiograph stays within its linear range. A l-to-l exposure was first made onto a high contrast mylar film (Kodaline 4567). This film has a fairly grainy structure when partially exposed but is fairly fast (as is needed for 1-to-l imaging in the fl6 photoreduction system which was available). This film was then photoreduced 10 to 1 onto a Kodak high precision glass plate. When placed in the laser reconstruction system with a $.0025-\mathrm{cm}$ wire de stop at the de focus, the reconstruction shown in Figure 27 was obtained. This is a composite of 3 pictures taken from the same photoreduction. The composite was necessary in this case because the laser beam was not coherent along the full length of the pseudohologram plate at one time. At the bottom is a dark horizontal band which is from scattered reactor $\gamma$-rays in a phenolic block which held the fuel pins. There are two darker bands down the center. These bands are the images of two fuel pins. They are negative on a lighter 


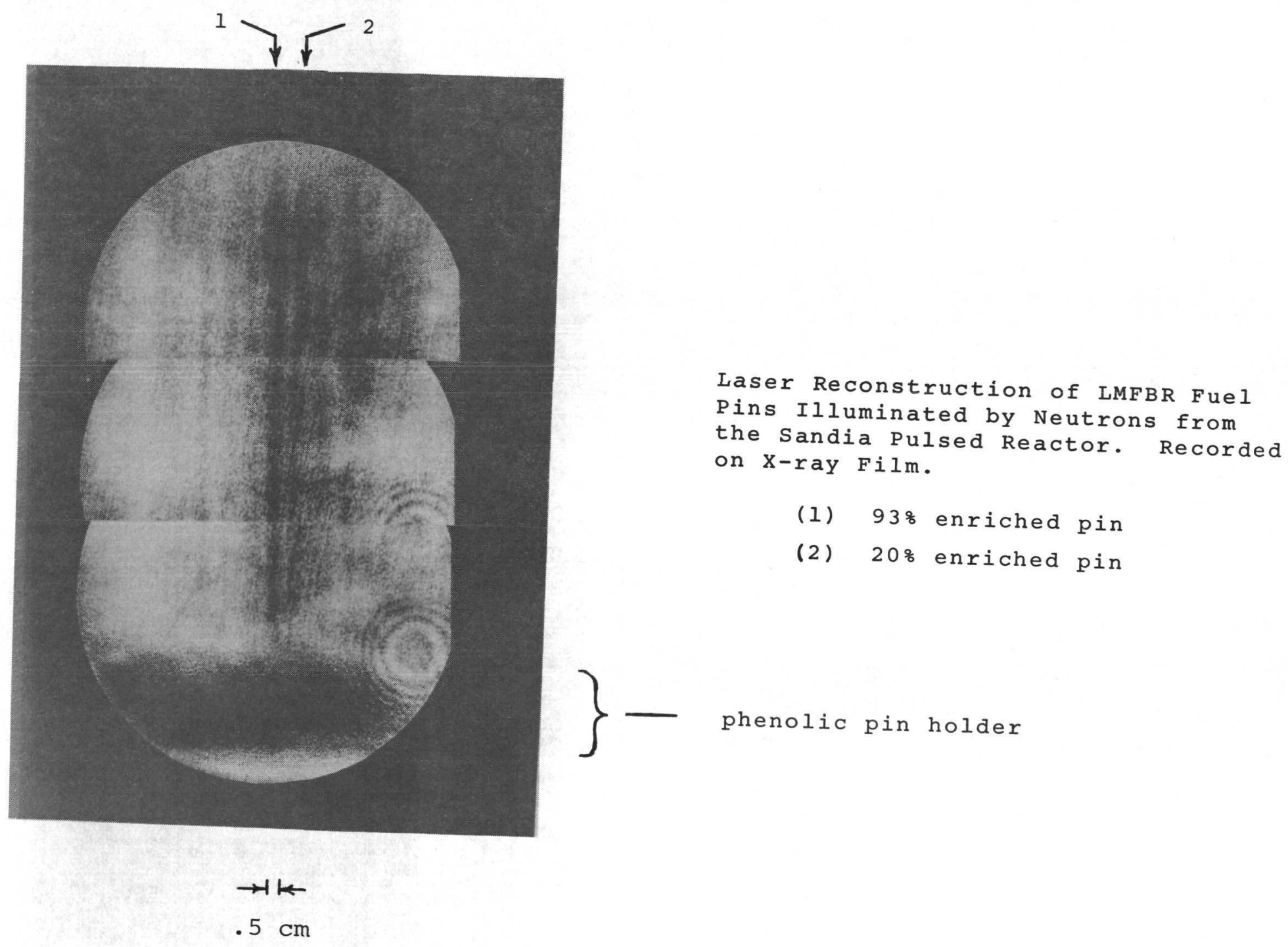

Figure 27. Laser Reconstruction of the Fuel Pin Recorded on X-Ray Film 
background because this exposure is negative. These two fuel pins are different however. The one on the left is $93 \%$ enriched in $235_{U}$ and the one on the right is only $20 \%$ enriched. Thus, the image above background on the left should be 4.6 times higher than the one on the right. The left pin is clearly darker, but the analysis of their relative intensities in this reconstruction has not yet been completed. The pin images show the correct structure for their having a diameter of $.5 \mathrm{~cm}$ and for observation with $2 \mathrm{~mm}$ resolution. One must keep in mind that no sharp edges will be seen even with a much higher resolution because the source intensity fades at the edges of a cylinder.

There are artifacts in the picture most of which can be identified. The circles on the left result from diffraction around a bubble in the reconstruction lens. Beside the pins are other faint lines which are the undiffracted light shadows of the coded aperture zones. (Usually if higher contrast is initially obtained in the radiograph, these other lines are so much weaker that they can barely be seen.) Also, only images of the pins go in and out of focus as a viewing screen is moved through the image plane. At the top, a few of the outer zone shadows are cut off by the final collimater and, consequently, the pin images are broader. Although this reconstruction does not show as clean a structure as was obtained with the ${ }^{252} \mathrm{Cf}$ simulations, it exhibits a very important feature of the pseudohologram recording process. That is, even if the recorded radiograph is improperly exposed, careful processing and analysis will still yield useful and quantitative results. This means that the system dynamic intensity range is much larger than was orginally expected.

(b) Laser Reconstructions of the X-ray Image Intensifier Open Shutter Camera Recorded Radiograph

$X-r a y$ intensifier recorded radiograph reconstructions are shown in Figure 28. In these cases, the contrast was good on the original film 
b 1
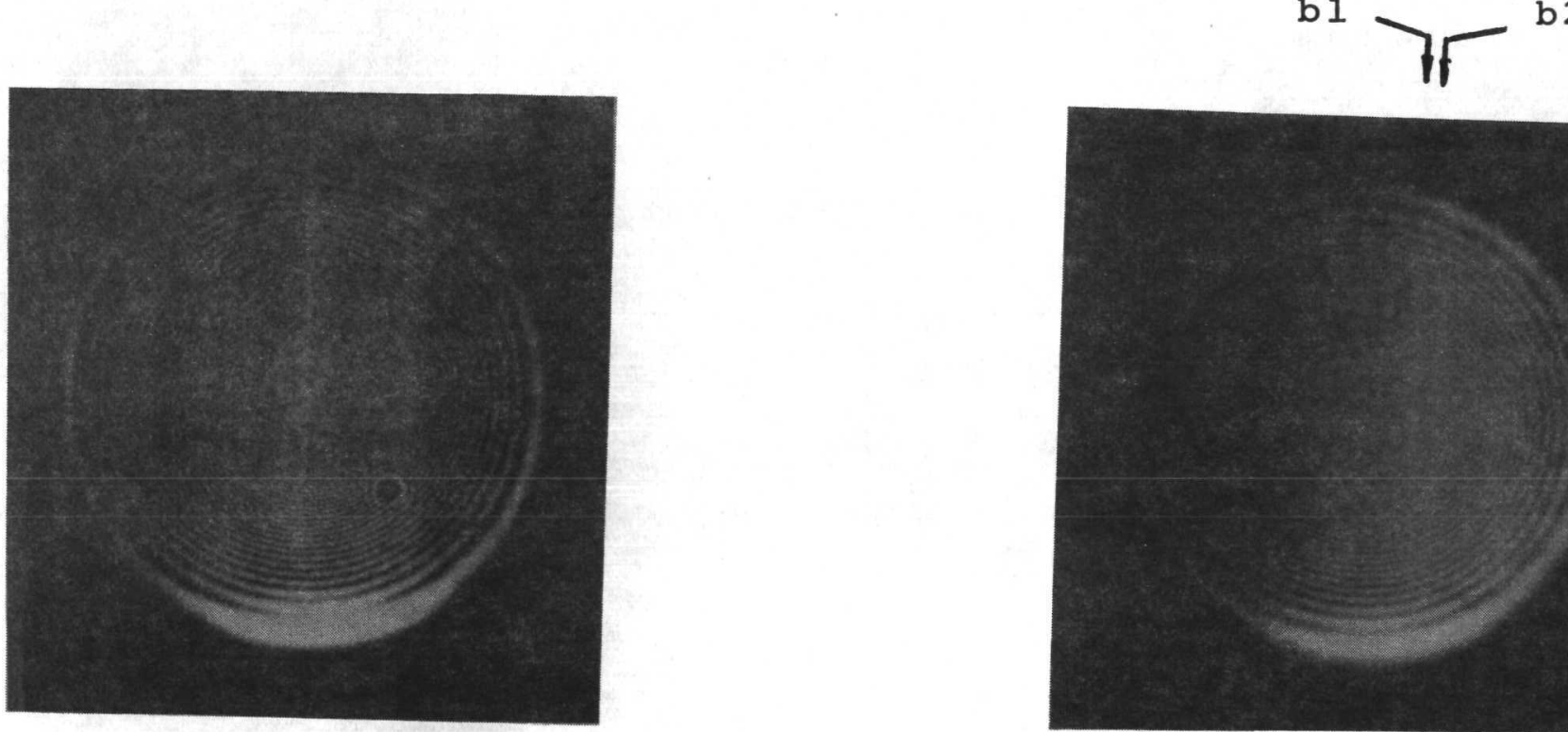

(a) Single Pin

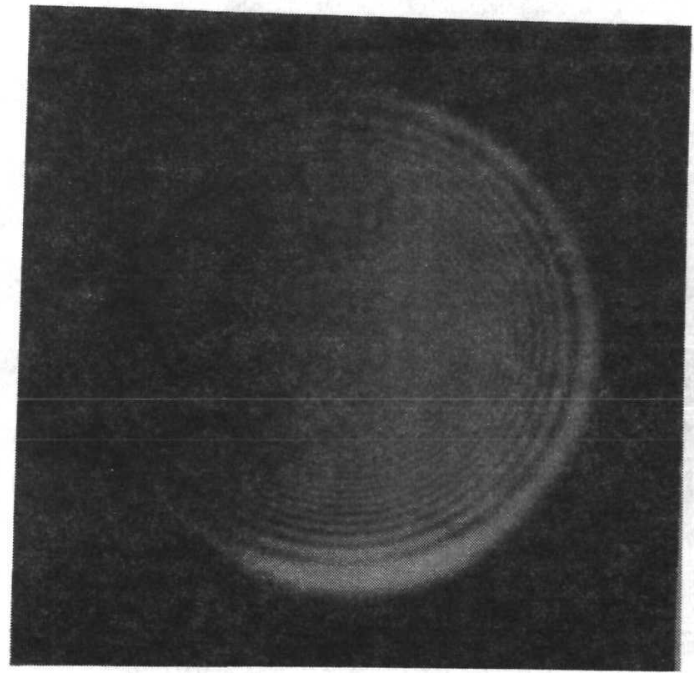

(b) Two Pins
(b1)
$93 \%$ enriched
(b2) $20 \%$ enriched

\begin{abstract}
Laser Reconstructions of LMFBR Fuel
Pins Illuminated by Neutrons from

the Sandia Pulsed Reactor. Recorded

with an $\mathrm{x}$-ray Image Intensifier.
\end{abstract}

Figure 28. Laser Reconstruction of the Fuel Pins Recorded with the X-Ray Image Intensifier 
and no enhancement or photoreduction steps were required. Also, no dc stop was used here. There is a series of rings superimposed on the image which are generated by the optical aperture in the system. These rings can be reduced by apodization and do not appear in digital reconstructions. The brighter vertical lines are the images of the pins. A single 93\% enriched pin is in Figure 28a and in Figure $28 \mathrm{~b}$ is a $93 \%$ enriched pin on the left and a $20 \%$ enriched on the right. In this case, the relative brightnesses of the two images are not as different as they should be because there was some exposure saturation in the recording system.

\section{(c) Digital Reconstructions of the X-ray Intensifier - Camera Recorded Radiographs}

Along with the analogue optical reconstructions already shown, a second reconstruction method employing the digital computer may also be used. Although the method is similar to that already described, ${ }^{27}$ some modifications must be made due to the use of a one-dimensional, instead of a circular, zone plate. Also because these pseudoholograms were scanned in a $256 \times 512$ or $512 \times 512$ resolution format to preserve the information present, extensive use of extended core storage (ECS) on the CDC 6600 computer was required. The general steps in the computer reconstruction will now be outlined. First, the pseudohologram is read by a scanning microdensitometer and the data are written on tape one row per physical record. When the tape is read, the density values are changed to transmittance values by dividing by the film gamma ( $\Delta$ Density/ $\Delta$ Log Exposure) and exponentiating. Since the data array is too large for storage in central memory, it is written one row at a time into ECS. The next step is to read the array from ECS a row at a time, subtract the average value of the array from each element and multiply by the complex phase factor $\exp \left[\left\{i \pi[I-(N / 2)]^{2}\right\} /\left\{R_{1}\right\}^{2}\right]$, where $i=$ $\sqrt{-1}$, I is the array element currently being considered, $N$ the number of array elements in a row, and $R_{1}$ is the radius of the central zone of the coded aperture in resolution elements. Next, the row is Fast Fourier Transformed (FFT). The multiplication by the complex phase 
factor and Fourier transformation is equivalent to Fresnel diffraction in the analogue optical system. To obtain the intensity, each member of the row is next squared in the complex sense and this intensity is written into ECS. Finally, after all rows are reconstructed and stored in ECS on a row by row basis, several rows (usually 64) are read into a subarray which is then plotted. There are several $(512 / 64=8)$ subarrays plotted to make the final output images shown in Figures 29 and 30.

Figure 29 is a computer reconstruction of the same single fuel pin previously shown in Figure 28a. The actual fuel pin is the long thin object in the center of the frame, while the dark band along the right is most probably an artifact caused by the chosen format used to scan the original pseudohologram. There is, however, some reason to believe that this dark band may also be the polyethylene moderator used in the experiment and viewed by only the outermost zones of the coded aperture. Further investigation to determine the origin of these bands is underway. Even though these unwanted bands are present, the long thin object in the center of the field is clearly the fuel pin since it does go in and out of focus as the focal parameter in the reconstruction algorithm is changed while the band structure at the edges remains unchanged. The scale factor in the vertical direction is different than in the horizontal direction because of the cylindrical phase factor used in the reconstruction process. In the direction along the pin (vertical), the scale in the reconstruction is the same as in the pseudohologram and represents about $135 \mathrm{~mm}$ length of fuel pin. In the radial (horizontal) direction, the scale magnification introduced by the one-dimensional reconstruction process is given by $N /\left(R_{1}\right)^{2}$ where $R_{1}$ is the radius of the central zone of the aperture expressed in resolution elements and $N$ is the number of resolution elements per horizontal scan line. In our case $\left(R_{1}\right)^{2}=500$ and $N=256$ so the horizontal magnification is .51 . on this scale the fuel pin image should be and is about eight resolution elements wide. This corresponds to approximately 25-to1 length-to-width ratio as is seen in the reconstructed figure. 


\section{3\% ENRICHED PIN}

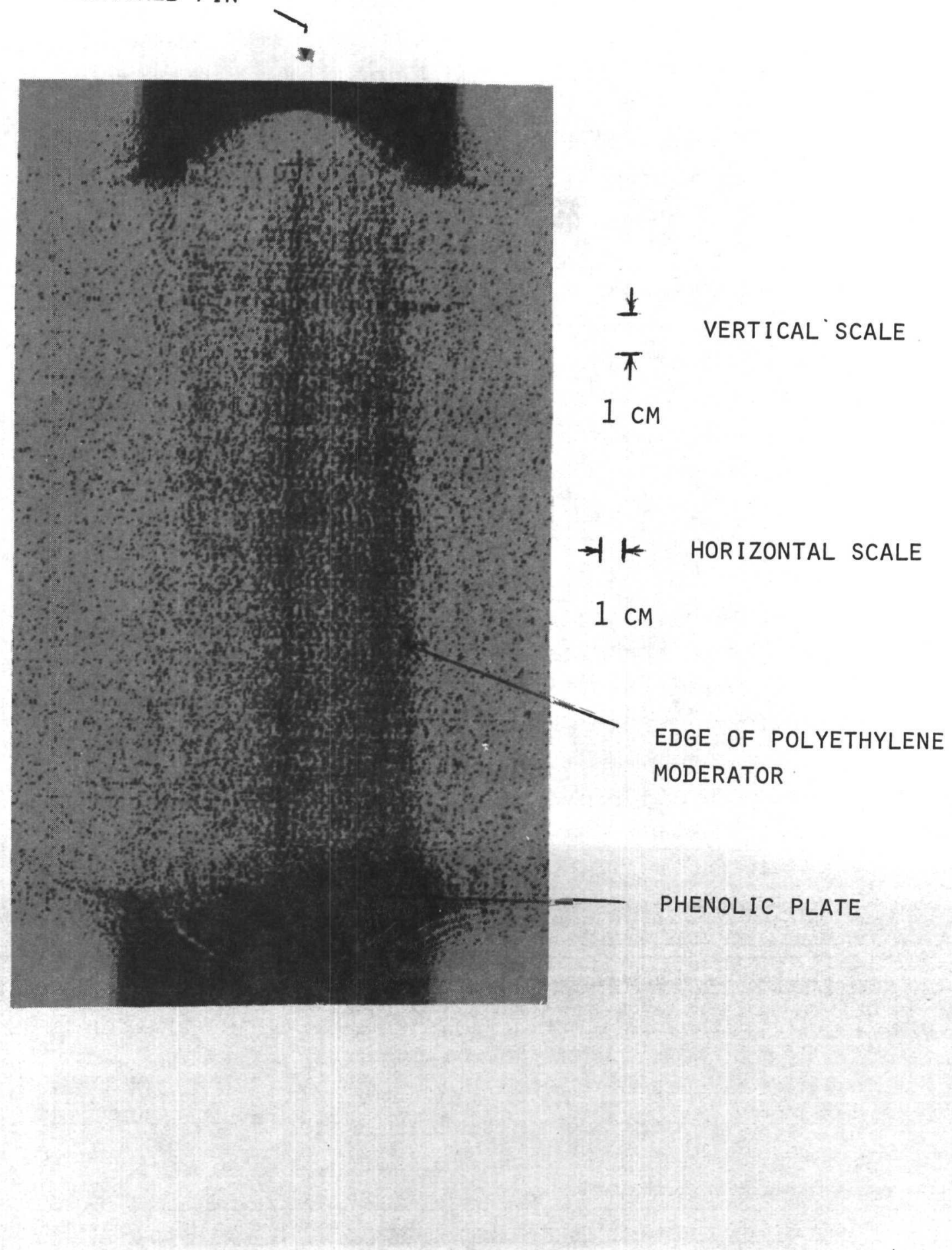

Figure 29. Computer Reconstruction of a 93\% Enriched Pin Imaged at SPR II (Note: The vertical and horizontal scales are different.) 


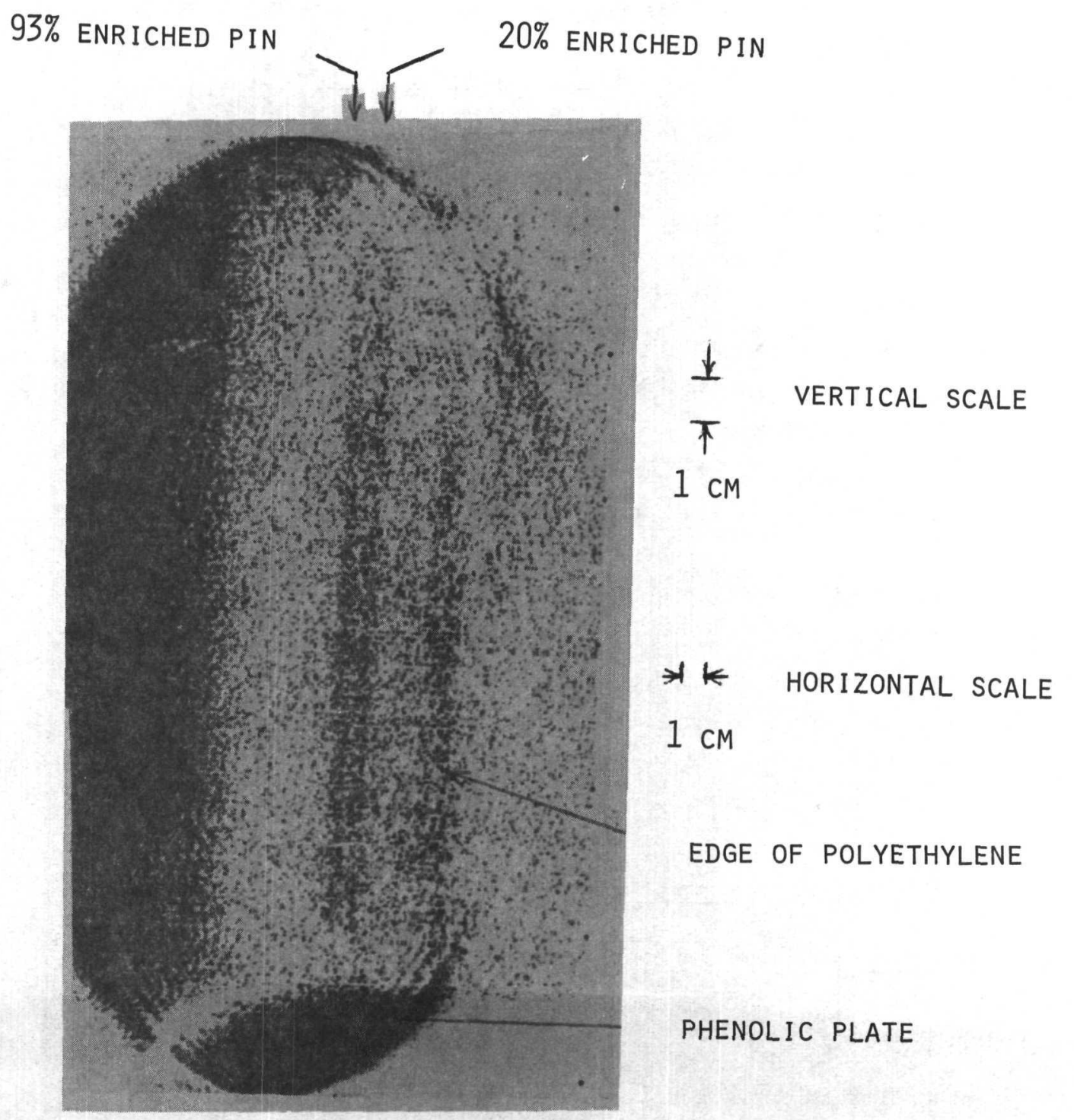

Figure 30. Computer Reconstruction of a 93\% Enriched Pin and a 20\% Enriched Pin at SPR II (Note: The vertical and horizontal scales are different.) 
The reconstruction in Figure 30 corresponds to the two pin image of Figure 28b. The reconstruction shows the two fuel pins to be clearly visible with one at a slight angle to the other as was the case in the actual experimental situation. In this case, the original scan was done on a 512 x 512 format so the horizontal and vertical scale factors are very nearly the same $\left[\left(R_{1}\right)^{2}=500\right.$, $\mathbb{N}=51[]$. In this case the fuel pins should each be about 16 densitometer resolution elements wide. Because the original pseudohologram was off-center on the output phosphor of the X-ray intensifier, the reconstruction appears off-center in the output array. Also a line artifact is seen on the left as in the previous reconstructed image.

Future work in this area will be directed toward identifying the cause of the artifacts present in these reconstructions and eliminating them if possible. Consideration will also be given to the effect of the nonlinearity introduced during the pseudohologram formation due to the output phosphor and photographic recording characteristics. Finally, in conjunction with Prof. H. H. Barrett of the University of Arizona, nonlinear reconstruction methods to increase image-to-background ratios are being studied.

\subsubsection{Scintillator Investigations}

Quality pseudoholograms from which good images can be reconstructed must be composed of an adequate number of detected photons from each resolution element (pixel) of the object. If this is not the case, then the image will be degraded by quantum limitations. For image recording with $\gamma$-rays, the statistics are determined by the number of photons from each pixel which interact with the scintillator (not by the number of optical photons collected by the recording system). Therefore, the scintillator should be as thick as possible, consistent with the requirements of spatial resolution. If the Compton electrons generated in the crystal did not spread the region over which the energy supplied by the incident $\gamma$-ray is deposited and if there were not internal light scattering effects in the crystals, the thickness would only be limited by the requirement that most photons interact only once. It was clear in the early planning that the 
Compton effect would probably limit scintillator thicknesses to less than $1 \mathrm{~mm}$ if $1 \mathrm{~mm}$ resolution in the imaging had to be obtained, but the potential importance of the light scattering in the scintillator was not appreciated.

The first thin NaI crystals we obtained were polycrystalline to provide greater strength for the large $20-\mathrm{cm}$ diameter required. They had a blackened .13-mm Ta layer on the $\gamma$-ray entrance side and a flat quartz optical face plate behind. It has been found, however, that the black surface is not optically coupled properly to the crystal, and light is scattered severely from the unpolished crystal surface on that side. Figure 31 shows the geometry of the scintillator and the manner in which a laser beam was used to investigate light scattering effects. The beam was about $.05 \mathrm{~mm}$ in diameter but at the rear surface it made a spot that was at least $.15 \mathrm{~mm}$ in diameter. We believe that part of this spread comes when the laser beam enters the crystal, but that much of it comes from multiple reflections in the crystal and scattering from the rough rear surface. In fact, a significant amount of light can be seen emerging from points $2 \mathrm{~cm}$ away from the entry position. This problem is not expected to be cured easily by simply using a thinner crystal. Instead, another geometry will be investigated. Ideally, if the two surfaces of single crystal are highly polished, then light generated inside at high enough angles to the normal to miss the external light gathering optics $\left(>4^{\circ}\right)$ will never emerge within that light cone and won't be collected as background. If there is a good optical match between the crystal and the glass, only a few internal reflections will be made for normal rays. Therefore, the back surface reflected light will not be displaced laterally enough to degrade resolution.

We are also investigating another scintillator design which could increase the sensitivity by 10 and, in addition, the modulation transfer function at $4 \mathrm{lp} / \mathrm{mm}$ by another factor of 10. A rough conceptual design is shown in Figure 32. Suppose that .25-mm thick NaI sheets are sandwiched between .12-cm sheets of gold. The light generated in each layer would be isolated from neighboring zones, and the light emerging would be more effectively channeled toward the collecting optics. Further, the average density of the medium would be raised from 3.7 grams/ $\mathrm{cm}^{3}$ to $9 \mathrm{grams} / \mathrm{cm}^{3}$. Its sensitivity to high energy $\gamma$-rays would thereby be increased. 


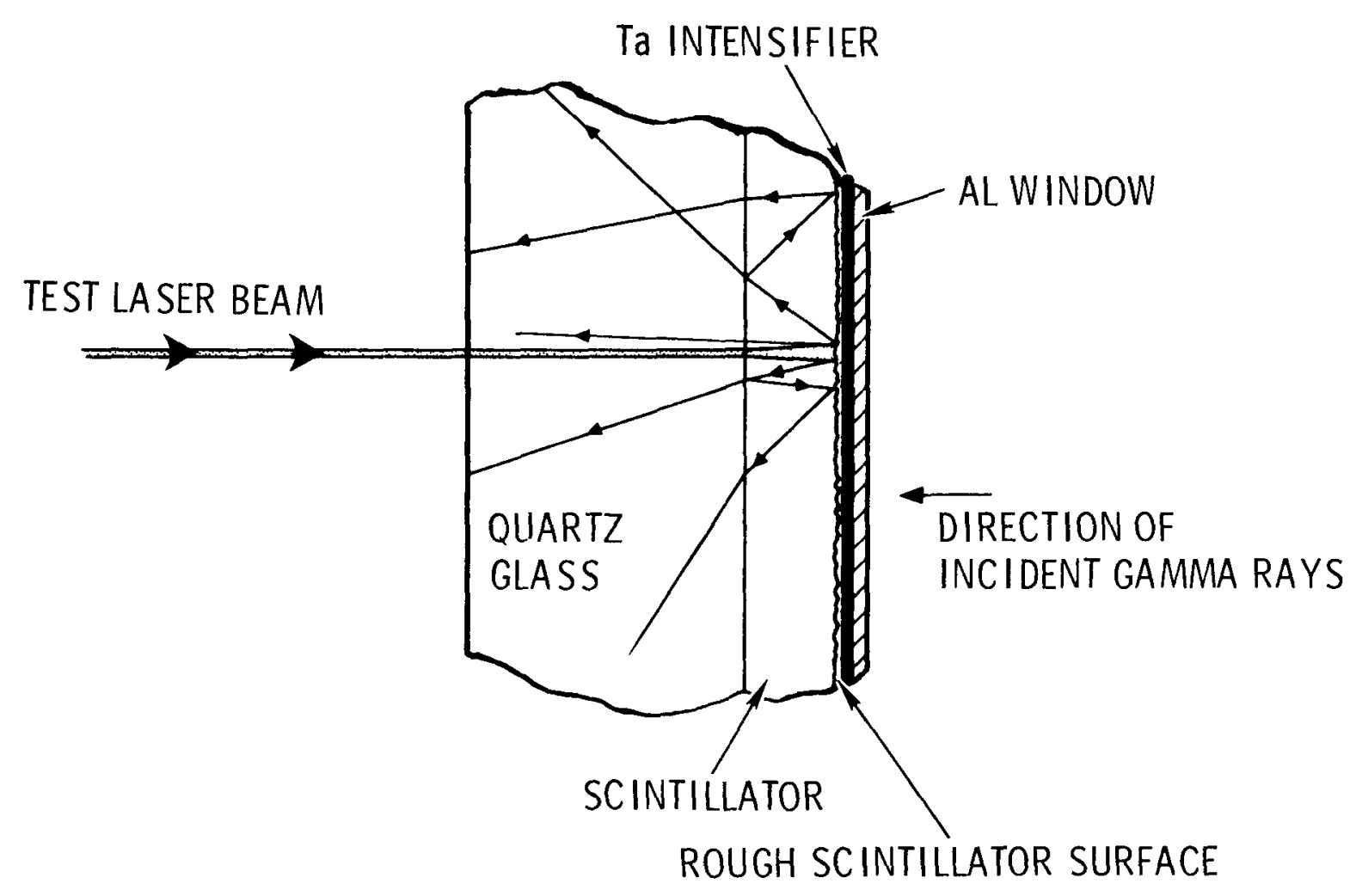

Figure 31. Scintillator Geometry and Laser Light Scattering Modes 


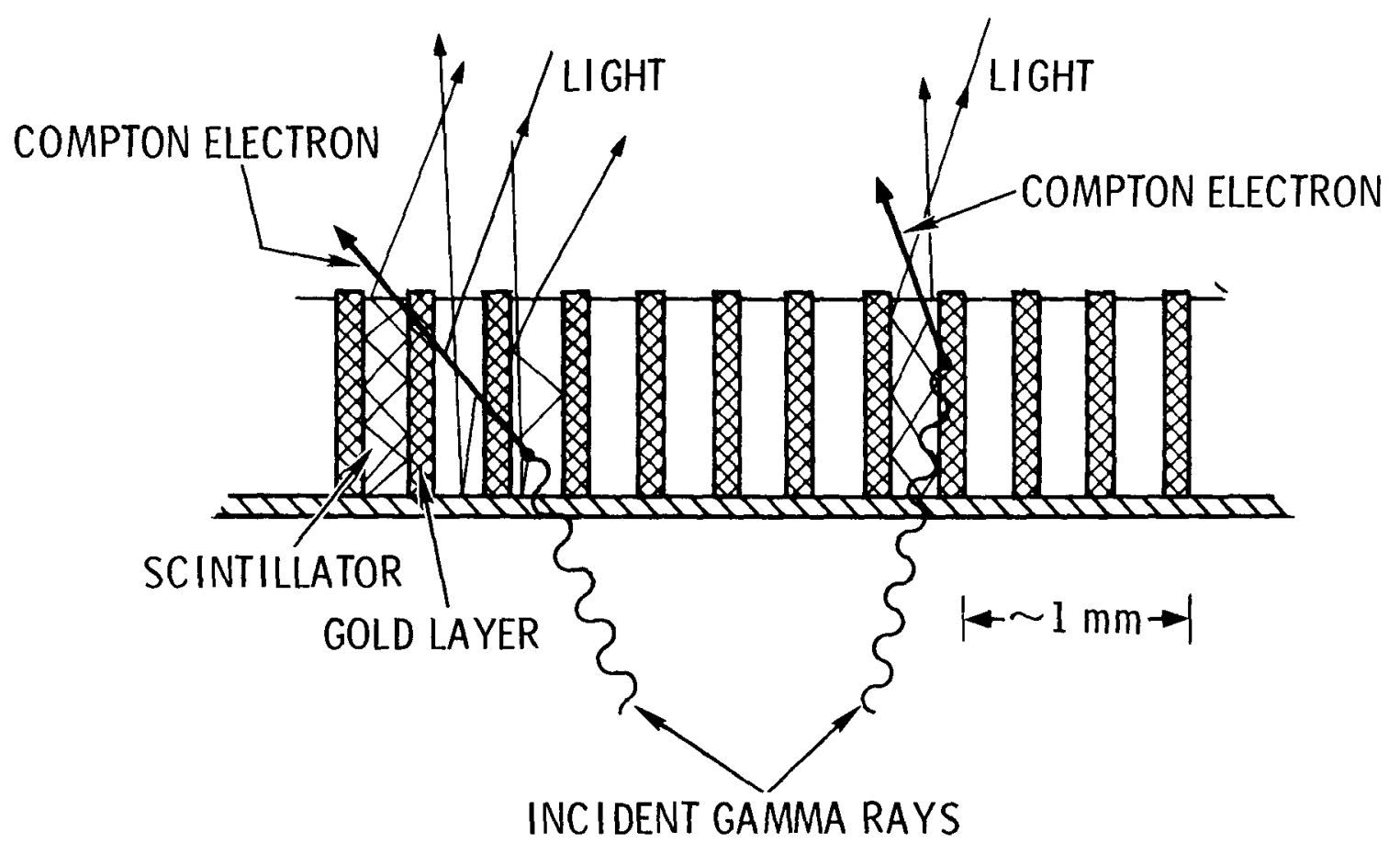

Figure 32. Layered Scintillator Construction 
We have found that a small test device based on similar ideas has been designed for a 20-keV X-ray source by a company in California. The zones were .075-mm thick CsI sheets sandwiched between .025-mm layers of gold. It was fabricated in 1975 but has never been tested. Arrangements are now being made to test the device at Sandia Laboratories.

\subsubsection{The Collimation Test Module}

We have constructed a collimation and shielding test module for the ACPR to see if useful signal-to-background ratios can be obtained there. If this is found to be so, it should prove that with proper engineering a viable fuel motion detection system based on coded aperture imaging can be built.

The geometry for this test is shown in Figure 33. The module is mounted beside the reactor on the table which guides the radiography tube. Certain fairly significant compromises had to be made to accommodate this module to the tube; but since it will be removed when the final fuel motion system is installed, we are confident that, if a satisfactory signal-to-noise ratio can be obtained here, it will be relatively much easier to accomplish later with improved geometry and more room.

Eleven fuel rods will be removed from the core to form a slot into which the slot collimator will be inserted. Just outside the core is a 4.5-cm thick sandwich of steel and boral. The steel is an effective attenuator of high energy neutrons because of its high inelastic scattering cross section. Behind it is a tank of $\mathrm{NaBF}_{4}$ in a water solution and a $20-\mathrm{cm}$ wall of $\mathrm{Pb}$. Inside the radiography tube is a lead chamber where the film detector will be placed. (There is not room in the radiography tube for an active system and it is really not necessary at this stage of the program.) Penetrating the midale portion of the shield is a chamber containing the coded aperture and the main collimator. In front of the collimator is a container of $\mathrm{LiH}$ which should significantly reduce the neutron fluence in the beam.

All of these components, except the slot collimator, have now been lowered into position $10 \mathrm{~m}$ down in the water. Each component has alignment devices attached so that, when it contacts the part below, it is automatically guided into 


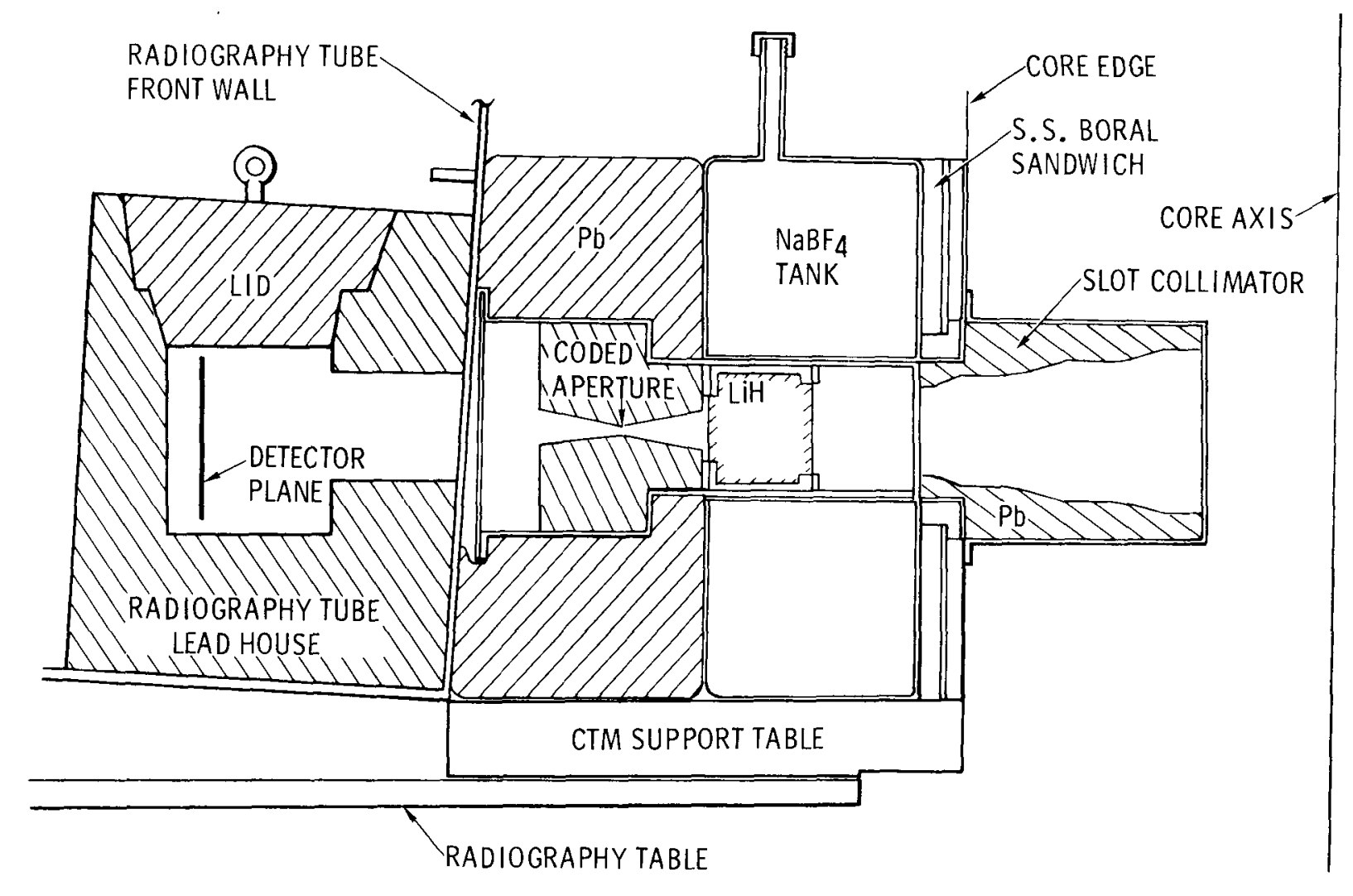

Figure 33. Schematic of the Collimation Test Module 
the exact position. One of the major objectives of this experiment was to test our ability to maneuver and accurately align heavy and awkward components at this depth. Indeed, the assembly procedure turned out to be easier than expected.

Shielding calculations for this type of layered neutron-gamma ray shield have been carried out with a Monte Carlo code "Morse." The calculations indicate that the shield should be very effective in stopping the primary core $\gamma$-rays, the lower energy neutrons, and the secondary $\gamma$-rays generated in front of the lead. The principal background at the detector location (neglecting that transmitted by the collimator) will be from inelastic $\gamma$-rays generated in the rear portion of the lead by penetrating high energy neutrons. For this module more shielding could not be added because of space limitations, but in the final shield an additional $15 \mathrm{~cm}$ of steel may be necessary.

\subsubsection{Signal-to-Background Estimates}

Until the Collimation Test Module proves that an adequate signal-to-noise level can be obtained in the ACPR geometry, it will not be known for certain that the fuel motion system will provide the required information. Signal-to-background estimates have therefore been made. They indicate to us that the measurements should be successful.

The first order estimate is based on the geometry shown in Figure 34. The fuel pin is observed through the slot collimator by a detector. We assume that there is no scattering or penetration of the collimator edges. This calculation will supply relative source intensities of the fuel pin and the wall behind it. Therefore, the collimator in this case will only serve to assure good geometry (exponential attenuations) for the primary $\gamma$-rays. It will also be assumed that, on the average, the fission $\gamma$-ray spectrum can be approximated by a beam of 1-MeV photons which have a total average attenuation coefficient of $\mu=.08 \mathrm{~cm}^{2} / \mathrm{gram}$. If we also assume that the thermal neutron flux in the central region of the core is uniform, then the source strength, $I_{0}$ either in the fuel pin or in the wall will be proportional to the ${ }^{235} \mathrm{U}$ density.

$$
I_{0}=A p_{u}
$$




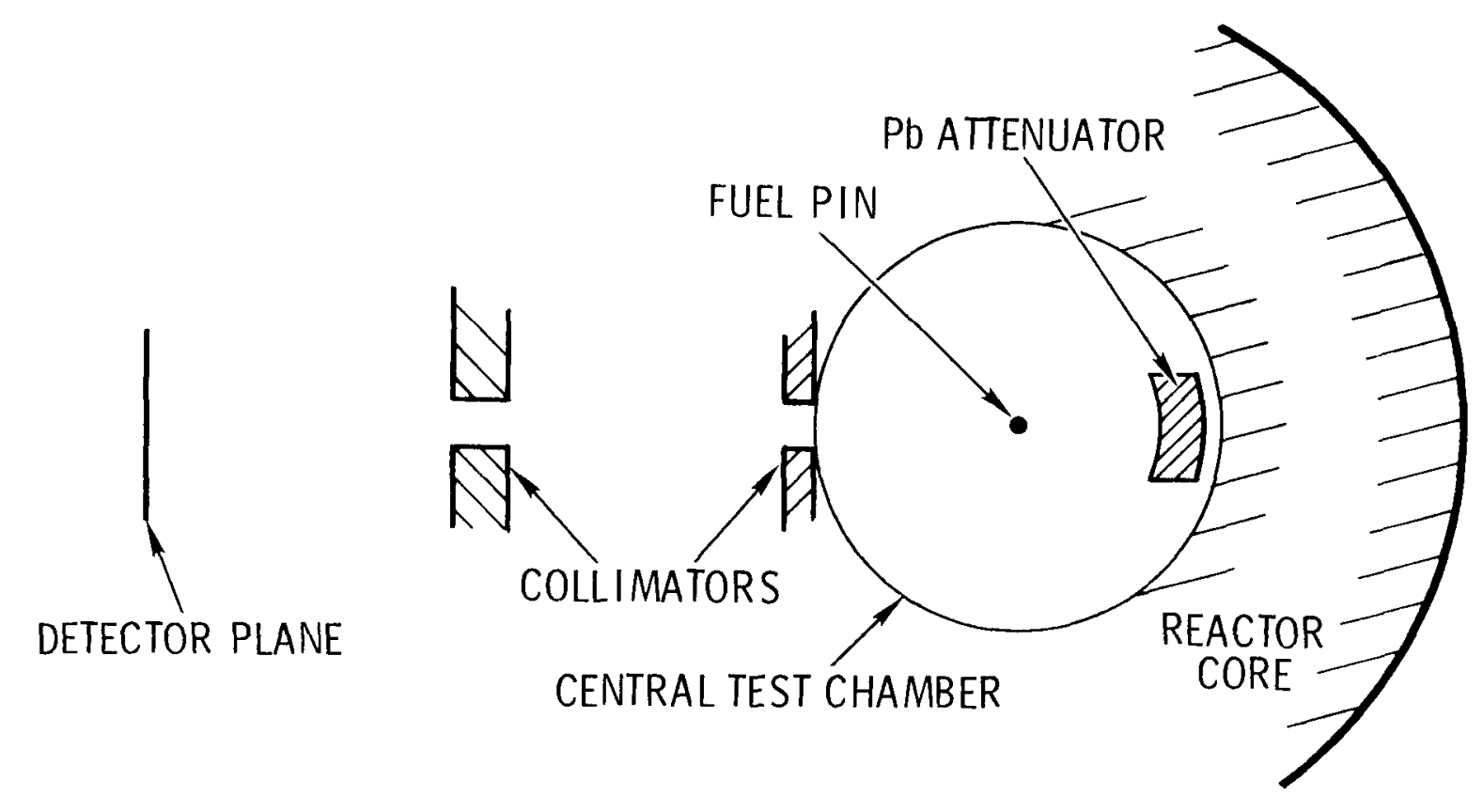

Firure 34. Feometry for Signal-to-Background Ratio Fistimate 
where $\rho_{u}$ is the uranium density in grams $/ \mathrm{cm}^{3}$ in the material and $A$ is a factor which tells us what fraction of these source $\gamma$-rays emerge from the material in a direction such that they will be transmitted by the collimator. Now, if the lead block in Figure 32 is not in place and if we assume that the wall behind is an infinite thickness of driver core fuel of density, $\rho_{1}$, the intensity of radiation emerging from the driver core wall and directed toward the collimator will be

$$
\begin{aligned}
I_{I} & =A \rho_{u} \int_{\sigma}^{\infty} e^{-\mu \rho_{1} x} d x \\
& =\frac{A \rho_{u}}{\mu \rho_{I}}
\end{aligned}
$$

Next, we assume that this beam is attenuated by an $x_{2}$ thickness of lead with density $\mathrm{P}_{2}$. The background intensity then becomes

$$
I_{B}=\frac{A p_{u}}{\mu p_{1}} e^{-\mu p_{2} x_{2}}
$$

The pin source intensity can be found in the same manner and, in this geometry, the A factors should be about the same, I'。=A $\rho^{\prime}{ }_{u}$. If this is integrated over a pin diameter, we find

$$
\begin{aligned}
I_{S} & =I_{0} \int_{0}^{D} e^{-\mu \rho_{3} x} d x \\
& =\frac{A \rho^{\prime} u}{\mu \rho_{3}}\left[1-e^{-\mu \rho_{3} D}\right] .
\end{aligned}
$$

In the driver core, the fuel is $100_{2}$ BeO with a density of $\rho_{1}=3.5 \mathrm{~g} / \mathrm{cm}^{3}$. The uranium is $20 \%$ by weight and is $35 \%$ enriched so that $\rho_{u}=\rho_{I}(.20)(.35)=.245 \mathrm{~g} / \mathrm{cm}^{3}$. Also, take $x_{2}=3.0 \mathrm{~cm}$, and $\rho_{2}=11.3 \mathrm{~g} / \mathrm{cm}^{3}$. Substituting in these numbers, the background intensity is found to be 


$$
\begin{aligned}
I_{B} & =\frac{A(.245)}{(.08)(3.5)} \mathrm{e}^{-(.08)(11.3)(3)} \\
& =.058 \mathrm{~A} .
\end{aligned}
$$

For the fuel pin, which is $88 \%$ uranium by weight and $15 \%$ enriched, the ${ }^{235} \mathrm{U}$ density

$$
\begin{gathered}
\text { is } \rho_{\mu}=\left(10.9 \mathrm{~g} / \mathrm{cm}^{3}\right)(.88)(.15)=1.44 \mathrm{~g} / \mathrm{cm}^{3} \text {. Also, } \rho_{3}=10.9 \mathrm{~g} / \mathrm{cm}^{3}, D=.5 \mathrm{~cm}, \\
I_{S}=\frac{A(1.44)}{(.08)(10.9)}\left[1-\mathrm{e}^{-(.08)(10.9)(.5)],}\right. \\
I_{\mathrm{S}}=.93 \mathrm{~A} .
\end{gathered}
$$

Therefore, the ideal signal-to-noise ratio is

$$
\frac{I_{S}}{I_{B}}=17
$$

Many important factors have been left out of this estimate. No consideration has been given to buildup of scattered radiation through the lead, to backscattering of core $\gamma$-rays from the lead surface, or from neutron induced inelastic $\gamma$-rays. Still the simple estimate provides a hopeful upper limit to the obtainable SNR.

To investigate the other contributions to background from buildup and scattering in the lead, a coupled electron photon Monte Carlo code called Tiger was run in the geometry shown in Figure 35. A uniform source of fission $\gamma$-rays was assumed to be incident from the left on a $2-\mathrm{cm}$ thick layer of lead. The source was isotropic into $2 \pi$ in the forward direction. The calculation showed that only 1.5 percent of the incident photons were backscattered into a cone with a 10 percent half angle to the normal to the lead. This clearly demonstrates that, even without the lead, most of the $\gamma$-ray flux emerging from the wall behind the fuel pin will be primary radiation ( $\gamma$-rays generated by fissions in the fuel and possibly undergoing small angle scatterings in coming out). Therefore, since the lead is not a primary source of fission $\gamma$-rays, it must be to our advantage to place the block behind the pin. the primaries will be attenuated by a factor of 10, and the backscattered component will be about the same for the lead or for the rear wall. There will be a buildup 


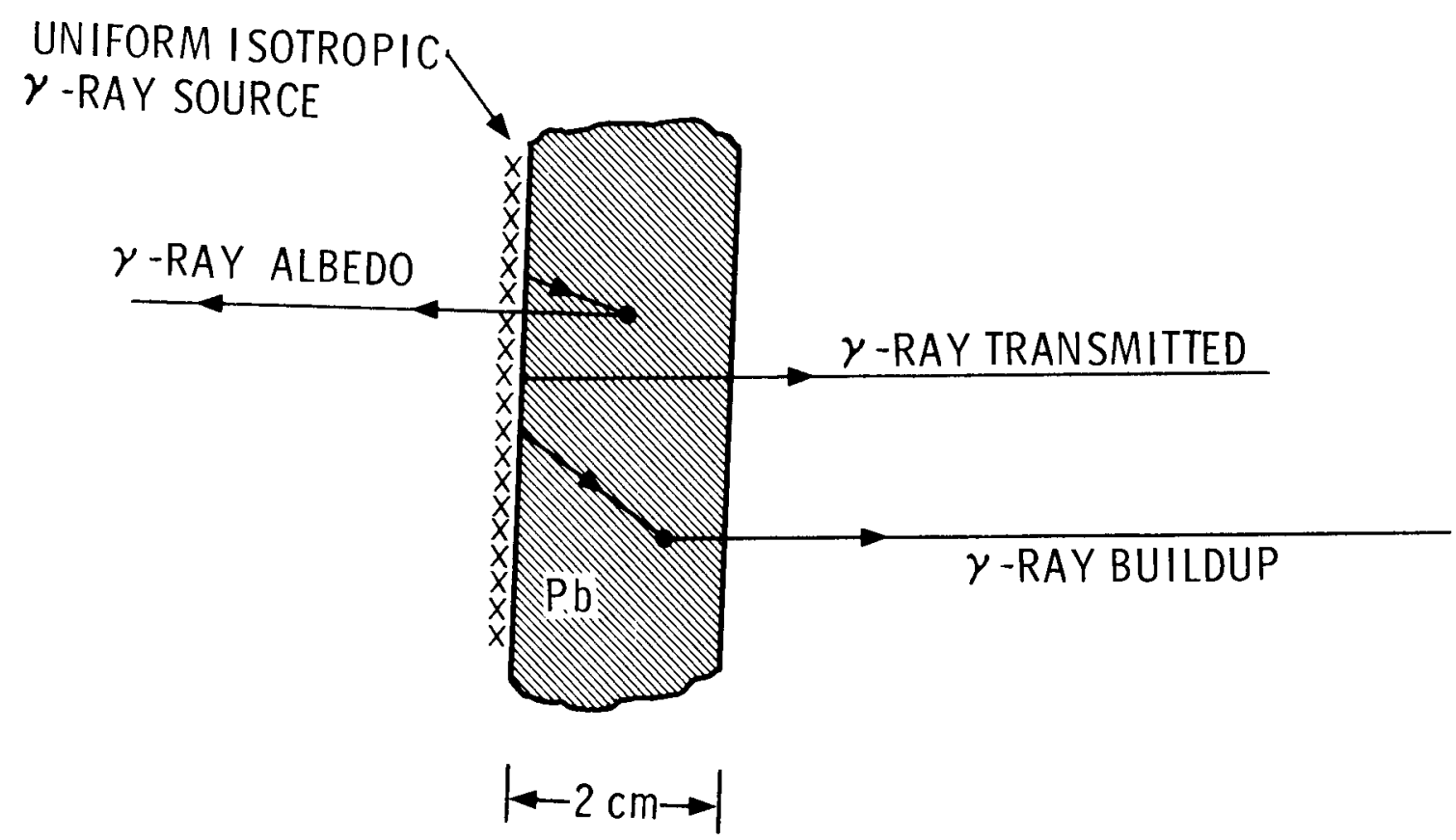

Figure 35. Geometry for Estimate of $\gamma$-Ray Albedo and Transmitted Flux from a Lead Layer 
factor for the primaries, however, as they pass through the lead (some photons are scattered into the beam). From the same code, the buildup was found to be only about 2 percent for scattering into the same 10 degree half angle cone for the $\gamma$ beam transmitted through the lead. These calculations simply mean that, if a few $\mathrm{cm}$ of lead is placed between the wall and the fuel pin, the primary r-radiation (which composes more than 90 percent of the photon radiation emerging from the wall) will be attenuated down by about a factor of 10 to the levels of backscattered and inscattered radiation (buildup). The fuel pin will therefore be considerably brighter than the background. Our analyses also show that, even if the lead block were removed, the fuel pin would be brighter than the background. Since the coded aperture allows us to geometrically separate signals and background, there would still be enough modulation in the pseudohologram to allow imaging. Another way to visualize this is to recognize that the signal convolved with the aperture has a different structure than the background convolved with the aperture. Consequently, these structures superimpose in the pseudohologram and reconstruct separately. They do not cancel or destroy each other.

The final major source of $\gamma$-rays could come from neutrons scattering inelastically in the lead. Another simple estimate has indicated that at the detector plane the flux of these inelastic $\gamma$-rays will be an order of magnitude lower than the signal flux. More careful calculations have not yet been made. As mentioned before, final resolution of this question awaits the result of the CTM experiment.

\subsection{Fission Track Fuel Motion Monitor System}

Certain dielectrics have the property that, when they are struck by an energetic heavy ion, the track taken by the ion is more susceptible to etching than is the undamaged bulk material. When such dielectrics are exposed to a mixture of radiations, including fission fragments, and are then etched, the fission fragment tracks become craters while the dielectrics show no signs of exposure to the other radiations. These background-insensitive dielectrics form the basis of a fuel motion monitor system now being studied for use on the ACPR upgrade. The system will view fast neutrons from the test fuel through a multichannel collimator. ${ }^{238} \mathrm{U}$ (or $237 \mathrm{~Np}$ ) at the exits of the collimator channels will convert these neutrons into fission fragments which will be registered on a dielectric tape (probably mylar) Iocated less than $1.3 \mathrm{~mm}$ behind the uranium. The etched tape will then produce an image of the neutron source, i.e., the test fuel. 
The essentials of the system may be seen in Figure 36. The view in the upper left of this figure looks down on the system. In this view the multichannel collimator extends to the left. Time resolution is to be achieved by transporting the tape past the collimator channels in such a way that no segment of tape views more than one collimator channel during the reactor burst. This is achieved by moving the tape in the zig-zag fashion seen in the view shown at the lower left of Figure 36 , and by using a shutter, indicated by the dots between the collimator and tape assembly, to isolate the tape from the uranium at the end of the pulse. The remaining view in Figure 36 looks towards the reactor core, and this view shows the tape recorder that will be used to move the tape, and the roller assembly mounted on the tape recorder.

Figure 37 is a photograph of the rolier assembly. The assembly is $57 \mathrm{~cm} \mathrm{high,}$ and it includes 71 pairs of $.64-\mathrm{cm}$ diameter rollers. These rollers, with a centerto-center spacing of $.79 \mathrm{~cm}$, provide 71 vertical views of the test fuel region. At each vertical level there are seven $1.59 \mathrm{~mm} \times 1.59 \mathrm{~mm}$ collimator channels across the $2.54-\mathrm{cm}$ width of the collimator.

This photograph was taken shurtiy after the ruller assembly was received srom oun shops. The partial tape loading, shown in the photograph, reveals a design defect that has since been rectified. The tape was unable to pass smoothly through the sharp turn that resulted from the location of the rear roller (lower right of photograph). These rollers have since been moved (to the left in the photograph) and the tape now transports properly. To date, with rollers twice the diameter of those shown in Figure 37, utilizing half of the rollers, the tape has been transported without difficulty at $305 \mathrm{~cm} / \mathrm{s}$. With collimator channels whose vertical dimensions are $1.59 \mathrm{~mm}$, this implies that time resolution of the order of $0.5 \mathrm{~ms}$ is achievable. Rollers of twice the size that will ultimately be used in this system have the effect of increasing the vertical sampling interval to $1.59 \mathrm{~cm}$. These rollers were used because modifications are necessary to make the tape transport adequately over the smaller rollers. These modifications, which include an advanced design roller shape and nylatron bearing surfaces, are expected to permit use of the smaller rollers at full speed.

With confidence now in our ability to transport the dielectric tape in the prescribed manner, our attention will now focus on design and fabrication of a relatively simple collimator that will be compatible with the ACPR upgrade geometry. 

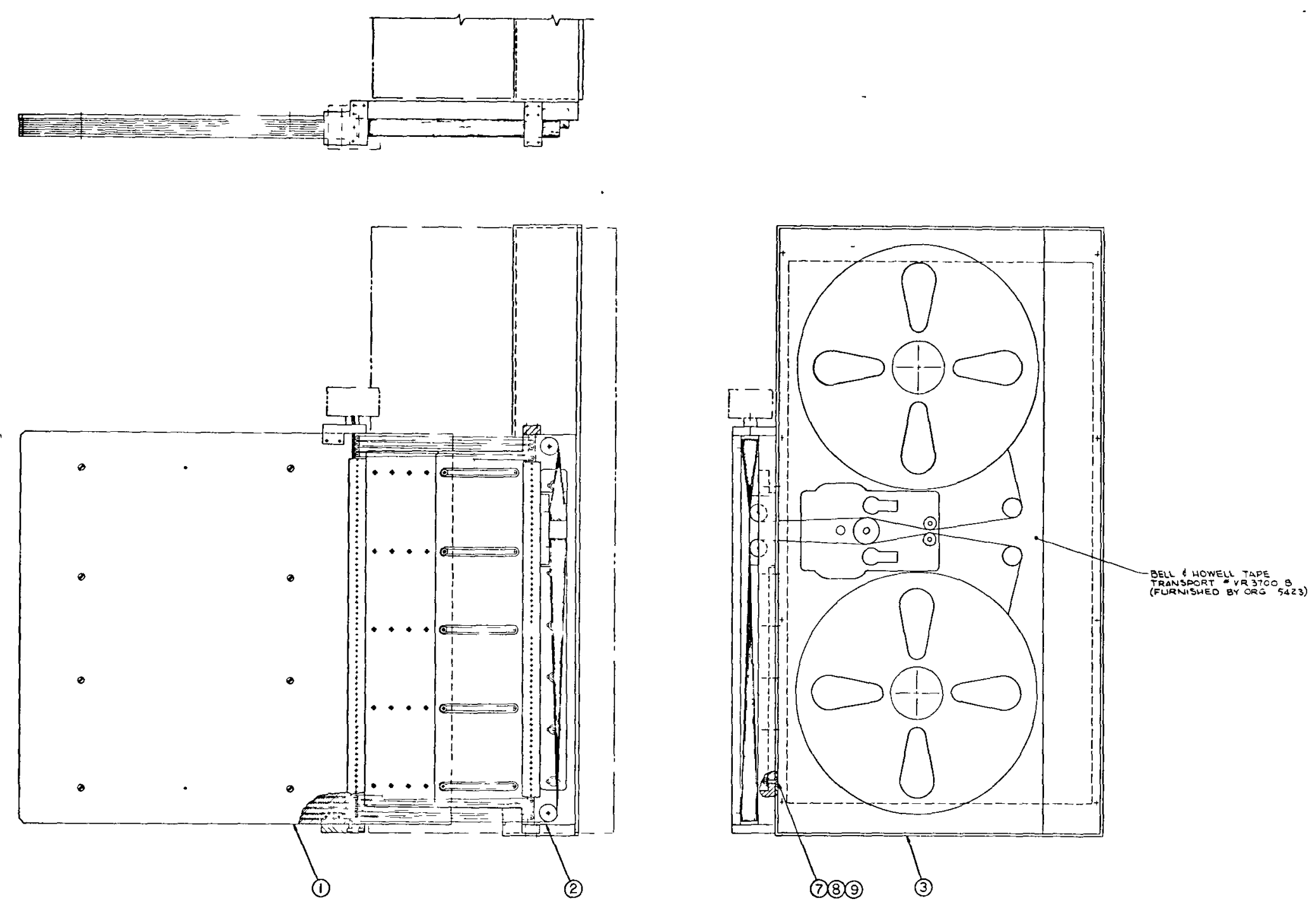

Figure 36. Fuel Rod Motion Detector Assembly 


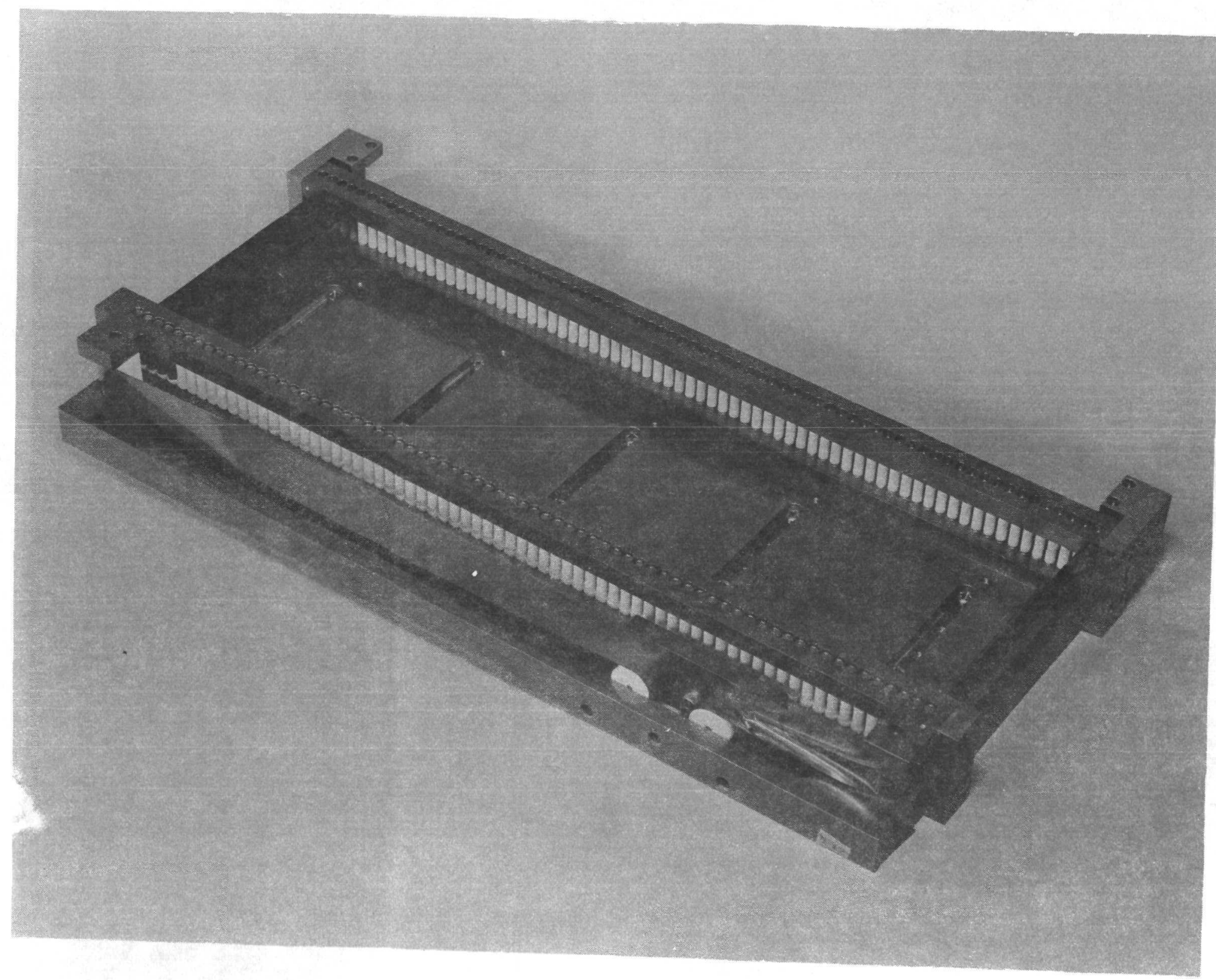

Figure 37. Photograph of Roller Assembly 
Concurrent with development of the system that will produce the fission fragment tracks that bear the time history of fuel motion, consideration has been given to methods of reading these tracks. In preliminary work, it appeared that using alpha particles as probes to measure the track density of fission fragments on the dielectrics was feasible. 28 There remained, however, some question about whether this reading technique would work in other than ideal circumstances. Ideal circumstances include a relatively large fission track density $\left(\gtrsim 10^{5} \mathrm{~cm}^{2}\right)$, fission craters that extend all the way through the dielectric, a nondegraded fission fragment spectrum, and fission fragments that are normally incident on the dielectric. Most of these circumstances will not be present in our application. The fission track densities will be of the order of $10^{3} \mathrm{~cm}^{2}$; the 1-mil thick mylar we will probably use is greater than the range of fission fragments and so the craters will not extend all the way through the dielectric; and, with only 1.3-mm spacing between the uranium and dielectric, the fragments will not in general be normally incident. Furthermore, to eliminate calibration differences between channels, a thick $\left(\gtrsim 5 \times 10^{-3} \mathrm{~g} / \mathrm{cm}^{2}\right)$ uranium deposit will be used, and this will produce a degraded fission fragment spectrum.

To settle these questions, a series of experiments has been performed, the net result of which is that the reading technique will work as hoped. All of these experiments used fission fragments from a bare ${ }^{252} \mathrm{Cf}$ source to produce the fission fragment damage in the dielectrics.

In the first experiment, samples of Kimfoil were exposed in good geometry (source-Kimfoil separation $\geq 20.3 \mathrm{~cm}$ ) to produce track densities ranging from less than $10^{3} \mathrm{~cm}^{2}$ to more than $10^{6} \mathrm{~cm}^{2}$. Alpha particles from the decay of ${ }^{244} \mathrm{Cm}$ $(5.8 \mathrm{MeV})$ were then used to read these samples. Figure 38 shows a log display of the spectrum of alpha particles transmitted through a sample with a track density of $9.8 \times 10^{2} \mathrm{~cm}^{2}$ in the foreground. The spectrum of alphas transmitted through the sample with $1.1 \times 10^{6}$ craters $/ \mathrm{cm}^{2}$ is in the background. Using the prescription given in Reference 28, in which a spectrum with known track density is used to normalize an unknown spectrum, the track densities for the "unknown" samples were found to be in good agreement with the known track densities to which the samples were exposed.

The next experiment was designed to investigate effects due to degradation of the fission fragment energy spectrum. A sheet of $1.27 \times 10^{-3} \mathrm{~cm}$-thick aluminized 


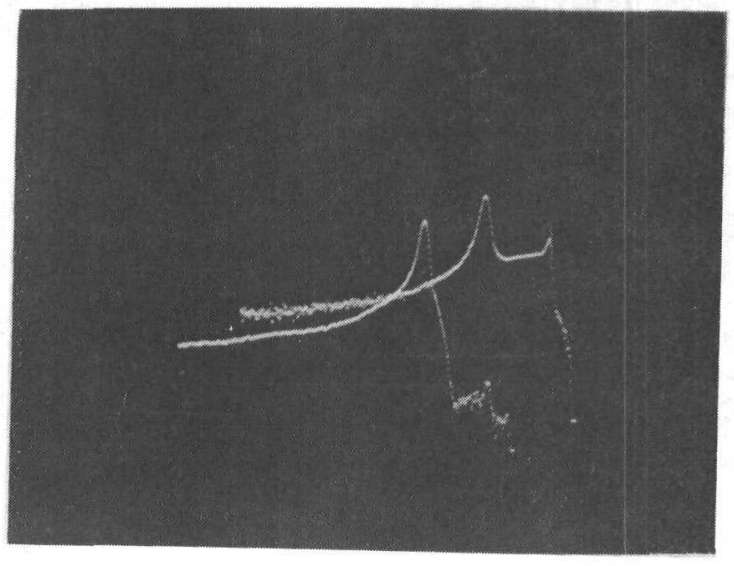

Figure 38. Log Display of Spectrum of Alpha Particles Transmitted through a Sample with a Track Density of $9.8 \times 10^{2} \mathrm{~cm}^{2}$ in the Foreground, and through a Sample with $1.1 \times 10^{6}$ Craters $/ \mathrm{cm}^{2}$ in the Background

Figure 39. Photograph of the Sample Exposed with $0.127 \mathrm{~cm}$ Source-Sample Separation

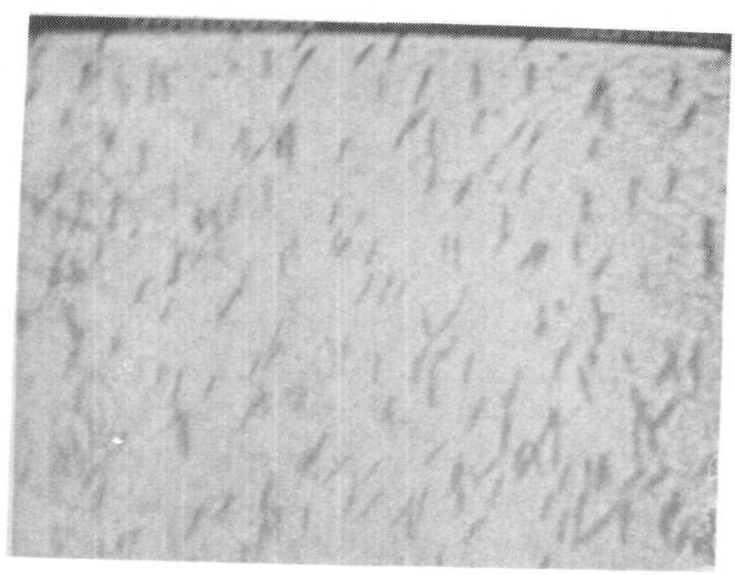

Figure 40. Same as Figure 39 - Separation $7.62 \mathrm{~cm}$ 
mylar was placed between the ${ }^{252} \mathrm{Cf}$ fission fragment source and the Kimfoil that was to be exposed. While it was feared that a large fraction of the fission fragments would have insufficient energy to produce craters, it was found this was not the case. Essentially all of the fragments were energetic enough to produce craters. The only effect was a slight reduction in the size of each of the craters.

In the next experiment, both the effects of using a thick dielectric (2.54 $\mathrm{x}$ $10^{-3} \mathrm{~cm}$ ) and of using mylar as the dielectric were studied. The mylar used was identical to that which is used in tape recorders except it bore no oxide coating. The tape was exposed, in good geometry, to undergraded fragments from ${ }^{252} \mathrm{Cf}$. When the tape was etched, it was found that good quality craters had been produced. These craters were studied using both optical reading techniques and the alpha particle probe technique.

In the final experiments the effects of nonideal geometry were studied. Kimfoil was exposed to the fragments from ${ }^{252} \mathrm{Cf}$ with a source-sample separation of only $.127 \mathrm{~cm}$. Figure 39 shows a photograph of a magnified view of the sample that was exposed with the close spacing, and Figure 40 shows the craters in a sample that was $7.62 \mathrm{~cm}$ from the source when it was exposed. The difference between the angles the craters make with the sample surface in the two cases is striking, and yet optical analysis shows the close proximity resulted in no loss of craters. This experiment also demonstrated that, with a simple adjustment for average crater area, the technique given in Reference 28 works well even with samples with craters as dissimilar as these. In practice, when a very long tape has been exposed in its ACPR application, the crater areas are easily obtained by using optical techniques to read one small area of the tapes. The remainder of the tape will be read in an automated system that used alpha particle transmission. 
REFERENCES

1. Experimental Fast Reactor Safety Research Program - (Combined) Quarterly Report, July-December 1974, Simulation Sciences Research Department, SAND75-0068, Sandia Laboratories, Albuquerque, New Mexico, February 1975.

2. Experimental Fast Reactor Safety Research Program - Quarterly Report, January-March 1975, Simulation Sciences Research Department, SAND75-0225, Sandia Laboratories, ATbuquerque, New Mexico, Apri 1975.

3. Experimental Fast Reactor Safety Research Program - Quarterly Report, Apri1-June 1975, Simulation Sciences Research Department, SAND75-0449, Sandia Laboratories, Albuquerque, New Mexico, August 1975.

4. Experimental Fast Reactor Safety Research Program - Quarterly Report, July-September 1975, Reactor Development and Research Department, SAND75-0567, Sandia Laboratories, Albuquerque, New Mexico, December 1975.

5. Fast Reactor Safety Research Program - Quarterly Report, October-December 1975, Reactor Research and Development Department, SAND76-0157, Sandia Laboratories, Albuquerque, New Mexico, Aprit 1976.

6. Fast Reactor Safety Research Program - Quarterly Report, January-March 1976, Reactor Research and Development Department, SAND76-0273, Sandia Laboratories, Albuquerque, New Mexico, JuTy 1976.

7. Fast Reactor Safety Research Program - Quarterly Report, April-June 1976, Reactor Research and Development Department, SAND76-0372, Sandia Laboratories, ATbuquerque, New Mexico, September 1976.

8. Fast Reactor Safety Research Program - Quarterly Report, July-September 1976, Reactor Research and Development Department, SAND76-0652, Sandia Laboratories, Albuquerque, New Mexico, January 1977.

9. Fast Reactor Safety Research Program - Quarterly Report, October-December 1976, Reactor Research and Development Department, SAND77-0145, Sandia Laboratories, A7buquerque, New Mexico, March 1977.

10. G. C. Benson, P. I. Freeman, E. Dempsey, Amer. Ceram. Soc. 46, 43(1963).

11. J. H. Henke1, J. Chem. Phys. 23, 681(1955).

12. G. Dolling, Can. J. Phys. $\underline{43}, 1397(1965)$.

13. D. A. Benson, L. D. Posey, K. 0. Reil, T. R. Schmidt, A. L. Ouellette, W. H. Buckalew, R. J. Haushalter, Experimental Fast Reactor Safety Research Program - Quarterly Report, April-June 1975, Chapter 4, SAND75-0449, Sandia Laboratories, A7buquerque, New Mexico, August 1975.

14. J. V. Beck, "Surface Heat Flux Determination Using an Integral Method," Nuclear Engineering and Design 7, 170(1968).

15. R. W. Ohse, P. G. Berrie, H. G. Bogensberger, E. A. Fischer, "F ision of Vapor Pressure Measurements of Nuclear Fuels $(U, \mathrm{Pu})_{2}$ and $\mathrm{UO}_{2}$ to $\rightarrow$ ir Fast Reactor Safety Analysis," Journal of Nuclear Materials 59, 112?:: 
References (cont'd):

16. M. Bober, H. U. Karow, K. Schretzmann, "Evaporation Experiments to Determine the Vapor Pressure of $\mathrm{UO}_{2}$ Fuel (3000-5000 K)," Thermodynamics of Nuclear Materials, 1974, Internationa 1 Atomic Energy Agency, Vienna (1975).

17. D. A. Benson, W. H. Buckalew, "The Uranium Dioxide Vapor Equation of State," ANS Transactions $\underline{23}, 325(1976)$.

18. J. H. Scott, et al., MEDL-TME 75-9, Hanford Engineering Development Laboratory, Richland, Washington, October 1974 .

19. R. W. Ostensen, reported upon elsewhere in this quarterly progress report.

20. D. W. Varela, J. S. Philbin, Temperature Capability for DTSIV (DTF-71) version), SAND76-0742, Sandia Laboratories, Albuquerque, New Mexico, to be published.

21. N. M. Green, et al., AMPX: A Modular Code System for Generating Coupled MultiGroup Neutron-Gamma Libraries from ENDF-B, ORNL/TM-3706, Oak Ridge Nationa T Laboratory, Oak Ridge, Tennessee, March 1976.

22. R. Avery, et al., ANL Findings and Recommendations on LMFBR Safety Test Needs and Acquisition of New In-Pile Testing Facilities, ANL/RAS 74-23, Argonne Nationa 7 Laboratory, Argonne, I11inois, March 1975.

23. Light Water Reactor Safety Research Program Quarterly Report January-March 1976, D. A. Dahlgren, Ed., SAND76-0369, Sandia Laboratories, Albuquerque, New Mexico, August 1976.

24. K. D. Lathrop, F. W. Brinkley, Theory and Use of the General-Geometry TWOTRAN Program, LA-4432, Los ATamos Scientific Laboratory, Los Alamos, New Mexico, 1970.

25. J. B. Conway, "An Assessment of the Effect of Long Hold Periods on Low-Cycle Fatigue Life to Supplement Current High-Temperature Design Criteria," an unsolicited proposal from Mar-Test, Inc., Cincinnati, Ohio, August 1976.

26. K. F. Hale, "Creep Failure Prediction from Observation of Microstructure in $2-1 / 4 \% \mathrm{Cr}-1 \%$ Mo Stee 1," Phys ical Metallurgy of Reactor Fuel Elements, Proceedings, Internationa 7 Conference September 2-7, 1973, The Metals Society, 1 Carlton House Terrace, London, SW7Y 5DB, England.

27. K. R. Hessel, K. T. Stalker, "Computer Reconstruction of Pseudoholograms Obtained from a Fission Gamma Source," Transactions of Conference on Fuel and Clad Motion Diagnostics in LMFBR Safety Test Facilities, November 11-12, 1975, SAND76-5547, Sandia Laboratories, ATbuquerque, New Mexico, October 1976.

28. S. R. Dolce, "Reading Dielectric Track Detectors Using Transmitted Particles," IEEE Transactions on Nuclear Science NS-23, No. 1, February 1976. 
DISTRIBUTION:

U. S. Nuclear Regulatory Commission (234 copies for NRC-7)

Division of Document Control

Attn: Steve Scott, Actg. Branch Chief

Distribution Services Branch

Washington, D. C. 20555

Division of Reactor Safety Research (8)

Office of Nuclear Regulatory Research

U. S. Nuclear Regulatory Commission

Washington, D. C. 20555

Attn: C. N. Kelber, Assistant Director, Advanced Reactor Safety Research

M. Silberberg, Chief, Experimental Fast Reactor Safety Branch

R. W. Wright, Experimental Fast Reactor Safety Branch (6)

\section{S. Rubenstein}

Analys is Section

Office of Nuclear Reactor Regulation

Mail Station: Nickelsen Lane

Washington, D. C. 20555

Division of Military Applications (2)

Office of Safety and Facilities

USERDA

Washington, D. C. 20545

Attn: R. T. A. Bredderman

H. M. Busey

L. M. Groover

Program Analys is and Budget

Division of Military Applications

USERDA

Washington, D. C. 20545

Reactor Safety Research Coordination (4)

USERDA

Washington, D. C. 20545

Attn: R. W. Barber, Acting Director (3)

T. E. McSpadden, Project Manager

J. R. Roeder, Director

Operational Safety Division

USERDA/ALO

Albuquerque, New Mexico 87115

Special Programs Division (2)

USERDA/ALO

Albuquerque, New Mexico 87115

Attn: C. B. Quinn

G. W. Johnson

A. DeVolpi

Argonne National Laboratory

9700 South Cass Avenue

Argonne, Illinois 60439 
Distribution (cont'd):

Technical Library (2)

Idaho National Engineering Laboratory

Idaho Falls, Idaho 83401

Attn: James Hunter

J. D. Orndorff

Los Alamos Scientific Laboratory

Group R-5, MS 560

Los A1amos, New Mexico 87545

Robert A. Bari, Group Leader

Safety Evaluation Group

Fast Reactor Safety Division

Brookhaven National Laboratory

Associated Universities Inc.

Upton, Long Is land, New York 11973

University of California

Energy and Kinetics Department

5530 Boelter Hall

Los Angeles, California 90024

Attn: W. E. Kastenberg

Department of Nuclear Engineering

University of New Mexico

Albuquerque, New Mexico 87131

Attn: A. W. Cronenberg

Y. S. Tang

Westinghouse Corporation

Advanced Reactors Division

P. 0. Box 158

Madison, Pennsylvania 15663

B. R. Sehga 1

Electrical Power Research Institute

3412 Hillview Avenue

P. 0. Box 10412

Palo Alto, California 94303

General Electric Corporation (5)

310 De Guigne Drive

Sunnyvale, California 94086

Attn: J. 0. Bradfute, Manager, Dynamics and Safety

A. S. Gibson, Manager, Product Engineering

R. Greebler, Manager, Reactor Studies

J. E. Mott, Senior Engineer, Dynamics \& Safety

M. I. Temme, Manager, Safety Analys is

W. E. Nyer

P. 0. Box 1845

Idaho Falls, Idaho 83401 
Distribution (cont'd):

M. H. McTaggart

MOD(PE) AWRE

Aldermaston

Berkshire

England

J. R. Findley

Building 429

AERE

Harwe11, Didcot

Berkshire

England

A. R. Baker

FRSD

UKAEA

Risley

Warrington

Engl and

T. Ginsberg

Fast Reactor Safety

Brookhaven National Laboratory

Upton, Long Is 1and, New York 11973

Hanford Engineering Development Laboratory

P. 0. Box 1970

Richland, WA 99352

Attn: L. D. Muhlestein

R. W. Wierman

R. K. Hilliard

G. R. Armstrong

R. P. Johnson

Atomics International Division

Engineering Department

8900 De Soto Avenue

Canoga Park, CA 91304

1000 G. A. Fow Ter

1100 C. D. Broyles

1120 G. E. Hansche

1126 G. L. Ogle

1130 H. E. Viney

1136 J. H. Davis

1136 J. A. Brammer

1136 H. C. Walling

1200 W. A. Gardner

1260 K. J. Touryan

1261 D. F. Mcley

1262 H. C. Hardee

1350 R. G. Clem (2)

2151 F. R. Kroeger

2540 0. M. Stuetzer

2547 G. W. Gobeli

2541 K. T. Stalker

3284 R. P. Tyler

5000 A. Narath
5100 J. K. Galt

5110 F. L. Vook

5120 G. J. Simmons

5130 G. A. Samara

5150 J. E. Schirber

5160 W. Herrmann

5167 B. M. Butcher

5167 J. E. Smaardyk

5167 H. J. Sutherland

5231 J. H. Renken

5231 J. A. Halbleib

5237 P. McDaniels

5400 A. W. Snyder (2)

5410 D. J. McCloskey

5411 D. A. Dahigren

5411 P. W. Conrad

5411 A. S. Benjamin

5411 R. L. Knight

5412 L. D. Buxton

5412 J. F. Muir

5412 W. B. Murfin

5420

5422

5422

5422

5422

5422

5423

5423

5423

5423

5423

5423

5423

5423

5423

5425

5425

5425

5425

5425

5430

5431

5431

5440

5443

5450

5451

5452

5452

5452

5800

5820

5822

5830

5831

5831

J. Walker

R. L. Coats

H. G. Plein

K. 0. Reil

J. B. Rivard

D. J. Sasmor

J. E. Powell

L. M. Choate

L. R. Edwards

J. G. Kelly

D. A. McArthur

A. R. Phillips

H. L. Scott

W. H. Sullivan

S. A. Wright

W. J. Camp

D. H. Nguyen

R. W. Ostensen

D. C. Williams

M. F. Young

R. M. Jefferson

R. M. Jefferson (Actg.)

C. M. Stone

R. W. Lynch

B. D. Zak

J. A. Reuscher

T. R. Schmidt

L. D. Posey

P. S. Pickard

J. T. Hitchcock

R. S. Claassen

R. L. Schwo il

R. W. Wh

M. '

$\mathrm{N}$

(10) 


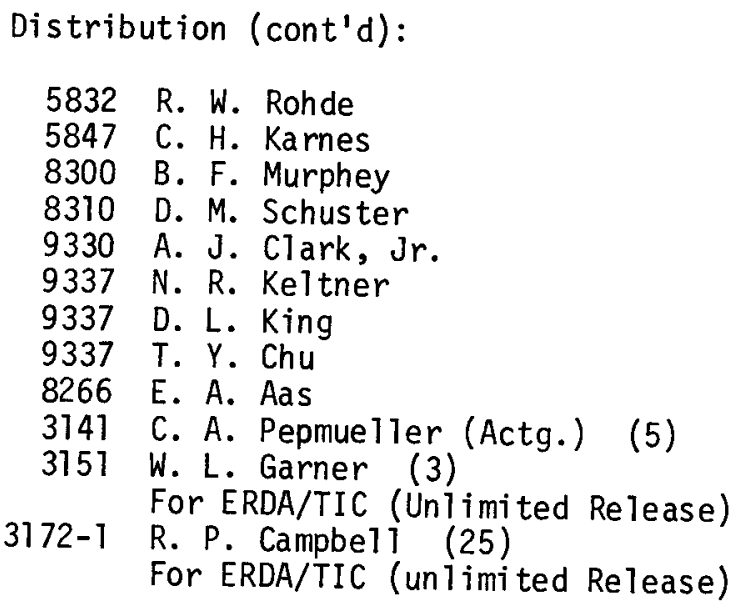

